\title{
Molecular docking studies of coumarin hybrids as potential acetylcholinesterase, butyrylcholinesterase, monoamine oxidase A/B and $\beta$-amyloid inhibitors for Alzheimer's disease
}

\author{
Samina Khan Yusufzai ${ }^{1}$, Mohammad Shaheen Khan ${ }^{2 *}$, Othman Sulaiman ${ }^{1}$, Hasnah Osman ${ }^{3}$ \\ and Dalily Nabilah Lamjin²
}

\begin{abstract}
Coumarins are the phytochemicals, which belong to the family of benzopyrone, that display interesting pharmacological properties. Several natural, synthetic and semisynthetic coumarin derivatives have been discovered in decades for their applicability as lead structures as drugs. Coumarin based conjugates have been described as potential AChE, BuChE, MAO and $\beta$-amyloid inhibitors. Therefore, the objective of this review is to focus on the construction of these pharmacologically important coumarin analogues with anti-Alzheimer's activities, highlight their docking studies and structure-activity relationships based on their substitution pattern with respect to the selected positions on the chromen ring by emphasising on the research reports conducted in between year 1968 to 2017.
\end{abstract}

Keywords: Coumarin, Neurodegenerative disorder, Alzheimer's disease, Acetylcholinesterase, Butyrylcholinesterase, Monoamine oxidase

\begin{abstract}
Introduction
Alzheimer's disease (AD) is the most common form of neurodegenerative disorder and the most prevalent cause of dementia commonly affecting the elderly. It is a progressive disorder of the brain that is associated with the loss of presynaptic markers of the cholinergic system in the brain, which is related to memory and ability to carry out daily activities. It is said to be progressive as its symptoms worsen over time. Two main causes of $\mathrm{AD}$ are plaques and neurofibrillary tangles (NFTs) which results due to the accumulation of beta-amyloid protein $(A \beta)$ outside the neurons. $A \beta$ is formed by the proteolytic cleavage of amyloid precursor protein (APP) which occurs by $\alpha$-secretase and is aberrantly processed by $\beta$ - and $\gamma$ - secretases resulting in an imbalance between
\end{abstract}

\footnotetext{
*Correspondence: shaheenchem@gmail.com

${ }^{2}$ Industrial Chemistry Programme, Faculty of Science and Natural Resources, Universiti Malaysia Sabah, 88400 Kota Kinabalu, Sabah, Malaysia

Full list of author information is available at the end of the article
}

production and clearance of $A \beta$ peptide and thus $A \beta$ forms highly insoluble and proteolysis resistant fibrils known as senile plaques (Fig. 1) [1]. These plaques will interrupt the neuron transmission at synapses and cause information transfer to fail leading to the neuronal cell death. NFTs are composed of the tau amyloid protein fibrils (Fig. 2) [2].

Tau is a component of microtubules that provides the internal support structure for the transport of nutrients and essential molecules within the cell. When tau is hyperphosphorylated, it forms insoluble fibrils that blocks the transport of nutrients and essential molecules in the neuron thus leading to cell death [3]. Jack et al. proposed a hypothetical model which explains about the progression of $\mathrm{AD}$ and how pathological events such as deposition of $A \beta$ fibrils and increased levels of tau protein in cerebrospinal fluid (CSF), lead to cognitive impairment and dementia (Fig. 3) [4]. To date, the cure of this disease is yet to discover. Nonetheless, researchers have found various alternatives to slow down its progression. Among these alternatives are, inhibition of acetylcholinesterase 

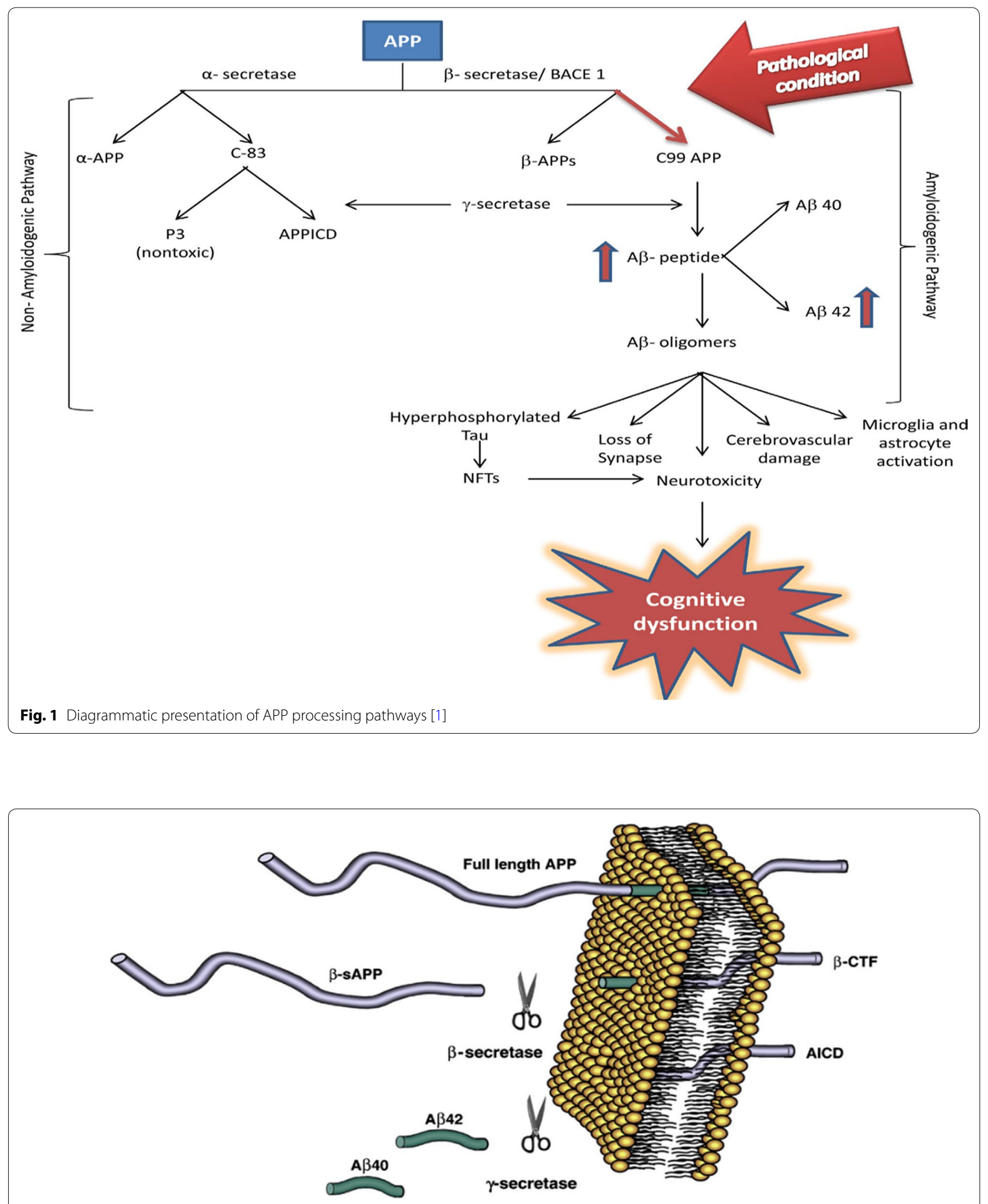

Fig. 2 Generation of soluble A fibrils from amyloid precursor protein [2] 


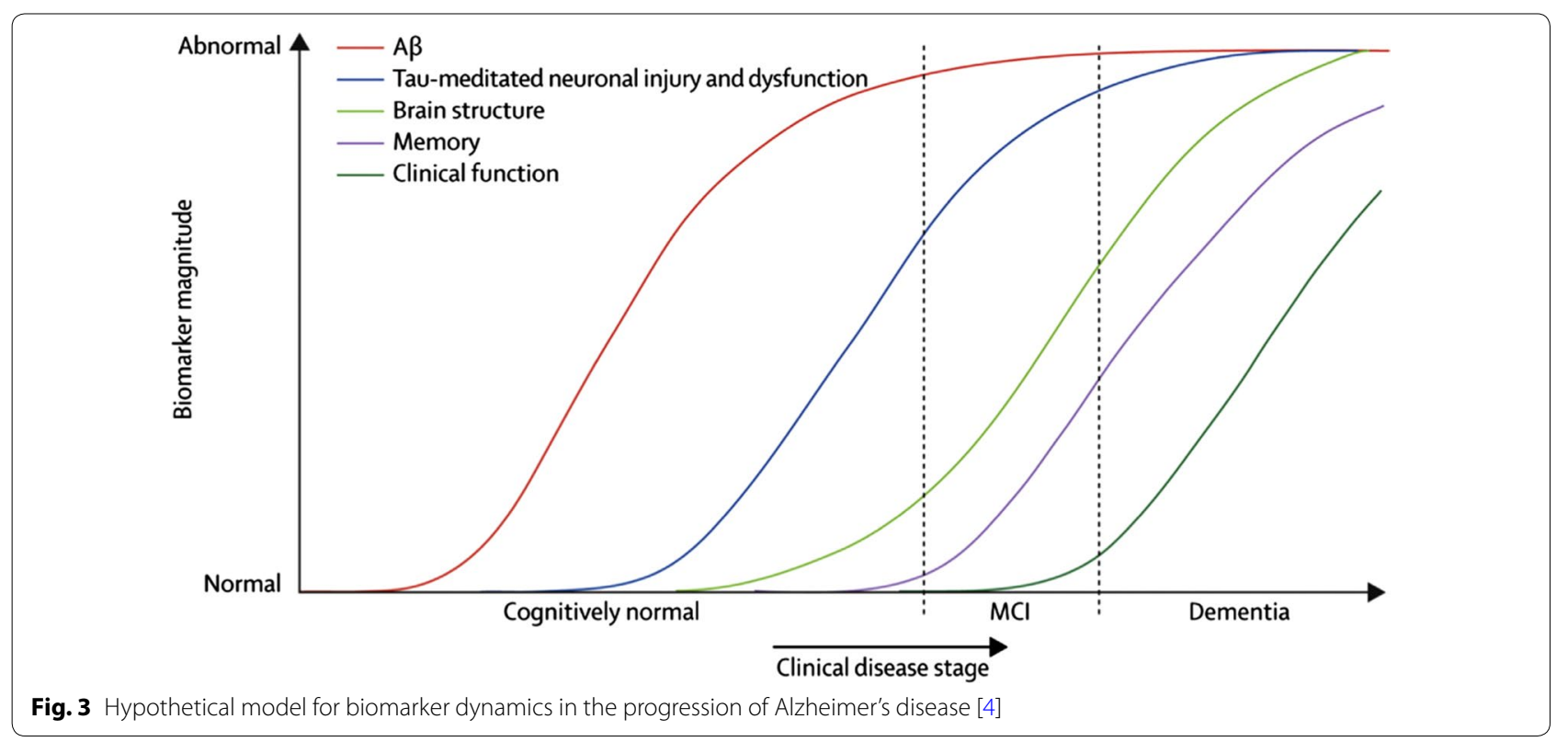

(AChE), APP, $\beta$-secretase, $\gamma$-secretase, monoamine oxidase (MAO) and metal chelators [5].

The first line treatment that is given to $\mathrm{AD}$ patients is AChE inhibitors because not only they facilitate cholinergic transmission, they also interfere with the synthesis, deposition and aggregation of toxic $\mathrm{A} \beta$. This might lead to the improvement of cognition and some behavioural problems [6-8]. The enzyme butyrylcholinesterase $(\mathrm{BuChE})$ has the same role as $\mathrm{AChE}$, which is to hydrolyse the acetylcholine in the synaptic cleft. However, their inhibition might help in enhancing the efficiency of treatment for the AD patients. Xie et al. stated that even though the activity of AChE decreases as the disease progresses, the activity of $\mathrm{BuChE}$ shows a significant increase in the hippocampus and temporal cortex. BuChE inhibitors might help to improve cholinergic activity by restoring the $\mathrm{AChE} / \mathrm{BuChE}$ activity ratios as seen in the healthy brain [9]. Recent investigations are focusing more on dual $\mathrm{AChE} / \mathrm{BuChE}$ inhibitors [8-10]. Monoamine oxidase $\mathrm{B}$ (MAO-B) is an important factor that is involved in oxidative stress and oxidative stress is said to be among the multiple factors, which induce the $\mathrm{AD}$. It is widely established in the literature that the activity of MAO-B can increase up to threefold in the temporal, parietal and frontal cortex of AD patients as compared to the controls. This increase in MAO-B activity produces higher levels of $\mathrm{H}_{2} \mathrm{O}_{2}$ and oxidative free radicals, which has been correlated, with the development of $\mathrm{A} \beta$ plaques. MAO-B inhibitors, hold the potential to be developed into effective anti-Alzheimer's drugs, as it has been reported before, that MAO-B inhibitors such as selegiline and rasagiline has shown to significantly improve the learning and memory deficits in the animal models, associated with $\mathrm{AD}$ and to slow the disease progression in $\mathrm{AD}$ patients [11-13]. Zatta et al. reported that dyshomeostasis and miscompartmentalization of metal ions such as $\mathrm{Fe}^{2+}, \mathrm{Cu}^{2+}$ and $\mathrm{Zn}^{2+}$ occurs in the brain of $\mathrm{AD}$ patients. The formation of $\mathrm{A} \beta$ plaques, neurofibrillary tangle as well as production of reactive oxygen species (ROS) and oxidative stress are closely linked to the highly concentrated metal ions in the neuropil and plaques of the brain [14]. Modulation of such biometals in the brain represents an additional rational approach for the treatment of $\mathrm{AD}[12,14]$. Another approach that gained interest among the researchers was to lower the $A \beta$ level by inhibiting the $\beta$-secretase (BACE1), which is a transmembrane aspartyl protease, responsible for $\mathrm{N}$-terminal cleavage of the APP which leads to the production of $\mathrm{A} \beta$ peptide [15].

Coumarin and its derivatives are reported to display wide range of biological activities such as anti-diabetic and antidepressant [16], anti-oxidant [17], anti-cancer [18], anti-proliferative [19], antinociceptive [20], antibacterial and anti-tubercular [21], hepatoprotective, anti-allergic, anti-HIV-1, antiviral, antifungal, antimicrobial and antiasthmatic [22]. The benzopyrone moiety of the coumarin nucleus is known as the fundamental for the design of hybrid molecule that can simultaneously inhibit AChE and AChE induced $\beta$-amyloid accumulation. Studies have also shown that naturally occurring as well synthetic coumarin analogues exhibit potent AChE, $\mathrm{BuChE}$, dual AChE/BuChE and MAO inhibitory activity [12, 23-25]. Coumarin's versatility allows chemical substitutions to occur at different sites in its structure, thus 
<smiles>O=c1oc2c3c4c(cc2c2c1CCC2)CCCN4CCC3</smiles>

Fig. 4 Molecular structure of coumarin 106 or C1

making it a compelling molecule for drug discovery [7, 8]. Modification of coumarin ring to develop new analogues of coumarin with superior activity is the main focus of the current review which is based on the reports which were taken in between the year 1968-2017.

\section{Coumarin analogues as $\mathrm{AChE}$ inhibitors}

Fallarero et al. [7] reported an active AChE inhibitor among a coumarin library consisting of 29 coumarins, including coumarin itself and several derivatives of the 7-hydroxy and 7-methoxy-coumarin as well as seven synthetic coumarins. The molecule that showed most active AChE inhibitory activity is $\mathbf{C 1}$, chemically known as $2,3,5,6,7,9,10,11$-octahydrocyclopenta[4,5]pyrano[2,3f]pyrido[3,2,1-ij]quinolin-12(1H)-one or coumarin 106 (Fig. 4).

In order to recognize how this compound act as AChE inhibitor and interacts with the target, Fallarero et al. [7] docked C1 into the enzyme AChE and predicted its binding mode. The result that was obtained showed that $\mathbf{C 1}$ was able to penetrate into the enzyme's active site gorge and bind to the AChE peripheral anionic site (PAS) as a secondary binding site (Fig. 5). It was reported before that binding to the PAS of the AChE might decrease the accumulating effects of the enzyme on the $\beta$-amyloid peptide, and hence the ability of $\mathbf{C 1}$ to bind to the peripheral anionic site of AChE proves its potential as drug lead or molecular probe for the $\mathrm{AD}$ treatment [7].

Razavi et al. designed and synthesized a series of 4-hydroxycoumarin derivatives [27]. They screened them towards electrophorus electricus acetylcholinesterase (eelAChE) and horse serum butyrylcholinesterase (eqBuChE) using modified Ellman's methodology, which was previously described by Kapkova et al. [28]. Commercially available donepezil, was used as the internal standard. Donepezil is one of most used AChEIs in AD therapy, acting as a dual binding site, reversible inhibitor of AChE with high selectivity over $\mathrm{BuChE}$. The result obtained showed that among the 19 coumarin derivatives, the acetamide pendent (C2) derivative, $N$-(1-benzylpiperidin-4-yl)acetamide (C3) (Fig. 6), displayed the highest AChE inhibitory activity with the $\mathrm{IC}_{50}$ value of $1.2 \mu \mathrm{M}$. The increase in

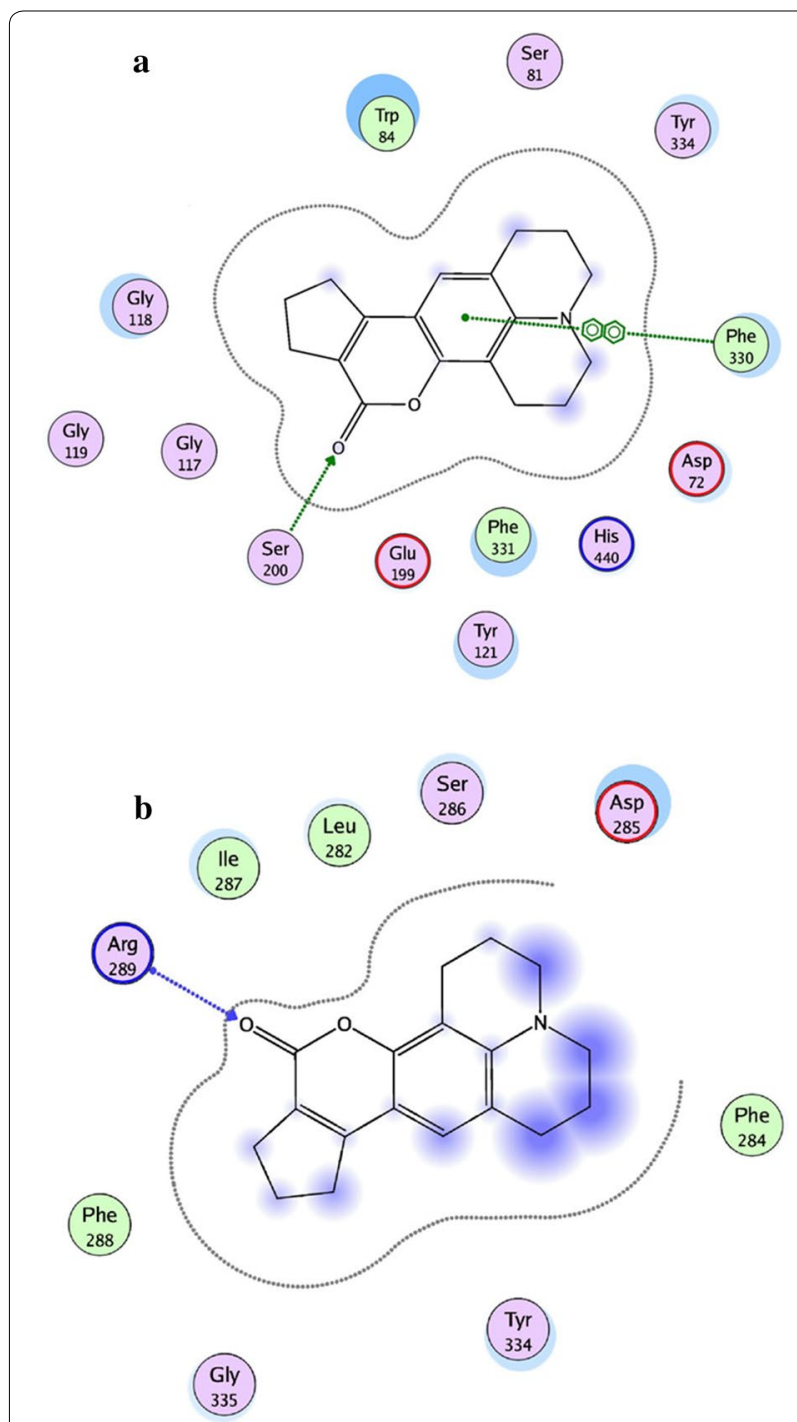

Fig. $\mathbf{5}$ a Propose binding of $\mathbf{C} \mathbf{1}$ at the active gorge site. $\mathbf{b}$ Propose binding of $\mathbf{C} \mathbf{1}$ at the peripheral anionic site [7]

this activity was further justified by the help of docking study of C3. The best docking pose of $\mathbf{C 3}$ and amino acids in the active site of Torpedo californica acetylcholinesterase (TcAChE) is represented in Fig. 7.

It was stated, that the type of cyclic amine attached to the 2-oxo or 4-oxoakoxycoumarin backbone influenced the increase in the inhibitory property. The strong antiAChE activity of $\mathbf{C} \mathbf{3}$ was found to be due to the ligand recognition and trafficking, for which Phe330 was responsible, through the formation of a $\pi$-cation interaction with the ligand, at the bottom of the active site of TcAchE. Additionally, the $\pi-\pi$ interaction between the coumarin moiety and Trp279 of PAS was also found to stabilize the ligand in the active site of TcAchE, due to which the enzyme inhibition was more potent. 


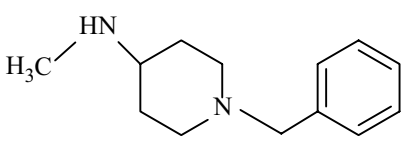

Fig. 6 Acetamide pendent derivative $\mathbf{C} 3$

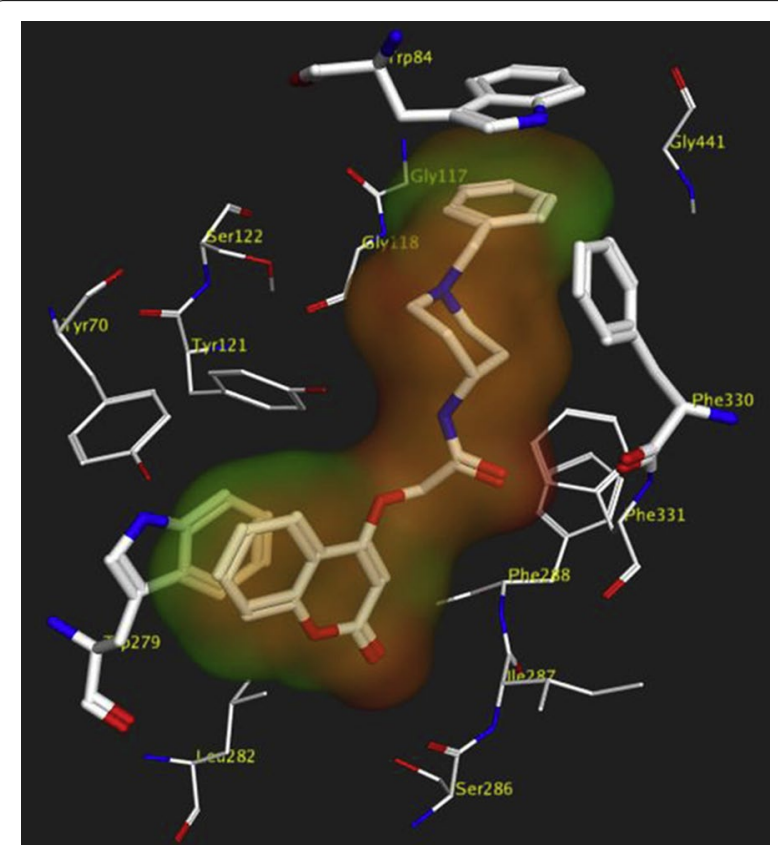

Fig. 7 Proposed binding mode of compound $\mathbf{C} 3$ within the active site of TCAChE [27]

Nam et al. [29] synthesized a series of aminoalkyl coumarin hybrids based on the structure of scopoletin and tested their in vitro $\mathrm{AChE}$ inhibition properties using mouse brain homogenates the internal standards scopoletin (C4) and galantamine (C5) (Fig. 8). It was reported that among all the derivatives synthesized, the pyrrolidine-substituted coumarins 7-Hydroxy-6-(2-(pyrrolidin1-yl)ethoxy)-2H-chromen-2-one hydrochloride (C6) and
7-Hydroxy-6-(3-(pyrrolidin-1-yl)propoxy)-2H-chromen2-one hydrochloride $(\mathbf{C} 7)$, exhibited the most potent inhibitory activities with $\mathrm{IC}_{50}$ values of 6.85 and $2.87 \mu \mathrm{M}$ and compound $\mathrm{C} 7$ was even found to express a 160-fold higher anti-AChE property than the lead structure scopoletin $\left(\mathrm{IC}_{50}=476.37 \mu \mathrm{M}\right)$ and nearly equal to that of galantamine $\left(\mathrm{IC}_{50}=2.50 \mu \mathrm{M}\right)$. Additionally, these derivatives also ameliorated scopolamine-induced memory deficit in mice when they were fed orally at the dose level of 1 and $2 \mathrm{mg} / \mathrm{kg}$. The activity profiles of $\mathbf{C 6}$ and $\mathbf{C 7}$ are shown in Fig. 9 [29].

Singla and Piplani synthesized a series of 15 novel coumarin hybrids in which coumarin moiety was linked to different substituted amines via an appropriate linker as potential inhibitors of AChE [30]. They performed the molecular docking studies in order to evaluate their potential as dual binding site acetylcholinesterase inhibitors for the treatment of cognitive dysfunction caused by increased hydrolysis of acetylcholine and scopolamine induced oxidative stress. Among all the synthesized compounds, the compound 4-[3-(4-phenylpiperazin-1-yl) propoxy]-2Hchromen-2-one (C8), was found to be post potent displaying higher $\mathrm{AChE}$ inhibitory activity of $\mathrm{IC}_{50}=2.42 \mu \mathrm{M}$ against the standard drug donepezil with the $\mathrm{IC}_{50}$ value of $1.82 \mu \mathrm{M}$ and hence displaying significant binding interactions with both the binding pockets viz Trp86 and Trp286, respectively, of the acetylcholinesterase enzyme (Fig. 10). Molecular docking study of C8 indicated that it interacts with all the crucial amino acids present at the catalytic active site (CAS), mid-gorge and PAS of TcAChE through hydrophobic, Van der Waal and $\pi-\pi$ stacking interactions resulting in higher inhibitory potency of AChE enzyme as compared to other 14 analogues of the series.

In detailed observation it was reported that the phenylpiperazine fragment of compound $\mathbf{C 8}$ was found to enter into the gorge of the $\mathrm{AChE}$ enzyme, resulting in parallel $\pi-\pi$ stacking interactions with the catalytic site of amino acids Trp86 (4.32 $\AA$ ) and His447 (4.97 $⿱$ A), thus adopting a sandwich like form. Coumarin moiety was also found to<smiles>O=c1ccc2cc(OCCN3CCCC3)c(O)cc2o1</smiles><smiles>O=c1ccc2cc(OCCCN3CCCC3)c(O)cc2o1</smiles>

Fig. 8 Standards scopoletin (C4) and galantamine (C5) 


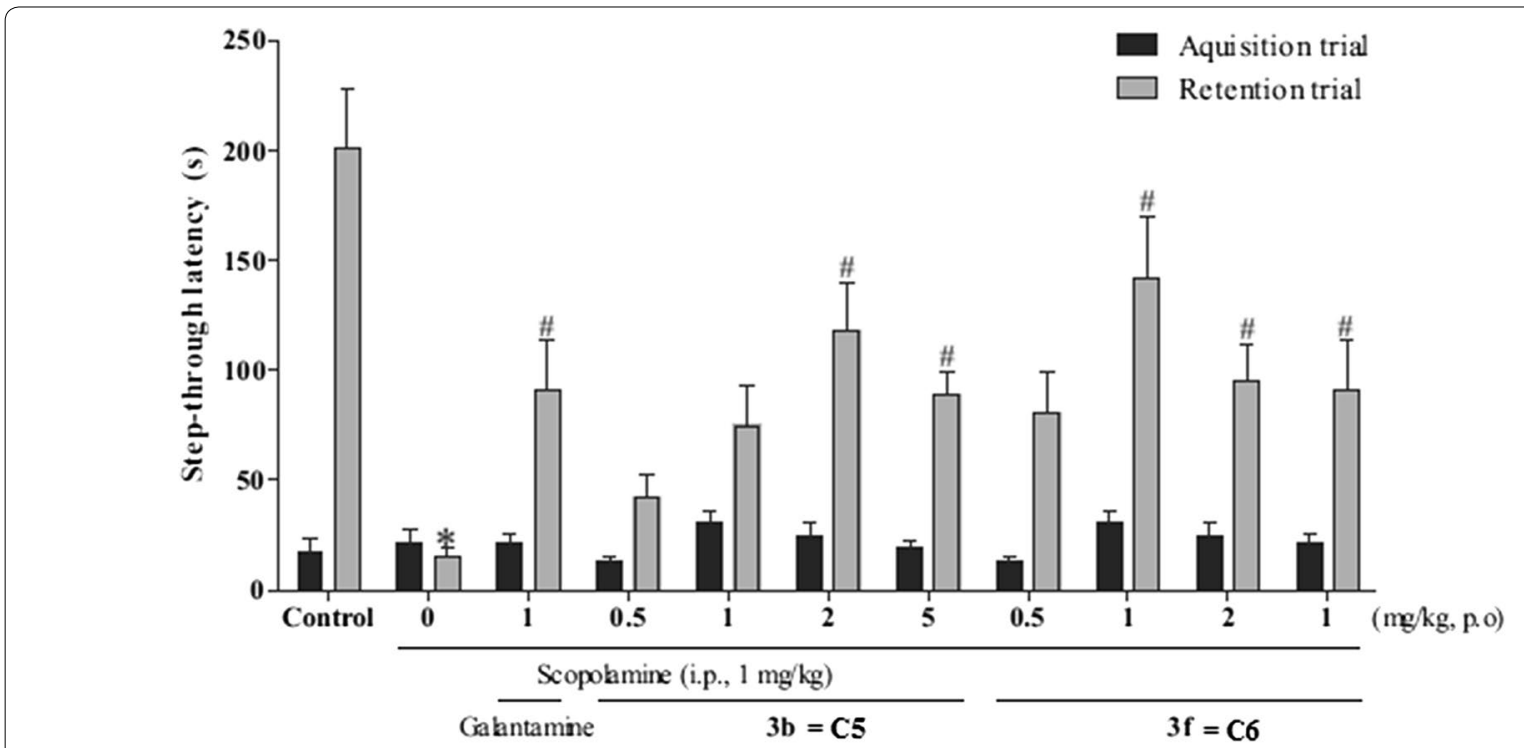

Fig. 9 Effects of $\mathbf{C 6}, \mathbf{C 7}$, and galantamine on the passive avoidance task in scopolamine-induced memory deficit model. *P $<0.05$ versus vehicle-treated controls. ${ }^{P} P<0.05$ versus scopolamine-treated group. Data are expressed as mean \pm SEM [29]

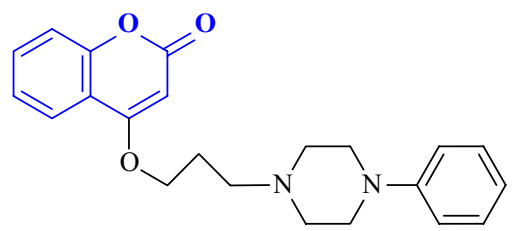

Fig. 10 Phenylpiperazine derivative $\mathbf{c} 8$ interact via aromatic $\pi-\pi$ interactions with ring-to-ring distance of 4.5-4.7 $\AA$ with the indole and phenyl rings of Trp286 and Tyr72, which were located at the peripheral anionic site (Fig. 11).

Zhou et al. designed and synthesized three series of coumarin derivatives (Series A, B and C) with different phenylpiperazine moiety as substituents to study their
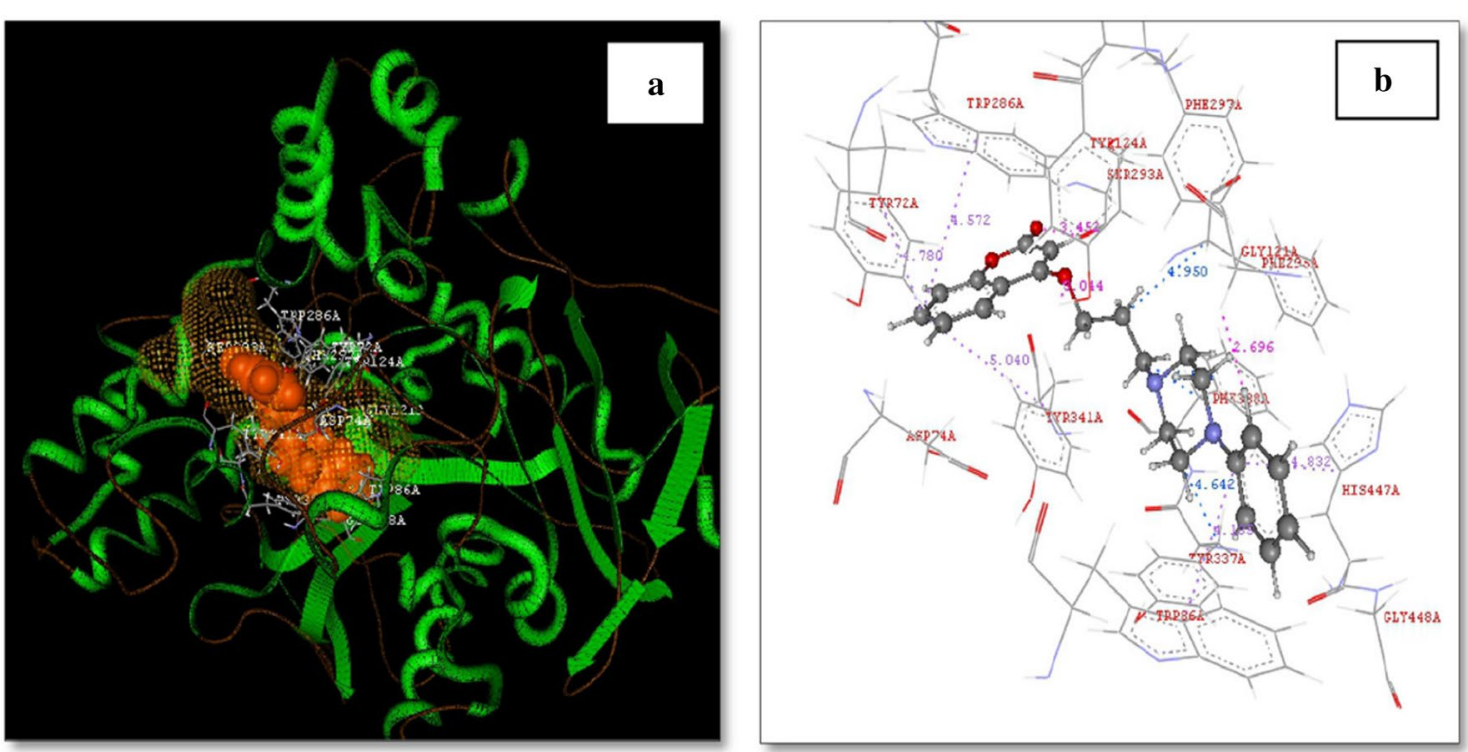

Fig. 11 a 3D Orientation of the best docked pose of compound $\mathbf{C} 8$ in the active site cavity of AChE. $\mathbf{b}$ Hypothetical binding motif of $\mathbf{C} 8$ within the crystal structure of AChE surrounding active amino acids [30] 
potential for the treatment of AD with respect to donepezil, a standard drug [31]. While designing their series they minutely considered quite a number of factors, which influence or affect the inhibition of cholinesterase enzyme. As recent research have revealed that those compounds which can effectively dual-bind with AChE possess very good therapeutic importance as they can effectively cause the prevention of $A \beta$ aggregation. These therapeutic acetylcholinesterase inhibitors (AChEIs) can facilitate cholinergic transmission, interfere with the synthesis, deposition and aggregation of toxic $A \beta$-peptides $[32,33]$. Certain anti-AChEIs, which play effective role in the memory improvement and cognitive functions and are used to treat AD on clinical level for past many years are reportedly: tacrine, donepezil, rivastigmine, and ensaculin (Fig. 12). Among all these standard drugs specifically ensaculin which is a derivative of coumarin and composed of benzopyran and piperazine moiety has been used in its $\mathrm{HCl}$ salt form the under trade name of KA-672 $\mathrm{HCl}$ has been reported to slow down or prevent this progressive neurodegeneration [34, 35].

The Torpedo California has reported the three dimensional X-ray structure of AChE for comparative study between the enzyme and the inhibitors [36] and the X-ray structure of the transition state of the AChE has also been reported [37]. Figure 13 shows the insight structure of the active sites of AChE which mainly consists of 4 binding sites: (i) Anionic substrate (AS) binding site-contains Trp84, Glu199, and Phe330 aromatic residues with negative charges where the nitrogen of quaternary ammonium group of $\mathrm{AChE}$ and various other positive active sites bind through interaction between the quaternary nitrogen (or other active sites) and electrons of the aromatic residues. (ii) Ecstatic site (ES): contains three residues Ser200-His440-Glu32718 which forms the catalytic triad. (iii) Acyl binding site (ABS): consists of Phe288, and Phe299, which binds to

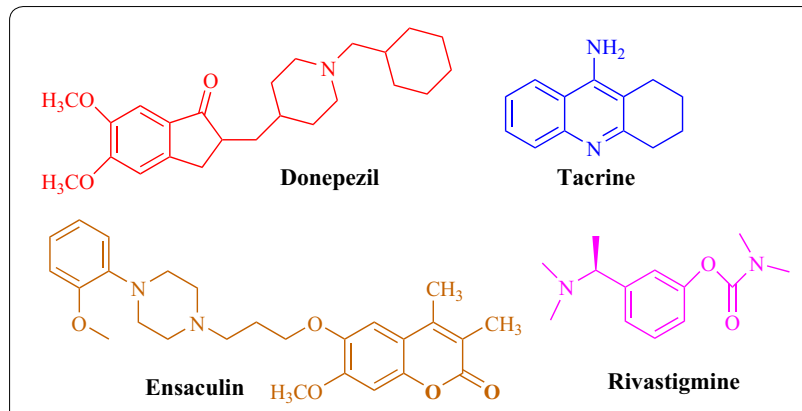

Fig. 12 Structures of the acetylcholinesterase inhibitors as FDA approved Alzheimer's disease therapeutics

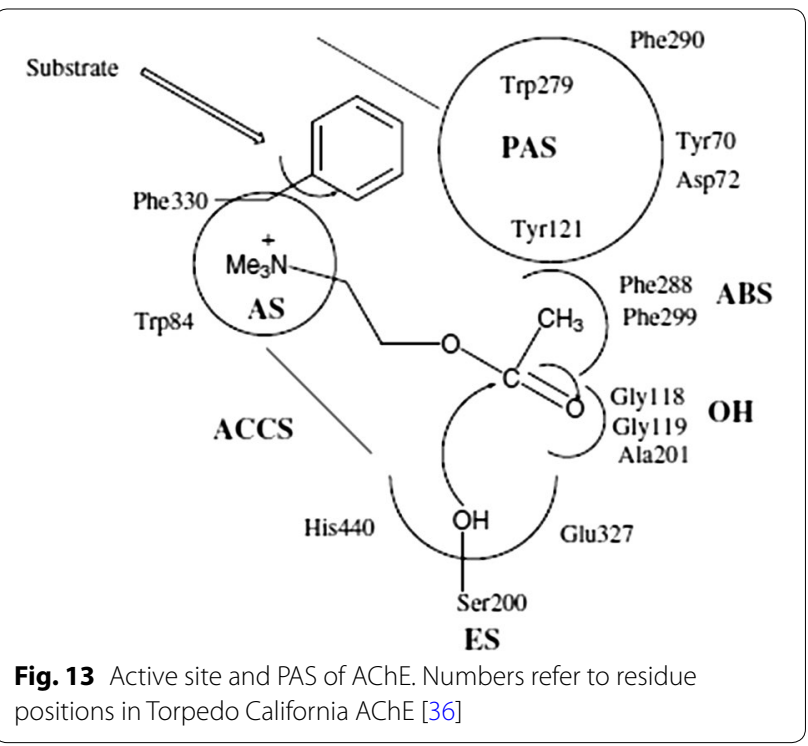

the acetyl group of AChE enzyme [33]. (iv) PAS: consists of Trp279, Tyr70, Tyr121, Asp72, Glu199, and Phe290, which can bind to 9-aminoacridine (tacrine) [38-41]. Exclusively molecules are shown to interact with PAS but can also interact with both viz catalytic ES and PAS and this helps in the prevention of proaggregation of $A C h E$ toward $A \beta$ [42].

These factors were the core reason behind choosing ensaculin, as the basic skeleton and designing the three series of anti-AChE coumarins analogues with phenylpiperazine substituted at position 6 of coumarin in series A, at position 3 in series $B$ and at position 4 in series $C$, which were similar in structure to the frame of ensaculin (Fig. 14). Donepezil was chosen as the standard drug for comparing the obtained results.

The anti-AChE results concluded that coumarin derivatives with substitution at position 3 (series B) and 4 (series $C$ ) of the coumarin ring were better that the derivatives with substitution at position 6 (series A). On comparison of series A with ensaculin, the reason behind the dullness was clear. It was concluded that the presence of only one atom in the linking chain between coumarin skeleton and phenylpiperazine moiety in series A was the cause for this as it cannot reach the requirement for gorge, with respect to the presence of four atoms in ensaculin [33]. The two most potent 4-phenylpiperazine substituted coumarin derivatives of series $\mathrm{C}$ were 6-methyl-4-(4-phenylpiperazin-1-yl)2Hchromen-2-one (C9) and 6-methyl-4-(4-(4-methylbenzoyl)piperazin-1-yl)2H-chromen-2-one (C10) with $\mathrm{IC}_{50}$ value of 4.5 and $5.3 \mu \mathrm{mol} / \mathrm{L}$. The distance between carbonyl-carbon atom and nitrogen atom of piperazine 


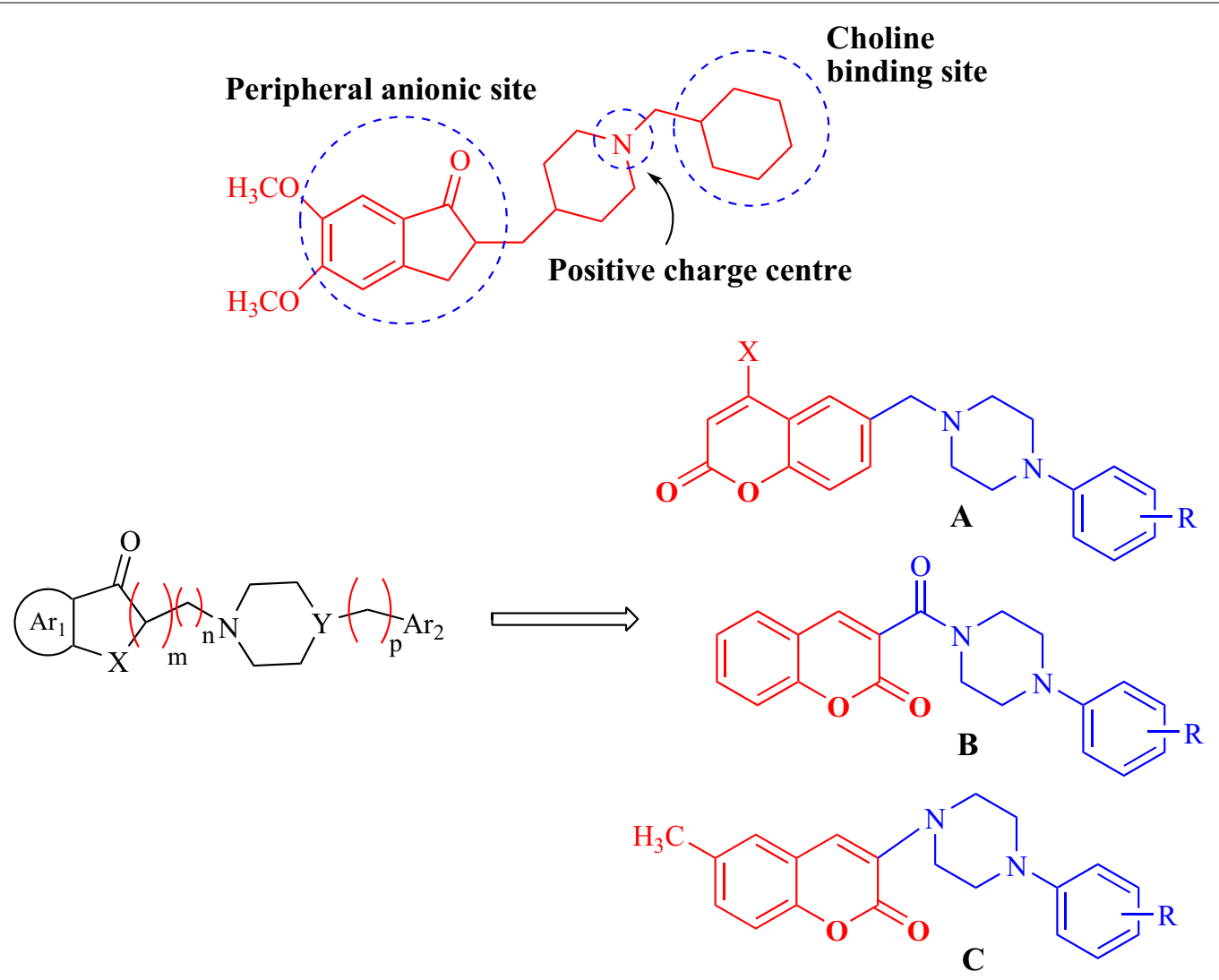

Fig. 14 Design strategy of the target compounds of series A, B and C

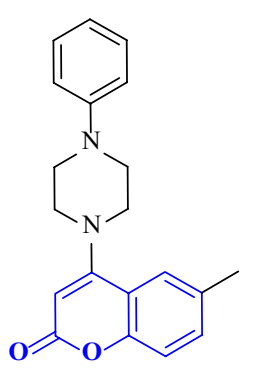

C9

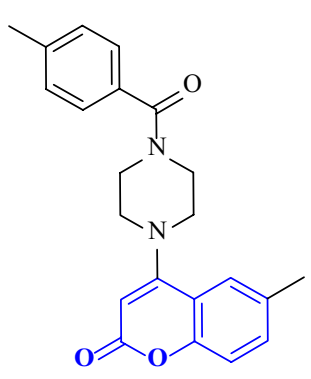

C10
Fig. 15 4-Phenylpiperazine substituted coumarin derivatives $\mathbf{C}$ and C10

ring was in agreement with donepezil hence making these 4-substituted compounds good anti-AChE inhibitors (Fig. 15).

\section{Coumarin analogues as dual $\mathrm{AChE} / \mathrm{BuChE}$ inhibitors}

Khoobi et al. have designed and synthesized novel hybrid derivatives of 2 known scaffolds which are tetrahydroaminoquinoline and coumarin to form novel series of 8-amino-tetrahydrochromeno[3', $\left.4^{\prime}: 5,6\right]$ pyrano[2,3-b] quinolin-6(7H)-one. These derivatives were screened for their $\mathrm{AChE} / \mathrm{BuChE}$ inhibitor activities using colorimetric Ellman's method. The result obtained showed that compound 8-amino-7-(4-fluorophenyl)-9,10,11,12-tetrahydrochromeno $\left[3^{\prime}, 4^{\prime}: 5,6\right]$ pyrano[2,3-b]quinolin-6(7H)-one (C11) was the most active compound against eelAChE whereas compound 8-amino-7-(benzo[d][1,3]dioxo5-yl)-9,10,11,12-tetrahydrochromeno $\quad\left[3^{\prime}, 4^{\prime}: 5,6\right]$ pyrano [2,3-b]quinolone-6(7H)-one $(\mathbf{C 1 2})$ showed the most potency as BuChE inhibitor (Fig. 16) [8].

Khoobi et al. stated that the increase in the inhibitory activity of compound (C11) might have been caused by the insertion of aromatic groups at position 7 , and the 
<smiles>Nc1c2c(nc3c1C(c1ccc(F)cc1)c1c(c4ccccc4oc1=O)O3)CCCC2</smiles>

C11<smiles>Nc1c2c(nc3c1C(c1ccc4c(c1)OCO4)c1c(c4ccccc4oc1=O)O3)CCCC2</smiles>

C12
Fig. 16 Molecular structure for C11 and C12

presence of electron withdrawing substituents that is fluoro at position 4 , that was able to form $\pi-\pi$ interaction in the hydrophobic cavity. In addition, the increased activity of (C12) was due to the lipophilic interaction of electron donating groups such as methyl and methoxy with the active site. They carried out a kinetic study on compound $\mathbf{C 1 1}$ to determine the kinetic type of AChE inhibition and the result showed that it possessed a mixed type inhibition where it can interact with both, PAS and CAS. To identify the binding mode of compound C11, it was docked at the gorge of eelAChE. The obtained result showed a proper fitting of compound C11 in the gorge of eelAChE (Fig. 17). The phenyl ring at position 7 was turned towards the hydrophobic pocket of the binding cavity composed of Phe330, Tyr334 and Phe331, and the ligand-receptor complex was stabilized by the $\pi-\pi$ stacking between the phenyl side chain of Tyr334 and the phenyl moiety of compound C11. $\pi-\pi$ stacking between pyridine ring and indole side chain of Trp279 was able to donate specific conformation to the compounds so that the lipophilic cyclohexane ring gets fitted in the hydrophobic packet composed by Phe290, Leu282, Phe288, Ile287 and Ser286 whereas the coumarin carbonyl moiety forms a hydrogen bonding with hydroxyl of Tyr121 and $\mathrm{CH}-\pi$ stacking between side chain of Gln74 and phenyl ring of coumarin scaffold. Based on the results obtained, the hybrid of tetrahydroaminoquinoline and coumarin scaffold designed and synthesize by Khoobi et al. can undergo further modifications and proposes as promising lead structure.

Asadipour et al. designed novel coumarin-3-carboxamides bearing $N$-benzylpiperidine moiety as potent $\mathrm{AChE} / \mathrm{BuChE}$ inhibitors (Fig. 18). The docking result concluded that most of the synthesized hybrids were potent anti-AChE and among all the compounds, compound (C14a) displayed the most potent activity as
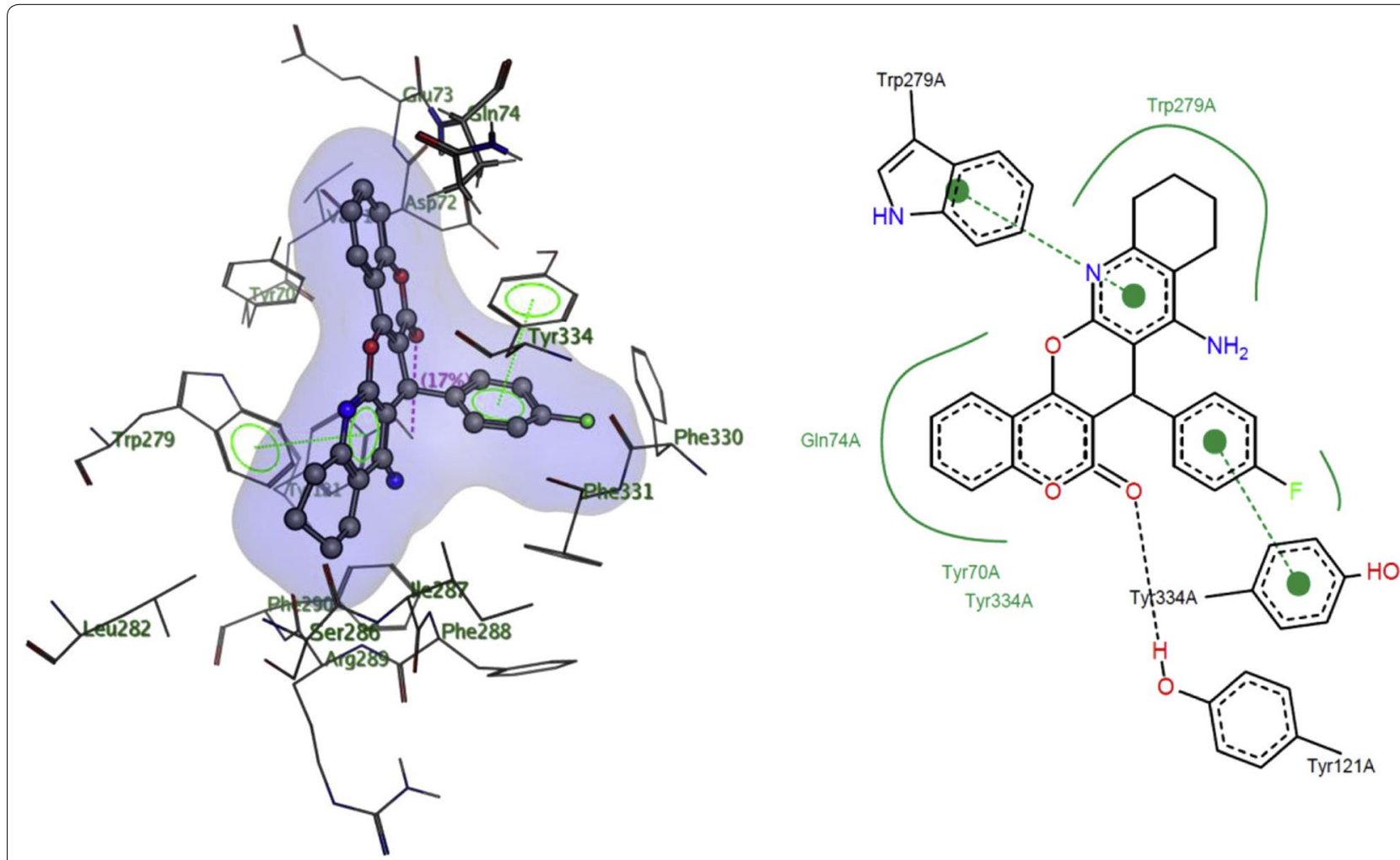

Fig. 17 Residues involved in the interactions with $\mathbf{C 1 1}$ and the 2D representation of binding mode of $\mathbf{C 1 1}$ in the gorge of AChE [8] 


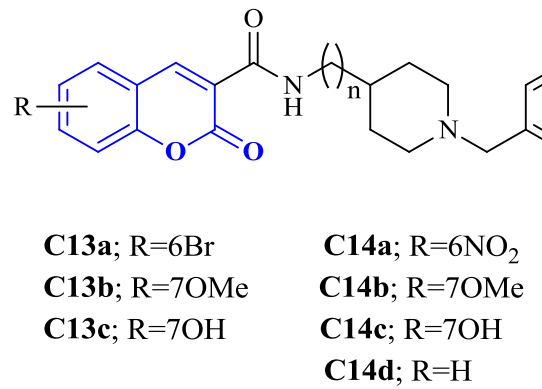

Fig. 18 Chemical structures of coumarin-3-carboxamides

anti-AChE inhibitor with $\mathrm{IC}_{50}$ of $0.3 \mathrm{nM}$, which was almost 46-fold more than the standard drug donepezil [10]. In depth structural study of protein-ligand demonstrated, that the synthesized compounds possessed dual binding site interaction mode, which was also in agreement with the performed kinetic studies. Recent studies have reported that dual inhibitory properties might help in improving the symptoms related with dementia and treating AD [43, 44]. This dual interaction mode of the compounds was due to its designing. The compounds were synthesized by linking two fragments, $N$-benzylpiperidine (CAS binding motif) and coumarin (PAS binding motif) with two types of linkers, carboxamide or $N$-ethylcarboxamide. It was reported that the compounds with $\mathrm{N}$-ethylcarboxamide linker were more active than their counterparts with carboxamide linker, which can be seen for compound C14a having a nitro group at position 6 of the coumarin ring. Presence of various substituent were found contributing for different level of anti-ChE activity. Bromo, nitro and methoxy substituents were reported to increase the anti-AChE activity whereas hydroxyl group at position 6 or 7 of the coumarin ring was found decreasing the AChE inhibitory activity. Comparison of 7-methoxy analogues $(\mathbf{C 1 3 b}$, carboxamide linked and C14b, $\mathrm{N}$-ethylcarboxamide linked) with the 7-hydroxy counterparts (C13c, carboxamide linked and C14c, $\mathrm{N}$-ethylcarboxamide linked) revealed, that O-methylation of 7-hydroxy coumarins improves the AChE activity. Compounds of $\mathrm{N}$-ethylcarboxamide series were also found to show higher BuChE inhibitory activity with the $\mathrm{IC}_{50}$ value $\leq 420 \mathrm{nM}$ as compared to the compounds of carboxamide series with the $\mathrm{IC}_{50}$ value of $\geq 20 \mu \mathrm{M}$. Additionally, it is quite interesting to find out that the position of methoxy group in 7-and 8-methoxy isomers, (linked through $\mathrm{N}$-ethylcarboxamide) was influencing BuChE/AChE selectivity in 71 versus 9000 ratio. However, $N$-ethylcarboxamide linked compound C14d, was reported as a dual cholinesterase inhibitor with more favorable balancing between $\mathrm{AChE} / \mathrm{BuChE}$ inhibitions as compared to the standard drug donepezil. It was found to display anti-AChE activity with $\mathrm{IC}_{50}$ $26 \mathrm{nM}$ and anti-BuChE activity with $\mathrm{IC}_{50} 371 \mathrm{nM}$.

In order to get the insight detail of the binding modes and structural modifications of the most active compounds from the carboxamide (C13a) and $\mathrm{N}$-ethylcarboxamide (C14a) linked series. Asadipour et al. performed automated docking against AChE using the optimized parameters. Both the active molecules were found to behave similarly in terms of orientation to that of the standard donepezil within the active site. This reveals the fact that the $N$-benzylpiperidine fragment of the compounds helped them to anchor in the midgorge of the enzyme and the coumarin part was accommodated in the rim of the gorge (Figs. 19 and 20). The good anti-ChE activity of the compounds of $\mathrm{N}$-ethylcarboxamide linker series over carboxamide linker series was found to due to their more favourable interactions with the targeted enzymes. The $\mathrm{N}$-ethylcarboxamide linked compound C14a was observed to form hydrogen bond in between the carbonyl of coumarin and hydroxyl of Tyr 121. Moreover, the carbonyl group of its amide moiety was also to be directed towards the hydrophobic pocket comprising of Ser286, Phe290, and Arg 289. Both these two interactions were not observed for the compound of carboxamide linker series (C13a). The common interactions, which were observed for both the linker compounds, were the $\pi-\pi$ interaction observed due to the parallel disposition of the phenyl ring of benzyl moiety to Trp84, a $\pi$-cation between the quaternary nitrogen of piperidine ring with Phe330 and a $\pi-\pi$ stacking of coumarin ring and Trp279.

Alipour et al. synthesized novel coumarin derivatives (Fig. 21) as potent and dual binding site acetylcholinesterase inhibitors bearing $N$-benzyl pyridinium moiety which was attached to the coumarin nucleus via alpha beta unsaturated carbonyl linker. The reason behind the introduction of this linker was its conformational restriction caused by the presence of conjugated double bond which makes the molecule free from any conformational alternations and helps in further study related to substituent modifications. Most of the designed compounds were found to exhibit $\mathrm{IC}_{50}$ values in nanomolar range and among all, compound (E)-4-(3-(6-Bromo-2-oxo2H-chromen-3-yl)-3-oxoprop-1-enyl)-1-(2-fluorobenzyl)pyridinium chloride (C15a) was found to be the most active against acetylcholinesterase enzyme displaying $\mathrm{IC}_{50}$ value of $0.11 \mathrm{nM}$ and compound (E)-1-(2Chlorobenzyl)-4-(3-(8-methoxy-2-oxo-2H-chromen-3yl)-3-oxoprop-1-enyl)pyridinium chloride $(\mathbf{C} 15 \mathbf{b})$ gave the most potent inhibition of $\mathrm{BuChE}\left(\mathrm{IC}_{50}=125 \mathrm{nM}\right)$. Alipour et al. observed that the steric and electronic features of the substituents in the coumarin nucleus played 

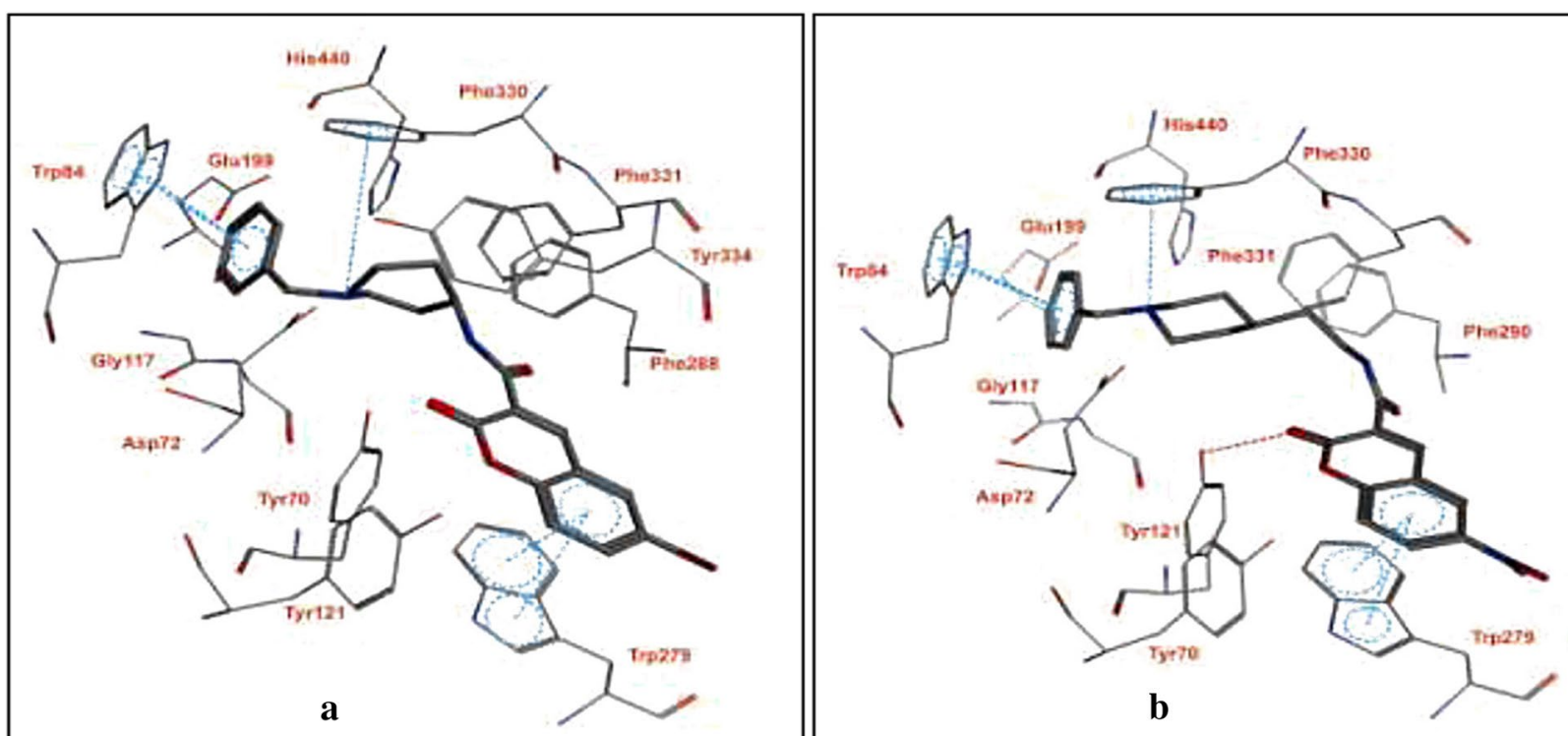

Fig. 19 a Representative model for interactions of compounds C13a. b C14a docked into the binding site of AChE. Hydrogen bond is indicated as red dotted line [10]

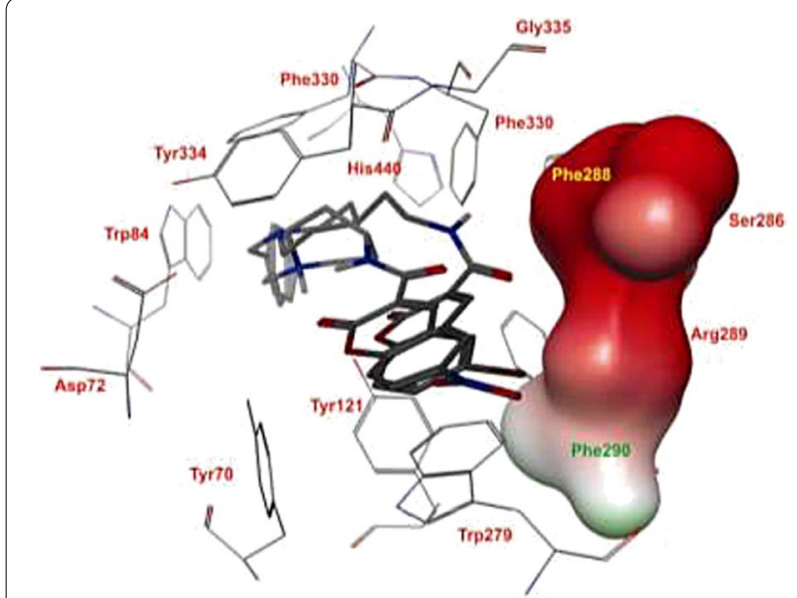

Fig. 20 The relative orientation of carboxamide linker of the best docking poses of $\mathbf{C 1 3 a}$ and $\mathbf{C 1 4 a}$. The carboxamide linker of $\mathbf{C 1 4 a}$ oriented to a hydrophillic pocket (red surface) of the active site [10]

a great role on the anticholinesterase activity of the target compounds. They reported that the movement of fluoro substituent from ortho-position (C15a) to para-position $\left(\right.$ C15c, $\left.\left(\mathrm{IC}_{50}=0.46 \mathrm{nM}\right)\right)$ causes a 4 times decrease in the inhibition properties.

These observations were also in agreement with the docking studies. Fluoro at ortho-position was found to disrupt the $\pi-\pi$ stacking interactions due to the rotation of the phenyl ring, which was in the other case observed for compound C15d. But the movement of fluoro to meta-position (C15e) increased the inhibitory properties due to proper stacking of the phenyl ring along with Trp84. Flouro at para-positionagain found to reduce the activity because of the steric hindrance with the amino acids present at the bottom of $\mathrm{AChE}$ gorge (Fig. 22). Additionally, larger sized substituents (C15b; $\mathrm{IC}_{50}=0.46 \mathrm{nM}$ ) were found to enhance the inhibitory activity regardless of their electronic properties as compared to the smaller sized substituents $(\mathbf{C 1 5 d}$; $\mathrm{IC}_{50}=26 \mathrm{nM}$ ). Moreover, the electron-withdrawing nature of the substituents present at position 3 of the benzyl moiety were found to enhance the activity according to their strong electron withdrawing nature $\mathrm{C15e}(\mathrm{F}$, $\left.\mathrm{IC}_{50}=0.47 \mathrm{nM}\right)>$ C15f $\left(\mathrm{CN}, \mathrm{IC}_{50}=76 \mathrm{nM}\right)>\mathbf{C 1 5 g}(\mathrm{Cl}$, $\mathrm{IC}_{50}=86 \mathrm{nM}$ ), whereas the insertion of any substituent at any position of the benzyl ring exceptionally decrease the inhibitory activities of the target compounds as observed for C15h $\left(\mathrm{IC}_{50}=330 \mathrm{nM}\right), \mathbf{C 1 5 i}\left(\mathrm{IC}_{50}=20 \mathrm{nM}\right)$ and C15j $\left(\mathrm{IC}_{50}=440 \mathrm{nM}\right)[45]$.

Khoobi et al. [8] designed and synthesized 5-oxo4,5-dihydropyrano[3,2-c]chromene derivatives and attached them to $N$-benzylpyridinium scaffold before subjecting them for their acetylcholinesterase (AChE) and butyrylcholinesterase (BuChE) inhibitory activities against the standard drug donepezil. From their findings, they found out that the 4-(2-Amino-3-cyano-5-oxo-4,5dihydropyrano[3,2-c]chromen-4-yl)-1-(4-fluorobenzyl) pyridinium chloride derivative (C16) was the strongest AChE inhibitor with $\mathrm{IC}_{50}$ value $0.038 \mu \mathrm{M}$ and strongest $\mathrm{AChE} / \mathrm{BuChE}$ selective with SI value of more than 


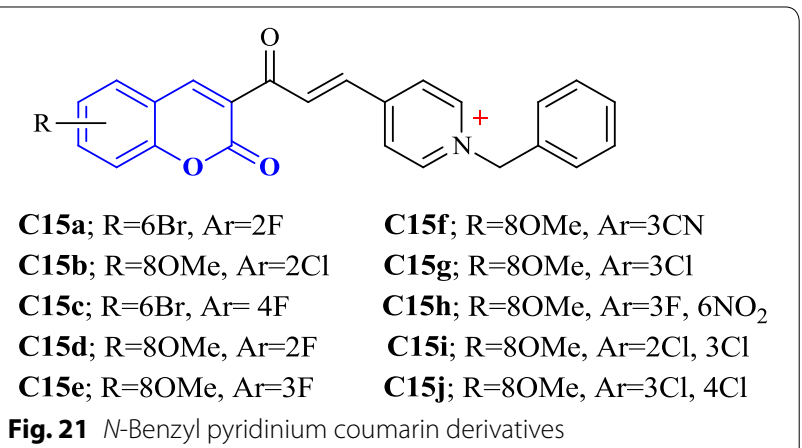

48 and compound 4-(2-Amino-3-cyano-5-oxo-4,5dihydropyrano[3,2-c]chromen-4-yl)-1-(2,4-dichlorobenzyl)pyridinium chloride (C17) was the strongest BuChE inhibitor with $\mathrm{IC}_{50}$ value of $0.566 \mu \mathrm{M}$ (Fig. 23).

To get the insight information related to structure activity relationship and possible binding modes they docked this compound into the active site of the enzyme using Autodock Vina 1.1.1. The ability of the target compounds to interact with the two distinct regions of the active site, which are the CAS and PAS is their most common feature. Within the active site of the enzyme AChE, the vicinity portion of the catalytic site (CS) is called as the central CAS and the top position of the gorge of $\mathrm{AChE}$ is called as PAS. The residues importantly responsible for the interaction with the target compounds (inhibitors) are Phe330, Trp84 from CAS to Trp279 from PAS to in all these Phe330 is the key residue for ligand recognition and trafficking. As the chemical space (inhibitor) is made up of two recognized fragments which are pyranochromen-2-one, and $N$-benzylpyridinium, therefore the pyrano chromene pendent of the inhibitor was found to oriented in such a manner that a $\pi-\pi$ interaction was achieved with Phe330 and Trp84 and the positively charged amino group on the pyran ring was prone to negative area by the Asp72 of carboxylic acid side chain. In addition to these a $\pi-\pi$ interaction between the benzyl group on pyridinium ring and Trp279 further stabilized the resulted complex (Fig. 24).

Same ways compound $\mathbf{C 1 7}$ was docked against BuChE and its mode of interaction is depicted in Fig. 25, where a $\pi-\pi$ stacking between the coumarin ring and Trp79 is quite clear. The orientation of the ligand (inhibitor/target compound) resulted in the exposure of the partially charged amino and nitrile groups to the oxyanionic hole composed of carbonyl moieties of Gln64, Asn65, Asp67, Asn80, Thr117 and Gly118. Additionally the hydrophobic moiety of the ligand i.e. 2,4-dichlorophenyl moiety was found to best fit in the hydrophobic pocket composed of Pro282, Leu283, Phe326, Tyr329 and Gly330 [46].
Alipour et al. [47] designed and synthesized the derivatives of 7-hydroxy coumarin and attached them to various amines via an amidic linkage, and further checked their potential as $\mathrm{AChE}$ and $\mathrm{BuChE}$ inhibitors using donepezil as a standard drug. Interestingly, benzylpiperidinylamino derivative, $N$-(1-benzylpiperidin-4-yl) acetamide (C18) was found to be the most potent compound against $\mathrm{AChE}$ displaying a very good $\mathrm{IC}_{50}$ value of $1.6 \mu \mathrm{M}$ and the (2-fluorophenyl)piperazine derivative (C19) was the most potent compound against BuChE with $\mathrm{IC}_{50} 15 \mu \mathrm{M}$. It is important to mention that the effect of structural modification was similar for both the activities but the most active compound against $\mathrm{AChE}$ was not the most active against BuChE. Therefore, further docking studies were performed in order to get the clue behind this interesting behaviour of C18 (Fig. 26).

Autodoc Vina program was used to examine the bestdocked pose of all the synthesized inhibitors. It was found that all the inhibitors were oriented similarly into the active site of the enzyme. However, for the most active compound $\mathbf{C 1 8}$, interesting results were noted down (Fig. 27). The ligand was found to be nicely accommodated in the gorge of AChE active site, in such a manner that the benzylpiperidin moiety was noted to lean towards CAS. A T-shape edge-to-edge $\pi-\pi$ stacking interaction of phenyl ring against Trp83 was observed, specifically and piperidine ring at the vicinity of Phe329 and phe330 achieved a $\pi$-cation interaction. Nevertheless, coumarin ring was noted to gain a $\pi$-stacking with the aromatic ring of Trp278 in PAS and its carbonyl moiety was found bonded to Arg288 via hydrogen bond. In this binding mode CAS and PAS both were well occupied by the ligand and hence it is in agreement with the mixed mode inhibition pattern of $\mathbf{C 1 8}$ [47].

Saeed et al. [48] synthesized a series of coumarin linked thiourea derivatives and tested their potential inhibitory activity against $\mathrm{AChE}$ and BuChE. Among all the compounds synthesized specifically compounds 1-(2-Oxo-2H-chromene-3-carbonyl)-3-(3-chlorophenyl) thiourea (C20) and 1-(2-Oxo-2H-chromene-3-carbonyl)3-(2-methoxyphenyl)thiourea (C21) were found to be strongest inhibitors against $\mathrm{AChE}$ and $\mathrm{BuChE}$ with $\mathrm{IC}_{50}$ values of 0.04 and $0.06 \mu \mathrm{M}$, respectively (Fig. 28). Docking studies were performed to get the detail of the inhibitory behaviour and probable binding modes of all inhibitors against both cholinesterase using the standard drug donepezil with especial focus on the most active compounds.

Docking results concluded similar binding modes with different docking scores for mostly all of the compounds which were found to be well docked near the catalytic triad of AChE (Glu225, Ser226, His466) 

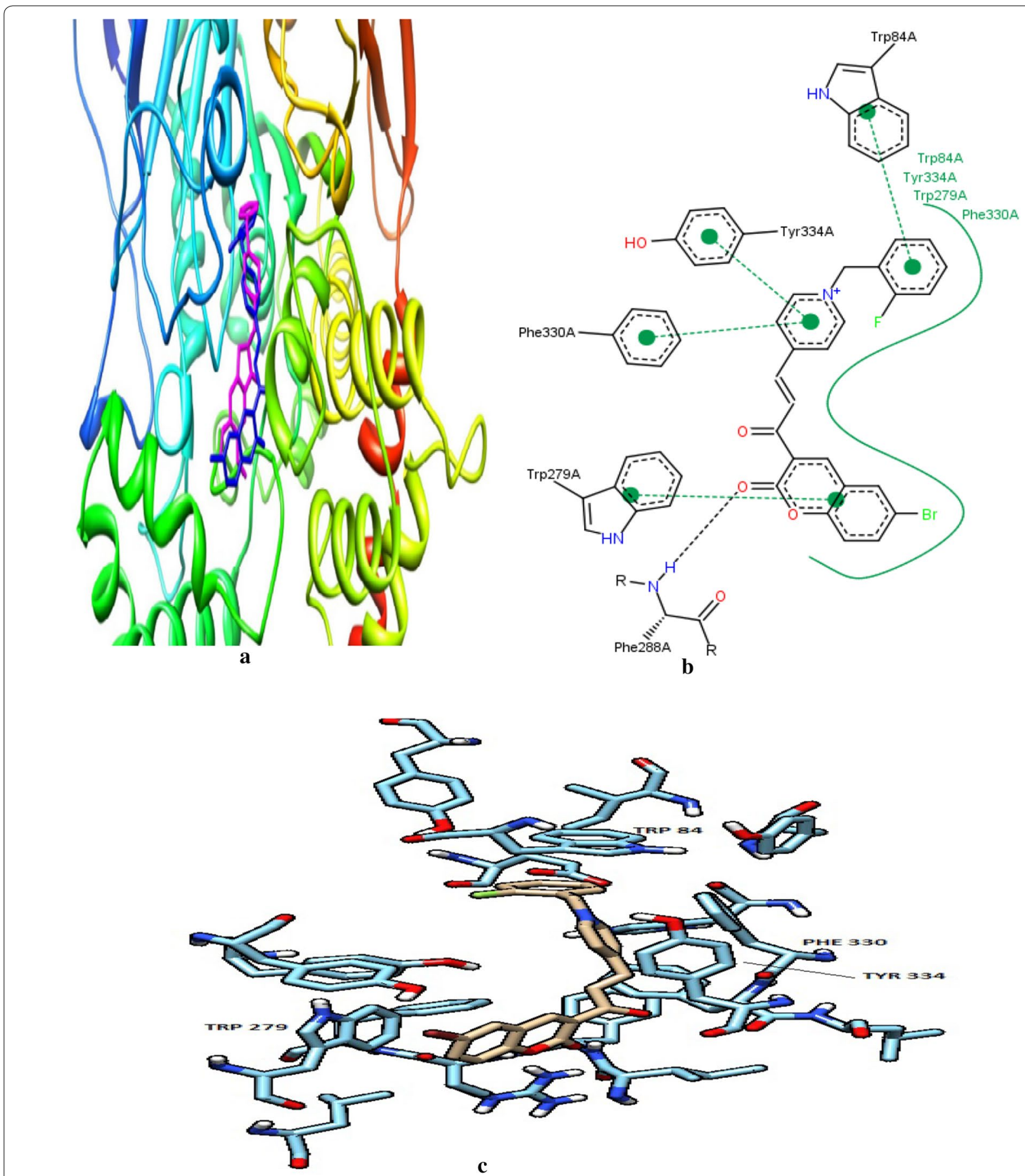

Fig. 22 a Superimposition of C15a (blue) and donepezil (magenta) in the gorge of TcAChE. $\mathbf{b} 2 \mathrm{D}$ representation of binding mode of C15a and amino acid residues in the gorge of TCAChE created by PoseView. $\mathbf{c}$ Docking of C15a in the active site of TcAChE [45]

forming hydrogen bond with Tyr146 via O-HAN interaction (Fig. 29a) but slightly different binding modes in the catalytic triad of BuChE (Glu224, Ser225, His 494) with no hydrogen bonding which might be due to their slightly different active site architecture (Fig. 29b) [48].
Shaik et al. [49] designed tricyclic coumarin derivatives bearing iminopyran ring connected to different amido moieties and talked in detail about their in vitro dual $\mathrm{AChE} / \mathrm{BuChE}$ inhibitory properties against galantamine, tacrine, donepezil and rivastigmine as positive controls 


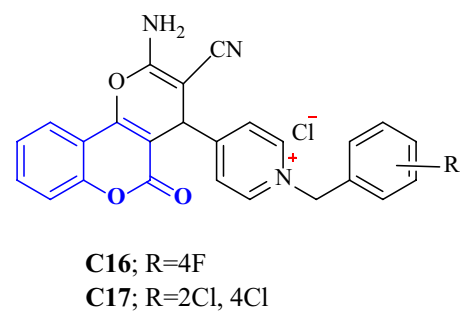

Fig. 23 Chemical structures for 5-oxo-4,5-dihydropyrano coumarin derivatives

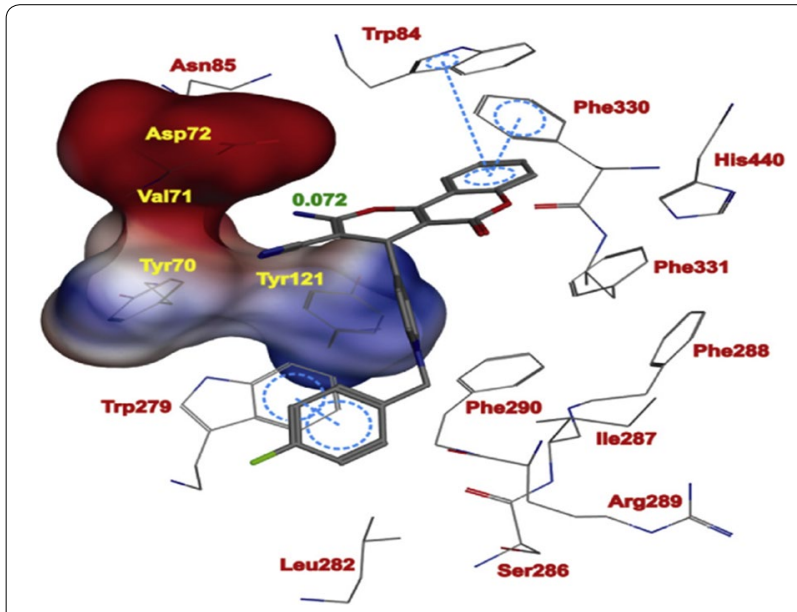

Fig. 24 A representative model for interaction of compound C16 and the AChE [46]

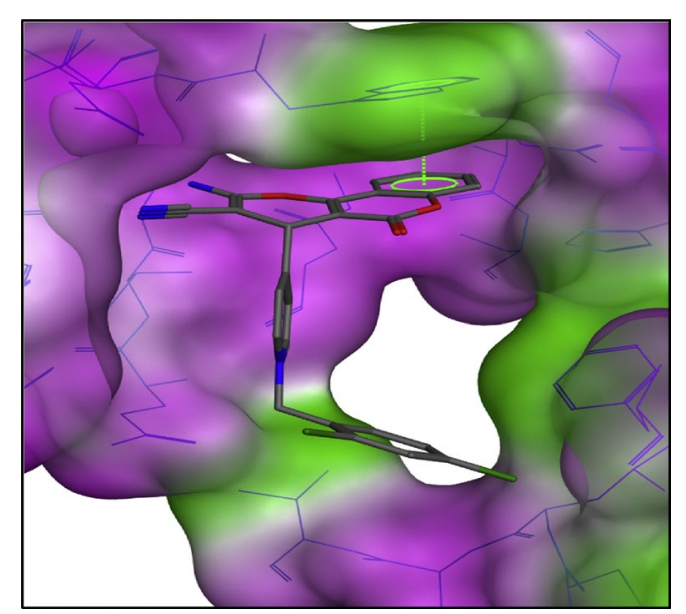

Fig. 25 The predicted binding mode of compound $\mathbf{C 1 7}$ in the active site of BuChE [46]

(Fig. 30). Compound C31 was specifically the most potent $\mathrm{AChE}$ inhibitor with $\mathrm{IC}_{50} 0.003 \mu \mathrm{M}$ and $\mathrm{C22}$ was the most potent inhibitor of BuChE with $\mathrm{IC}_{50} 11.32 \mu \mathrm{M}$.
Importantly, compounds C30, C31, C32, C33 and C34 were found to be overall stronger anti-AChE inhibitors than all the chosen reference compounds. Compounds C22, C23 and C24 were reported as dual inhibitors of both the enzymes. It was interesting to note the relation between the structure-activity relationships, and the effect of the substituents in phenyl ring of amide moiety in the enzyme inhibition. The cyclohexyl moiety (C25) on amido part was reported to increase the anti-AChE activity to about 35 -fold as compared to the cyclopropyl moiety (C23) on the amido part of the molecule. Elongation of the lipophilic side chain in compounds $\mathbf{C 2 6}$ $\left(\mathrm{IC}_{50}=1.4 \mu \mathrm{M}\right), \mathrm{C} 22\left(\mathrm{IC}_{50}=0.249 \mu \mathrm{M}\right)$ and C27 $\left(\mathrm{IC}_{50}\right.$ $0.022 \mu \mathrm{M})$ also increased the AChE inhibitory activity. The longer the aliphatic chain (C27) the stronger was its anti-AChE activity. On the other hand the BuChE inhibitory activity was limited to $N$-ethyl group from $N$-methyl group. By changing the aliphatic methyl group present at the benzylic junction (C28) with the aromatic phenyl ring (C29) at the same junction though increased the AChE activity but decreased the BuChE activity. The electron withdrawing substituent $(-\mathrm{Cl},-\mathrm{Br})$ present at othro or meta positions of the benzyl moiety made the compounds 110-700 fold more potent (C30, C31, C32, and C33) than compounds with unsubstituted benzyl moiety (C35). In addition to these compound C34 with para-(ter-butyl) benzyl moiety was also found to be 210 folds more potent than compound C35.

In order to resolve the factors affecting the AChE activity Schrödinger maestro software was used to investigate the possible binding modes of the top 5 anti-AChE compounds viz C30-C34 and therefore they were docked into the active site of the eelAChE. Compound C30, C32 and C34 were found to be similarly oriented into the active site of the enzymes whereas the binding modes of compounds C31 and C33 was found to be different from the binding modes of C30, C32 and C34. The orientation of compounds C30, C32 and C34 accommodated the ligand in the PAS in such a way, that the tricyclic coumarin ring was noted to be sandwiched between Trp286 and Tyr341 via $\pi-\pi$ stacking while the 2-bromo benzylamido moiety protruded to the opening of the PAS and was found bounded to Glu292 via $\mathrm{CH}-\pi$ interaction and a hydrogen bond interaction was achieved between the carbonyl moiety and the hydroxyl group of Phe295. In addition to these it was also noted importantly that compounds C30, C32 and C34 were extended outside the gorge of $\mathrm{AChE}$, supporting the feature, for the AChE-induced $A \beta$ aggregation inhibition. The orientation pose of ligands C31 and C33 were reversed to that of C30, C32 and C34. These ligands was found to be nicely accommodated in the gorge of AChE active site, in such a manner that the benzylamido moiety was noted to 


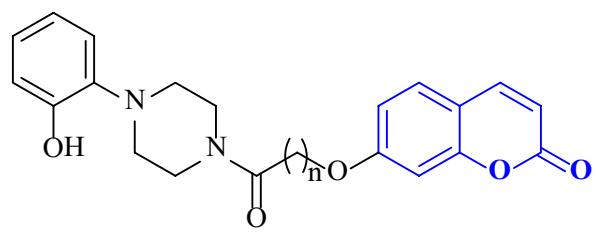

C18

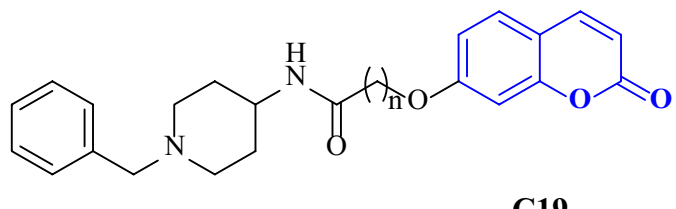

C19

Fig. 26 Benzylpiperidinylamino derivative $\mathbf{C 1 8}$ and (2-fluorophenyl)piperazine derivative C19

lean towards CAS. A T-shape edge-to-face $\pi-\pi$ stacking interaction of, 3-halo phenyl ring of benzylamido moiety against Phe338 was observed specifically which helped in hydrogen bond interaction with the $\mathrm{OH}$ of Tyr124 inside the mid gorge. In this binding mode CAS and PAS both were well occupied by the ligand and hence it is in agreement with the mixed mode inhibition pattern of $\mathbf{C} 31$ but however on the other hand compound C33 disclosed only one hydrogen bond interaction between imino $\mathrm{NH}$ and Phe 295 (Fig. 31).

The AChE is composed of aromatic amino acid residues like Tyr72, Tyr124, Trp286, Phe295, Phe297 and Tyr337 and BuChE is composed of aliphatic amino acid residues like Asn68, Gln119, Ala277, Leu286, Val288, and Ala328. This is the cause factor behind the poor ability of BuChE to form $\pi-\pi$ stacking interactions as compared to AChE. Docking studies of the most active anti-BuChE compound (C22) revealed that it can effectively fit into the gorge of BuChE active site which makes it a more potent $\mathrm{BuChE}$ inhibitor than other member of the series. Its favourable orientation within the gorge accounts for higher affinity towards BuChE and effective $\pi-\pi$ stacking interaction between Phe357 and tricyclic coumarin moiety. In addition, two hydrogen bonds were reported with the amino acids of the catalytic triad. One between the oxygen of coumarin and $\mathrm{OH}$ of Ser226 and the other between the carbonyl of coumarin and $\mathrm{NH}$ of His446. A $\mathrm{CH}-\pi$ interaction between Trp110 and $\mathrm{N}$-ethyl group of the amide part was also reported. Moreover hydrophobic interactions with Trp110, Leu313, Leu314, Val316, Ala356, Ile426, Trp458, Met465, and Tyr468 aliphatic residues were also reported (Fig. 32) [49].

Alipour et al. [50] synthesized 3-coumaranone and coumarin derivatives encompassing the phenacyl pyridinium moiety as dual inhibitors of acetyl and butyrylcholinesterase. The obtained docking results suggested that all the synthesized compounds were dual binding inhibitors of $\mathrm{AChE}$ in the micromolar range with slightly different interactions with the receptor. Interestingly it was reported that 3-coumaranone derivatives were more good AChE inhibitors than the coumarin derivatives (Fig. 33).
Taking into account the results obtained from the coumarin derivatives, it was revealed that coumarin analogues were protruded towards the CS and the longer molecules were reported to stretch out of the gorge and found interacting with Trp84 at the proximity of the CS via $\pi-\pi$ interaction (Fig. 34).

In terms of structure activity relationship the order of activity was immensely affected by the electron withdrawing nature and lipophilicity of the substituents present on the penacyl pendant which followed the activity order as: $4-\mathrm{F} \approx 4-\mathrm{H}>4-\mathrm{CH} 3>4-\mathrm{OCH} 3>4-\mathrm{Br}$ (C36, C37, C38, C39, C40). The orientation of the phenacyl pendant is directed towards the hydrophilic pocket comprised of Gly441, Glu199, His440, and Tyr130. Therefore the substituents with strong lipophilic nature at para position of phenacyl ring (4-Br) are imperfectly accommodated within the lipophilic pocket, making the analogue a slow AChE inhibitor (C40) (Fig. 35). Electron withdrawing substituents which were low in lipophilic character (4-F) were found to be well tolerated at para position of phenacyl ring as reported for compound C36. On the other hand, substituents with good electron donating nature $\left(4-\mathrm{CH}_{3}, 4-\mathrm{OCH}_{3}\right)$ at para position were reported to diminish AChE activity by weakening the $\pi-\pi$ interaction with Trp84 (C38, C39). Moreover, coumarin analogues were found to be slow butyrylcholinesterase inhibitor as compared to the inhibition of AChE except for the derivative $\mathbf{C 4 0}$ with methyl substituent at para position displaying the $\mathrm{IC}_{50}$ value of $8 \mu \mathrm{M}$ [50].

Catto et al. [51] reported a series of coumarin alkylamines (C41a-C41s; Entry 1-19), matching the structural determinants to one of the most commonly used standard marketing anti-AChE drug donepezil, as potent and dual binding site inhibitors for acetylcholinesterase (Figs. 36, 37, 38 and 39). Among the synthesized series, the 6,7-dimethoxycoumarin analogues which were composed of protonatable benzylamino group and linked via suitable linker to position-3 were found to be the most active AChE inhibitor as well as high selective over $\mathrm{BuChE}$. It's worth to mention here that the extent of inhibition was influenced by the length and the shape of the spacer and by the presence of methoxy substituents on the coumarin nucleus. The most active compound $\mathbf{C 4 1 m}$ 


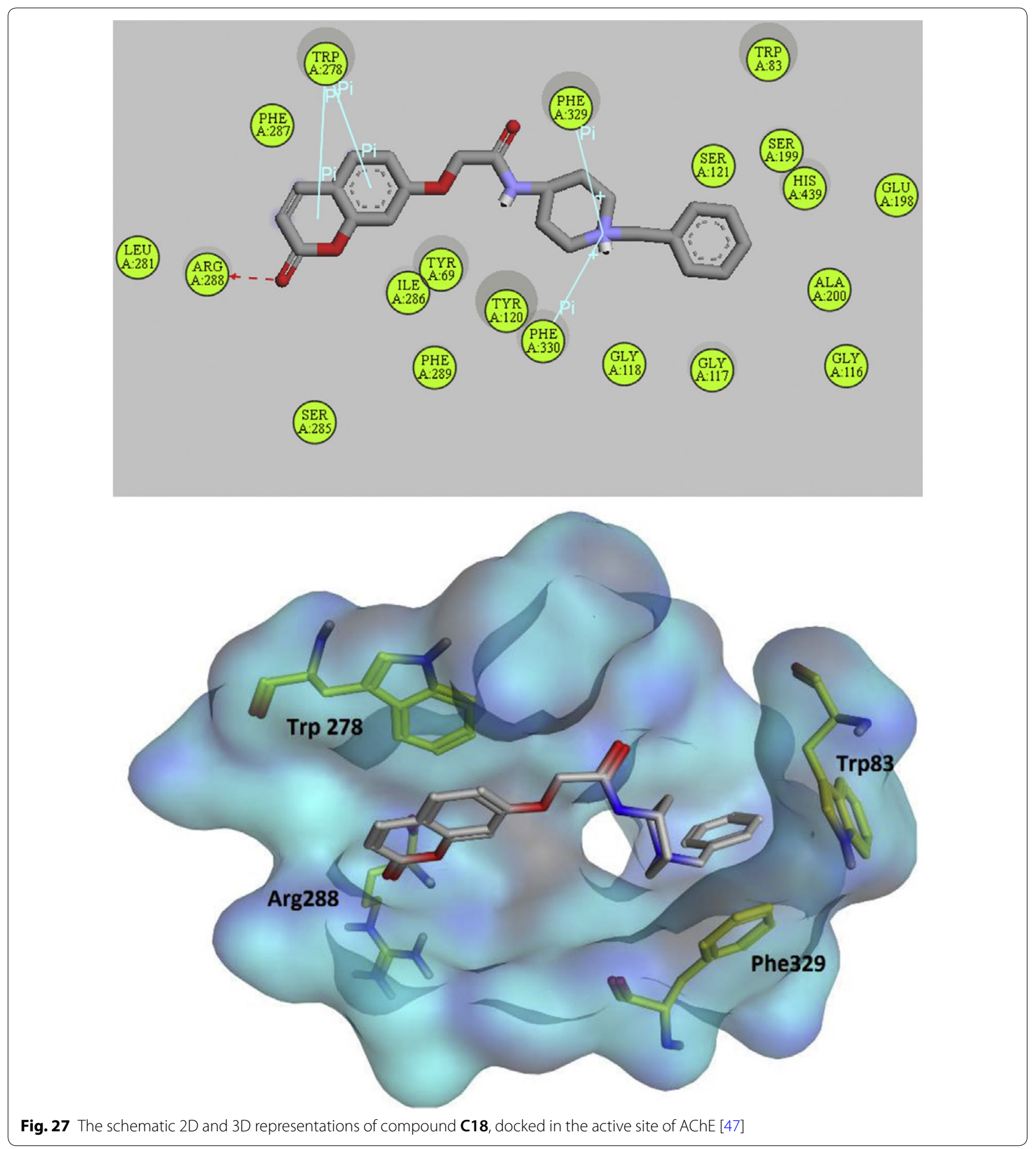

(entry 13) with $\mathrm{IC}_{50}$ value of $7.6 \mathrm{nM}$ was found to be the dual inhibitor revealing binding at the CS and PAS of AChE enzyme.

In order to get the insight behaviour of the coumarin analogues anti-ChE activities were performed on $\mathrm{AChE}$ collected from bovine erythrocytes and BuChE collected from equine serum by Ellman's methodology. Results suggested that all the compounds except aminoethers C41n, C41o and C41p (entry 14, 15 and 16) were selective $\mathrm{AChE}$ inhibitor as compared to $\mathrm{BuChE}$ with $\mathrm{IC}_{50}$ values in the range of $66 \mu \mathrm{M}$ for compound C41j (entry 10) to $7.6 \mathrm{nM}$ for the cis-3-amino-cyclohexanecarboxylic 
<smiles>O=C(NC(=S)Nc1cccc(Cl)c1)c1cc2ccccc2oc1=O</smiles>

$\mathrm{C20}$<smiles>COc1ccccc1NC(=S)NC(=O)c1cc2ccccc2oc1=O</smiles>

C21
Fig. 28 Chemical structure for thiourea coumarin derivatives C20 and $\mathbf{C 2 1}$

acid derivative $(\mathbf{C 4 1 m})$ whose $\mathrm{IC}_{50}$ value was the highest and very close to the reference donepezil, with $\mathrm{IC}_{50}$ 4.2 nM. Additionally compounds C41a-C41c (entry 1-3) also showed an increase in potency with the elongation of their polymethylene linker. Compound C41a $(9.0 \mu \mathrm{M})$ was the less active compound from the amide series and the $\mathrm{IC}_{50}$ was noted to decrease on moving to the least compound C41b (86 nM) whilst compound C41c was the most active compound (21 nM). Compound C411 (entry 12), the amide analogue of compound C41b, was reported with the four-fold drop of inhibitory activity due to the presence of inverted amide function. On the other hand compound C41i (entry 9) with short methylene linker and aminoether compound C41n (entry 14) with longer triethylene linker was reported to exhibit low inhibition. The absence of two-methoxy group in positions 6 and 7 of the coumarin ring might the cause of this decrease AChE affinity and a total loss of AChE/BChE selectivity for compound C41n. The closely related compound $\mathbf{C 4 1 n}\left(\mathrm{IC}_{50}=4.5 \mu \mathrm{M}\right.$, entry 14) and C41o $\left(\mathrm{IC}_{50}=4.5 \mu \mathrm{M}\right.$, entry 15$)$ were found to be equally potent anti-AChE while the monomethoxy substituted analogues C41p $\left(\mathrm{IC}_{50}=7.4 \mu \mathrm{M}\right.$, entry 16) and C41q $\left(\mathrm{IC}_{50}=12 \mu \mathrm{M}\right.$, entry 17$)$ were noted to be weaker but displaying moderate selectivity over BuChE. Moreover 6,7-dimethoxy analogue C41r $\left(\mathrm{IC}_{50}=1.5 \mu \mathrm{M}\right.$, entry 18) expressed low inhibitory property but good BuChE selectivity. It was noted that the elongation of spacer with a methylene group, from derivative C41r to derivative C41s $\left(\mathrm{IC}_{50}=21 \mathrm{nM}\right.$, entry 19$)$ increased its potency as well as increased its $\mathrm{BuChE} / \mathrm{AChE}$ selectivity ratio by 186. The inhibition mechanism of the enzyme AChE by the most active compound $\mathbf{C 4 1 m}$ was also interpreted by the kinetic studies and the results are displayed via the Lineweaver-Burk plot (Fig. 40). The inhibition constant $\mathrm{Ki}$, was equal to $8.6 \pm 1.5 \mathrm{nM}$. The plot displayed reversible and mixed type inhibition model, which was in accordance with the dual binding site model of interaction with the enzyme AChE [51].

Researcher Leonardo Pisani from the above mentioned research group of Catto et al. also designed and synthesized another large series of coumarin derivatives. Pisani et al. linked the coumarin ring to 3-hydroxy- $N, N$-dimethylanilino or 3-hydroxy- $N, N, N$-trialkylbenzaminium moieties via a suitable spacer and subjected the derivatives for their further evaluation as acetylcholinesterase (AChE) and butyrylcholinesterase (BuChE) inhibitors (Fig. 41).

Among all the derivatives the one with two methoxy groups at position 6 and 7 of the coumarin ring viz 3-\{4-[3-(Dimethylamino)-5-hydroxyphenoxy] butoxy\}6,7-dimethoxy-2H-chromen-2-one (C43), was dramatically found to be the most potent anti-AChE displaying $\mathrm{IC}_{50} 0.236 \mathrm{nM}$ even higher than the chosen reference donepezil. Compound $\mathbf{C 4 3}$ and donepezil both were reported to exhibit higher $\mathrm{IC}_{50}$ values towards human $\mathrm{AChE}\left(\mathrm{IC}_{50}=39.7 \mathrm{nM}\right)$ as compared to bovine $\mathrm{AChE}$ $\left(\mathrm{IC}_{50}=26.0 \mathrm{nM}\right)$. Even so compound $\mathrm{C43}$ was an

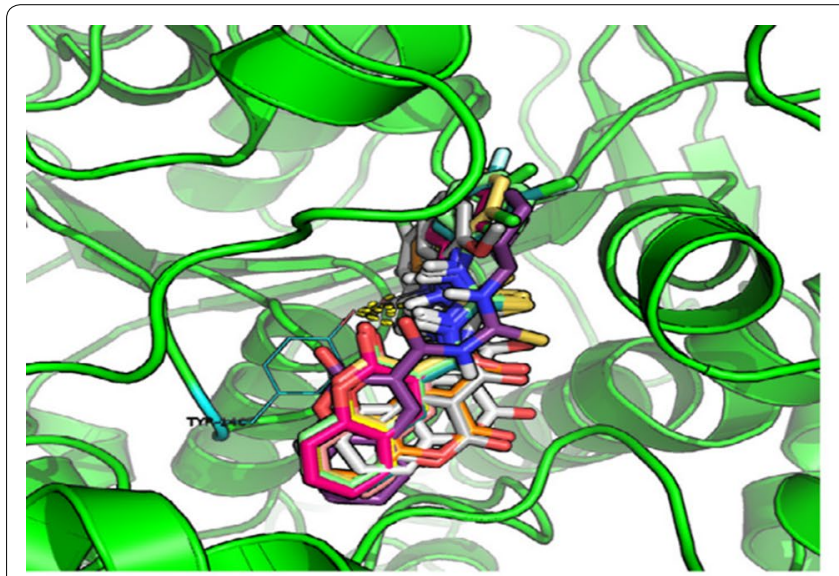

a

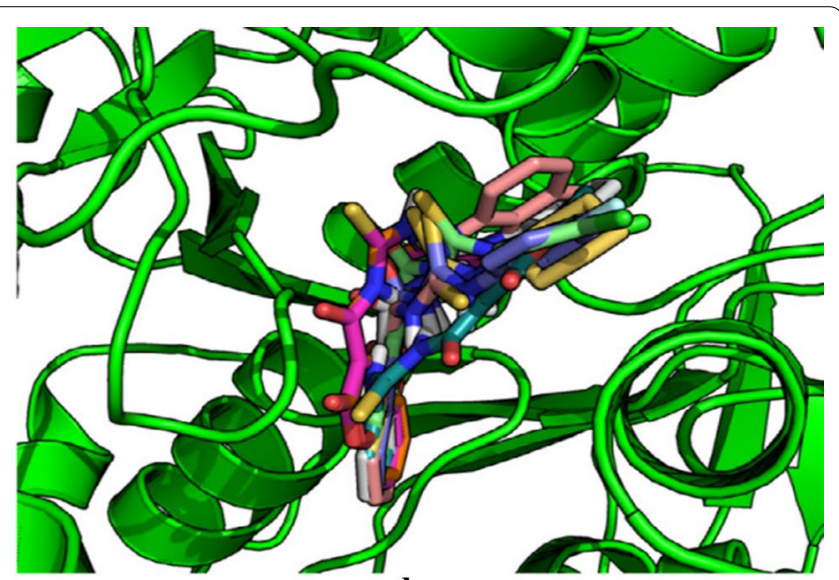

b

Fig. 29 a, b Binding models of the synthesized compounds in AChE and BuChE enzymes active sites. Yellow dashed lines in (a) showing hydrogen bonding between Tyr146 and one of thioureas hydrogen atom from different compounds [48] 
<smiles>[R]N([R])C(=O)c1cc2c(ccc3c(C)cc(=O)oc32)oc1=N</smiles>

C22; $\mathrm{R}_{1} \mathrm{R}_{2} \mathrm{~N}=\mathrm{NHCH}_{2} \mathrm{CH}_{3}$

C23; $\mathrm{R}_{1} \mathrm{R}_{2} \mathrm{~N}=-\mathrm{NH} \longrightarrow$

C24;<smiles>[R12]N=[W]</smiles>

C25; $\mathrm{R}_{1} \mathrm{R}_{2} \mathrm{~N}=-\mathrm{NH} \longrightarrow$

C26; $\mathrm{R}_{1} \mathrm{R}_{2} \mathrm{~N}=\mathrm{NHCH}_{3}$

C27; $\mathrm{R}_{1} \mathrm{R}_{2} \mathrm{~N}=\mathrm{NH}\left(\mathrm{CH}_{2}\right)_{11} \mathrm{CH}_{3}$

$\mathrm{C28} ; \mathrm{R}_{1} \mathrm{R}_{2} \mathrm{~N}=-$
C30;<smiles>[R12]N=[W]</smiles><smiles>[R16]N=[W]</smiles><smiles>[R16]N=[W]</smiles>

C33; $\mathrm{R}_{1} \mathrm{R}_{2} \mathrm{~N}=\mathrm{HN}^{\mathrm{Cl}}$<smiles></smiles><smiles>[R16]C(=NCc1ccccc1)NC</smiles>

Fig. 30 Chemical structures of tricyclic coumarin derivatives C22-C35

excellent $\mathrm{AChE}$ inhibitor, unexpectedly its affinity profile towards $\mathrm{BuChE}$ was quite poor $\left(\mathrm{IC}_{50}=71,000 \mathrm{nM}\right)$. Same ways the activities of all the derivatives was checked and the $\mathrm{BuChE}$ and $\mathrm{AChE}$ inhibition data suggested that these compounds could be regarded as selective, sub-micromolar AChE inhibitors rather than potential BuChE inhibitors. Therefore, the surprisingly high AChE binding affinity observed in moving from inhibitor C42, (3-\{4-[3-(Dimethylamino)-5-hydroxyphenoxy] butoxy\}-2H-chromen-2-one) to $\mathbf{C 4 3}\left(\mathrm{IC}_{50}=143 \mathrm{nM}\right)$, 

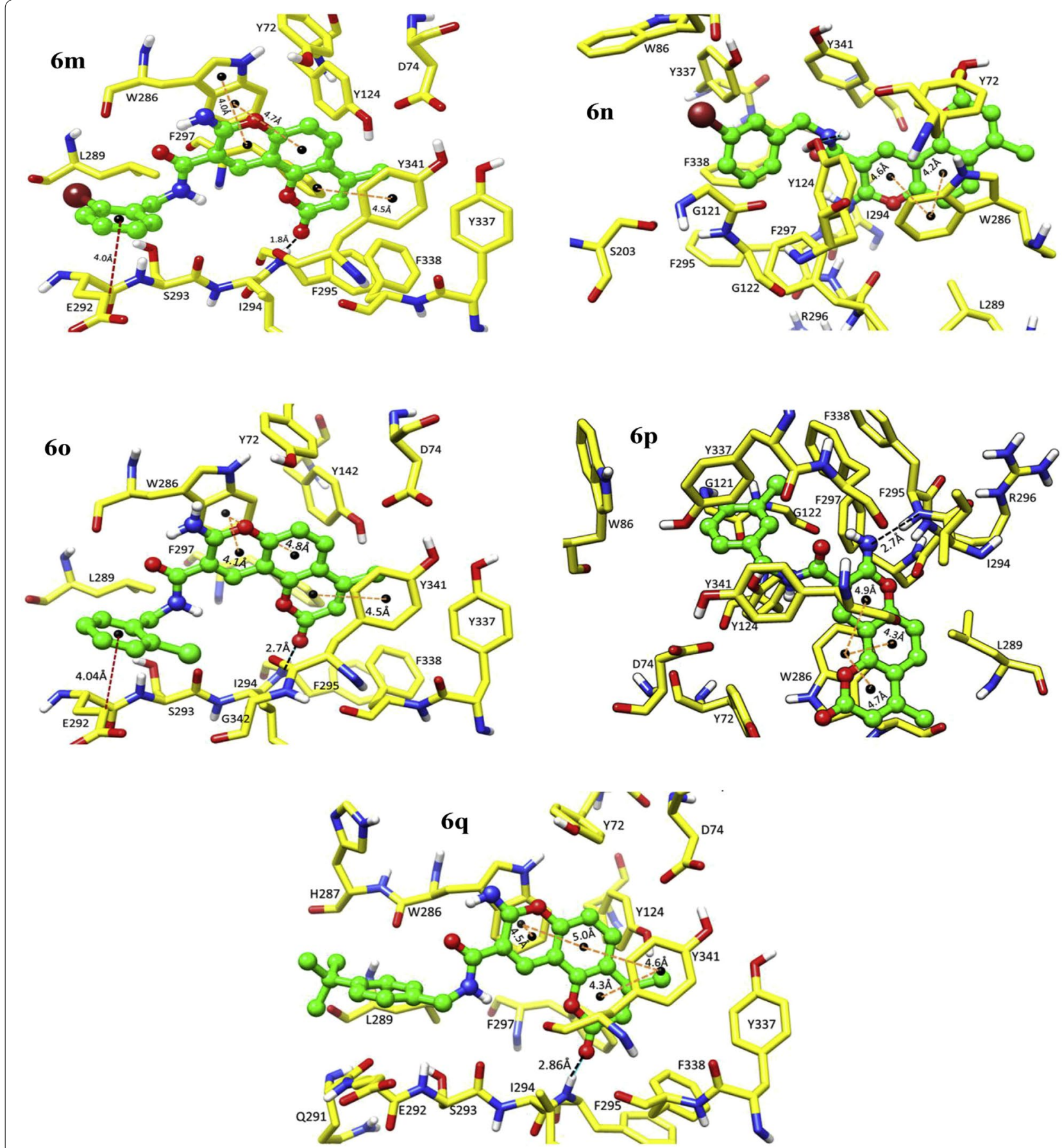

Fig. 31 Docking pose of compounds C30-C34 at the binding site of AChE [49]

which differs only in the 6,7-dimethoxy substitution at the coumarin ring was tried to investigated by molecular docking (Fig. 42). But unfortunately docking results were incapable to interpret this unexpected rise in $\mathrm{AChE}$ binding affinity for inhibitor C43. Hence, the MD study was performed.
The top-scored docking poses of these two reference compounds were took into account for the MD studies to determine the variation in structure in the AChE complexes over time. Taking about the inhibitor C43 first, an overall sandwich-like orientation was maintained throughout the MD procedure due 


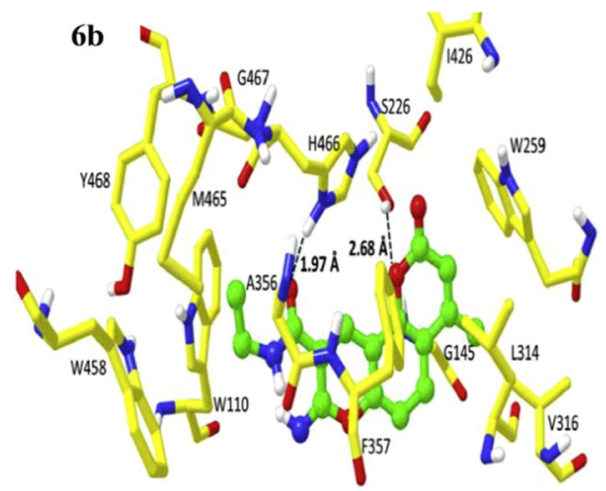

Fig. 32 Docking pose of compounds C22 at the binding site of BuChE [49]

to stabilizing $\pi-\pi$ stacking interaction which was established because of the two methoxy substituents which stabilized the coumarin moiety into the PAS at the place whose large area was exposed to the solvent. These two methoxy groups which were facing the solvent, enabled inhibitor C42, to sandwiched its coumarin moiety into the open slot between indolic and phenolic rings of Trp286 and Tyr341, respectively (Fig. 43). In the primary binding site of the enzyme $\mathrm{AChE}$, the inhibitor $\mathbf{C 4 3}$ was reported to orthogonally orient its 3-hydroxy- $N, N$-dimethylanilino fragment to Trp86 and thus forming network of hydrogen bonds with the two solvent water molecules through their $N, N$-dimethylamino and phenolic group. On the other hand, inhibitor $\mathbf{C 4 2}$ was reported to be smarter to dive deeper in the gorge of AChE in order to diminish the exposure to the solvent. However for $\mathbf{C 4 2}$ the sandwich interaction with Trp286 and Tyr341 was not reported because the coumarin ring was found to form a T-shaped orthogonal $\pi-\pi$ interaction with $\operatorname{Trp} 286$ or, a parallel - $\pi$ interaction with Tyr341 [52].

To interpret the pronounced molecular enzymatic selectivity a comparison between AChE and BuChE topscored solutions of inhibitor $\mathbf{C 4 3}$ was done. The results revealed that, 3-hydroxy- $N, N$-dimethylanilino moiety was orthogonally oriented to Trp82, and its phenolic and ethereal oxygen atoms were reported to interact with Glu197 and Ser198 (BuChE numbering) of the catalytic triad by hydrogen bonds. Moreover, due to absebnce of PAS in BuChE possibility of $\pi-\pi$ interaction is not present as for AChE. Hence, the AChE/BuChE selectivity could be expressed via different molecular binding conformations observed and it is supported by the different energy scores (i.e. $57.27 \mathrm{Vs} 50.84 \mathrm{~kJ} / \mathrm{mol}$ ).

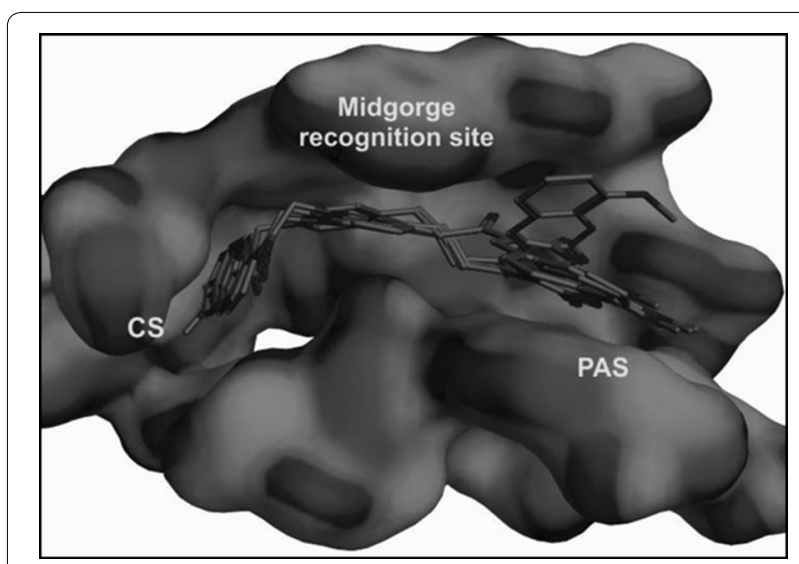

Fig. 34 Superimpositions of the best-docked poses of coumarin analogues in the AChE binding site [50]
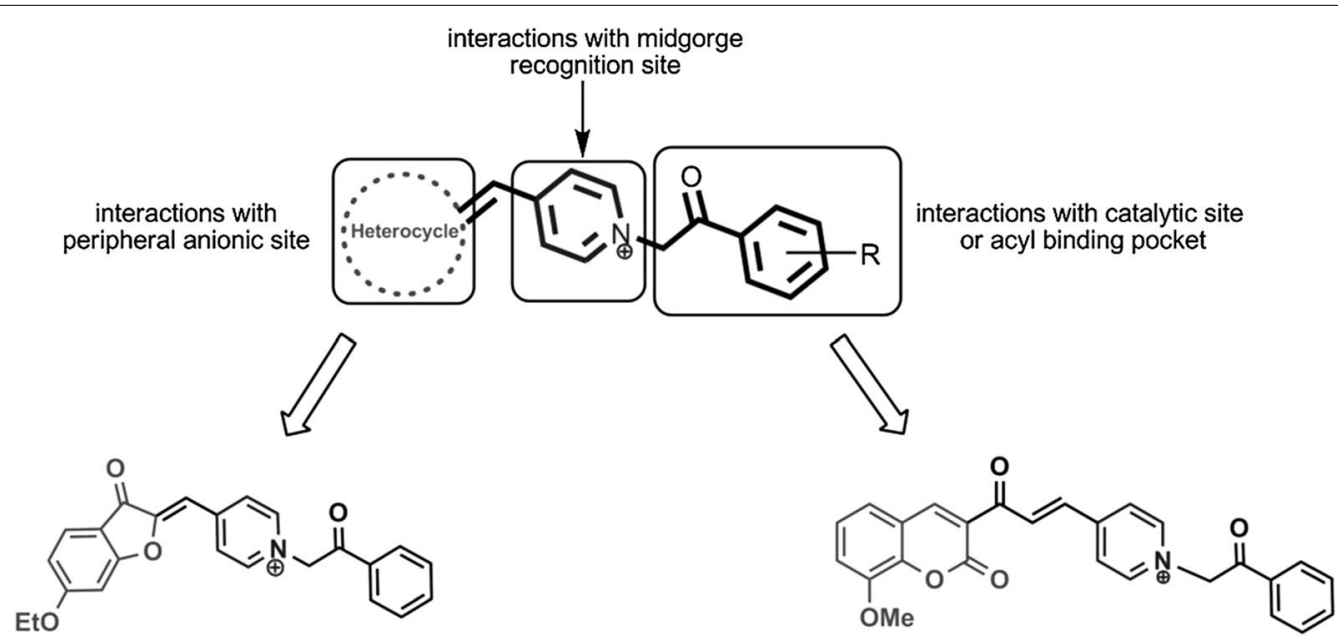

Fig. 33 Designed structures as potential inhibitors of AChE and BuChE [50] 


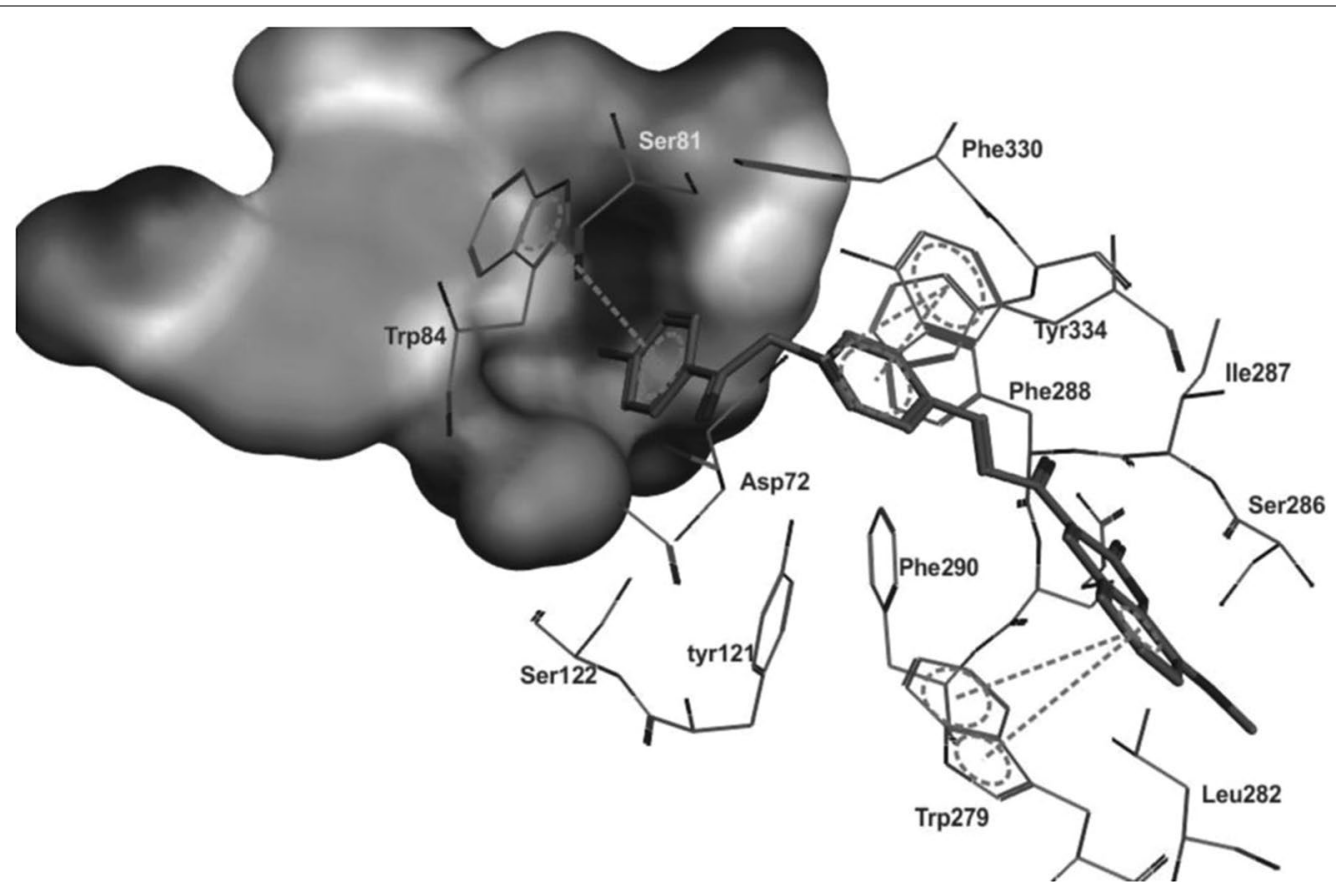

Fig. 35 Interaction of compound $\mathbf{C 4 0}$ docked into the binding site of AChE. The hydrophobic bromo substituent of $\mathbf{C 4 0}$ is oriented toward a hydrophilic pocket of active site [50]<smiles>COc1cc2c(cc1OC)C(=O)C(CC1CCN(CC3CCCCC3)CC1)C2</smiles>

$\mathrm{IC}_{50}$ AChE: $100-200 \mathrm{nM}$ $\mathrm{IC}_{50}$ BuChE: $20-40 \mathrm{nM}$<smiles>CCOc1ccc2c3c1O[C@@H]1C[C@@H](O)C=C[C@]31CCN(C)C2</smiles>

Galantamine<smiles>Nc1c2c(nc3ccccc13)CCCC2</smiles>

Tacrine

$\mathrm{IC}_{50}$ AChE: $0.7-1.5 \mu \mathrm{M}$

$\mathrm{IC}_{50}$ BuChE: $0.3-1.0 \mu \mathrm{M}$

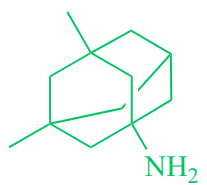

Memantine (NMDA antagonist)<smiles>C[C@H](c1cccc(OC(=O)N(C)C)c1)N(C)C</smiles>

Rivastigmine

$\mathrm{IC}_{50}$ AChE: 0.3-0.7 $\mu \mathrm{M}$

$\mathrm{IC}_{50}$ BuChE: $7-20 \mu \mathrm{M}$

IC $_{50}$ AChE: $2-10 \mathrm{nM}$

$\mathrm{IC}_{50}$ BuChE: 2-10 nM

Fig. 36 Marketed anti-AD drugs. For cholinergic drugs, inhibitory activity ranges on AChE and BuChE are reported

Ghanei-Nasab et al. [53] synthesized a series (C44a-C44o) of $N$-(2-(1H-indol-3-yl)ethyl)-2-oxo$2 \mathrm{H}$-chromene-3-carboxamides bearing tryptamine moiety and tested them against AChE and BuChE. The
SAR study revealed few facts about the presence of halogen atoms. One or two chlorine atoms on the benzyl moiety tend to decrease the anti-AChE property of the compounds whereas the flouro atom at ortho or meta 


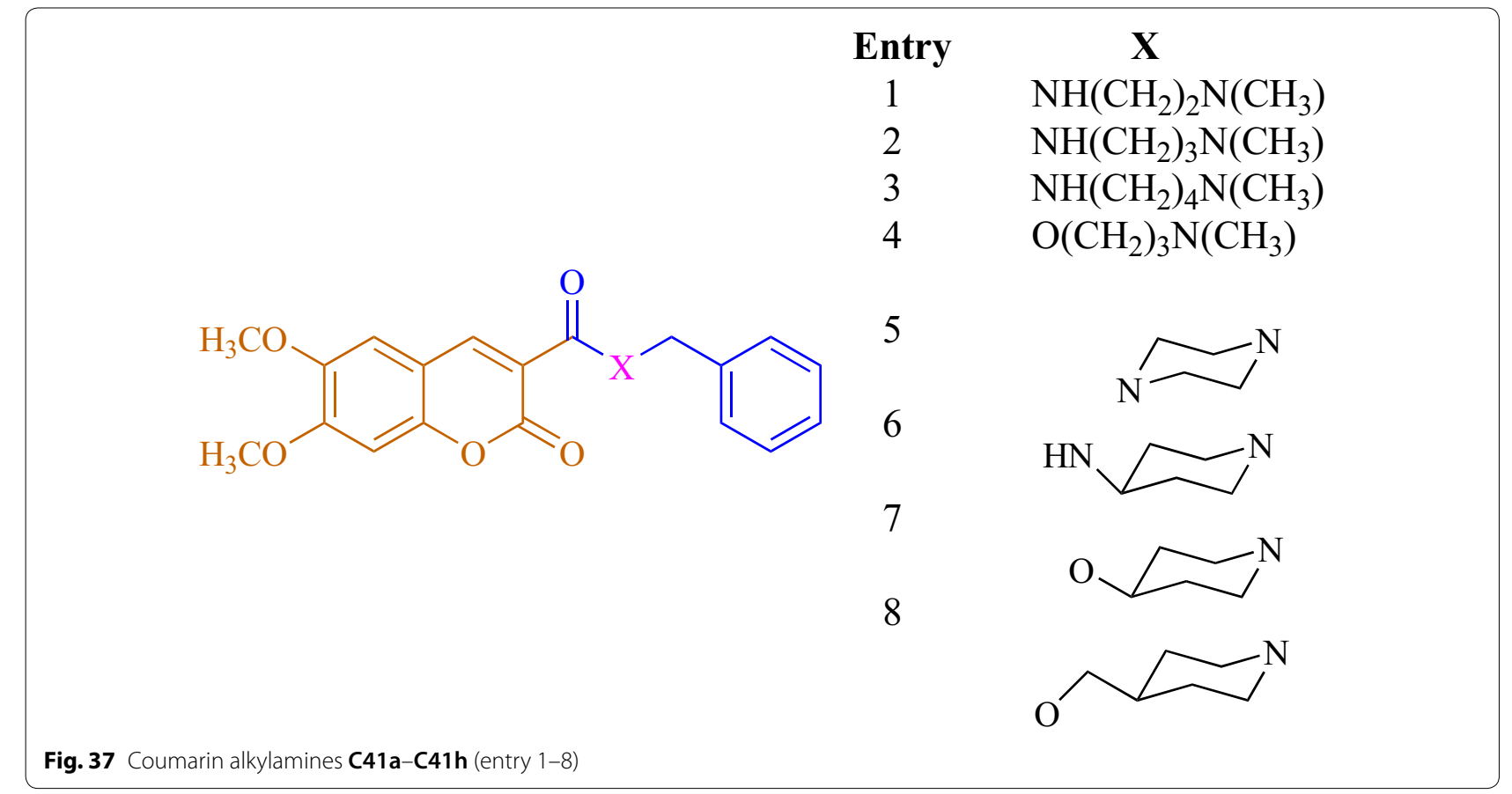

Fig. 38 Coumarin alkylamines C41i-C41I (entry 9-12)

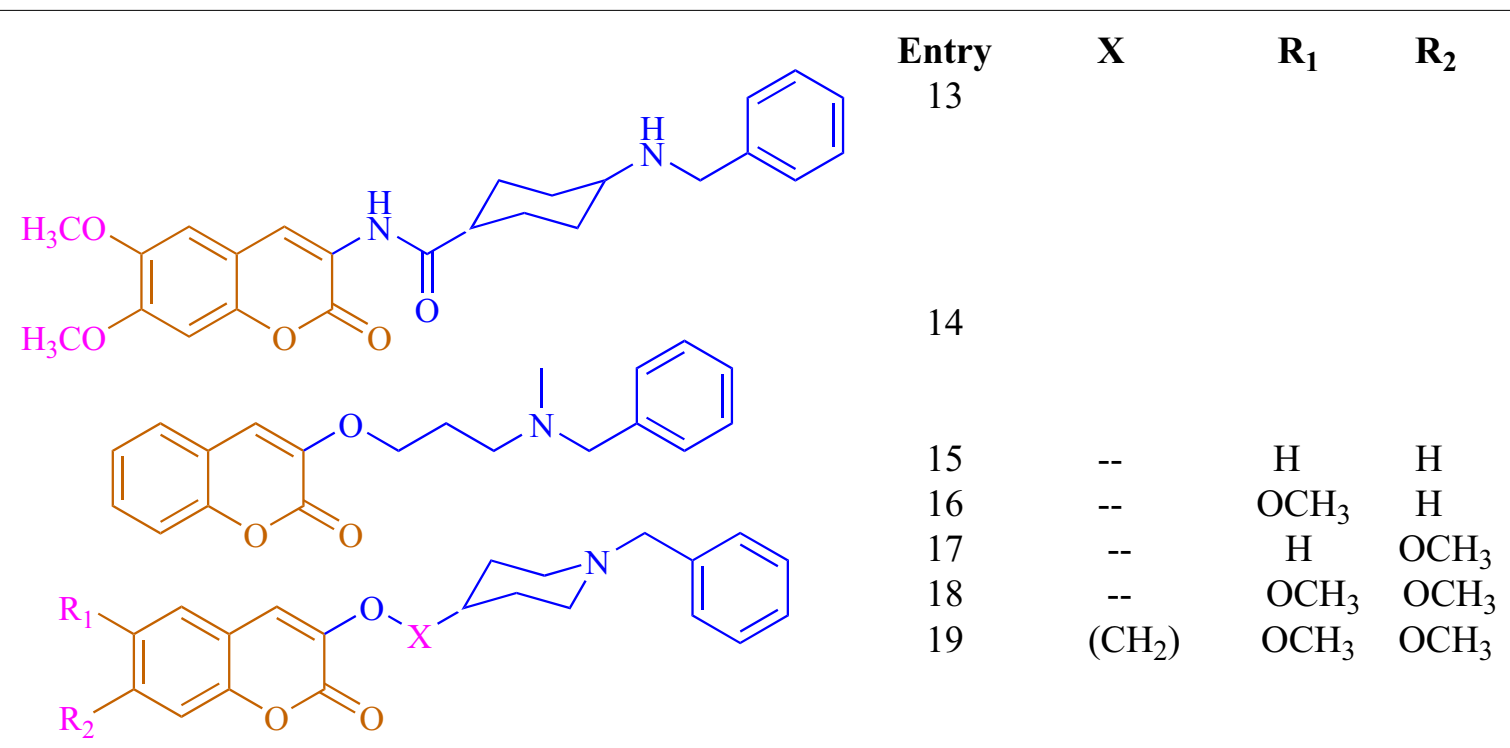

Fig. 39 Coumarin alkylamines C41 m-C41s (entry 13-19) 


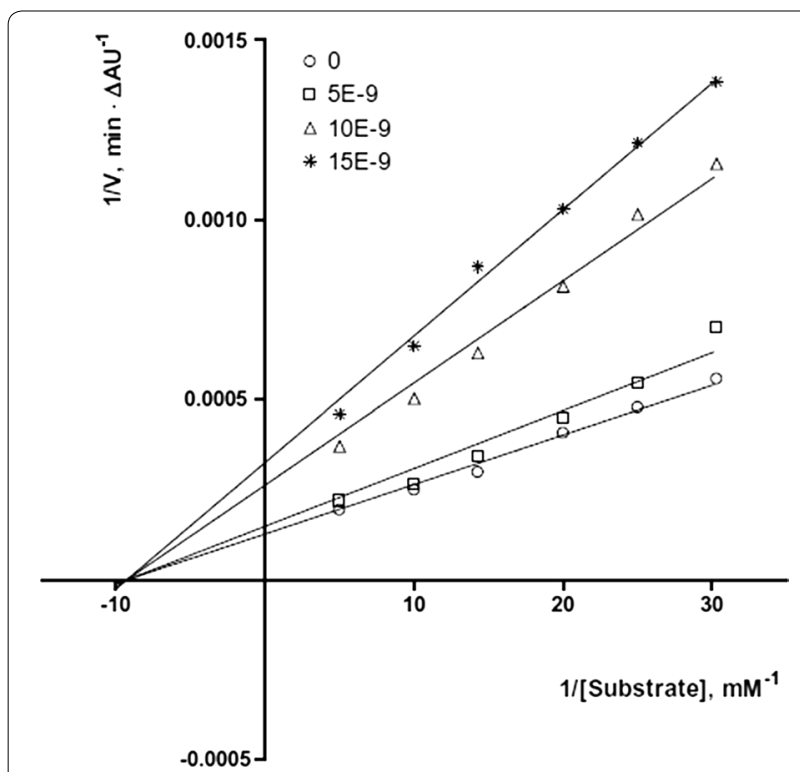

Fig. 40 Lineweaver-Burk plot of inhibition kinetics of $\mathbf{C 4 1}$ (entry 13): reciprocals of enzyme activity (bovine $\mathrm{AChE}$ ) vs reciprocals of substrate (S-acetylthiocholine) concentration in the presence of different concentrations (0-15 nM) of inhibitor C41 m (entry 13) [51]

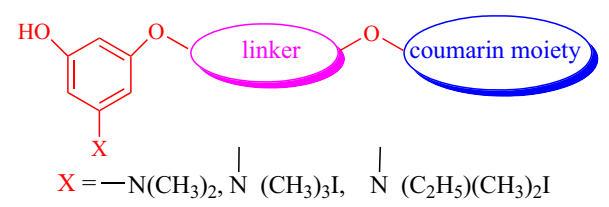

Fig. 41 General structure of the synthesized coumarin derivatives

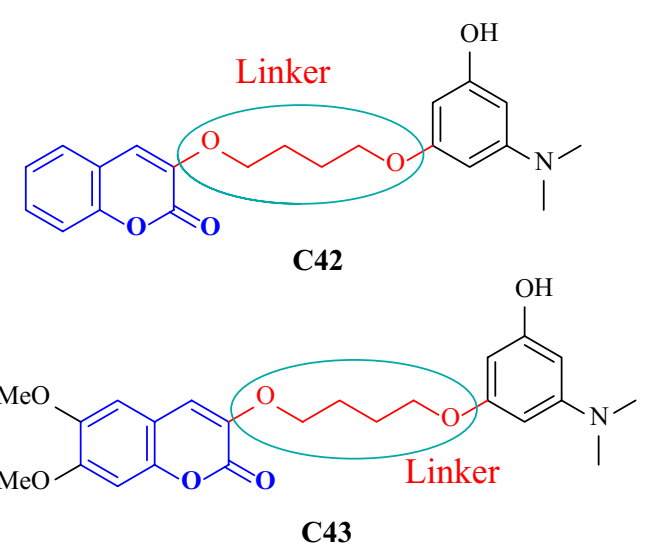

Fig. 42 Molecular structures of dimethylamino coumarin derivatives C42 and C43

position on the benzyl moiety was acting moderately and similarly (compounds C44m, C44n), but it was found to show improved activity against AChE when at para position (C44o). The most active compound C44o with flouro at para position of benzyl moiety was 15 -fold more stronger $\left(\mathrm{IC}_{50}=0.016 \mu \mathrm{M}\right)$ than $\mathbf{C 4 4 m}$ and $\mathbf{C 4 4 n}$ and 9-times superior to its benzyl analogue $\mathbf{C 4 4 h}$. The activity results for BuChE revealed that these compounds were mild or not active and the inhibitory activity of all compounds against $\mathrm{AChE}$ was higher over BuChE. The most potent $O$-benzyl derivative, $\mathbf{C 4 4 h}$ was found display $\mathrm{IC}_{50}$ value of $16.2 \mu \mathrm{M}$. It was 3 -fold more potent than analogue C44a (Fig. 44).

In order to get the insight of the binding modes the most active derivative C44o was laid across the active site and interestingly occupied PAS and CAS, both (Fig. 45). Its 4-fluorobenzyl moiety was found to be oriented towards the bottom of the active site and formed a faceto-face $\pi-\pi$ stacking with $\operatorname{Trp} 84$. $\pi-\pi$ stacking between coumarin ring and Tyr334 was also observed and indole ring was also found to interact with Trp279 of the PAS via $\pi-\pi$ interaction [53].

Hamulakova et al. [54] designed and synthesized acridine-coumarin hybrids and check their in vitro

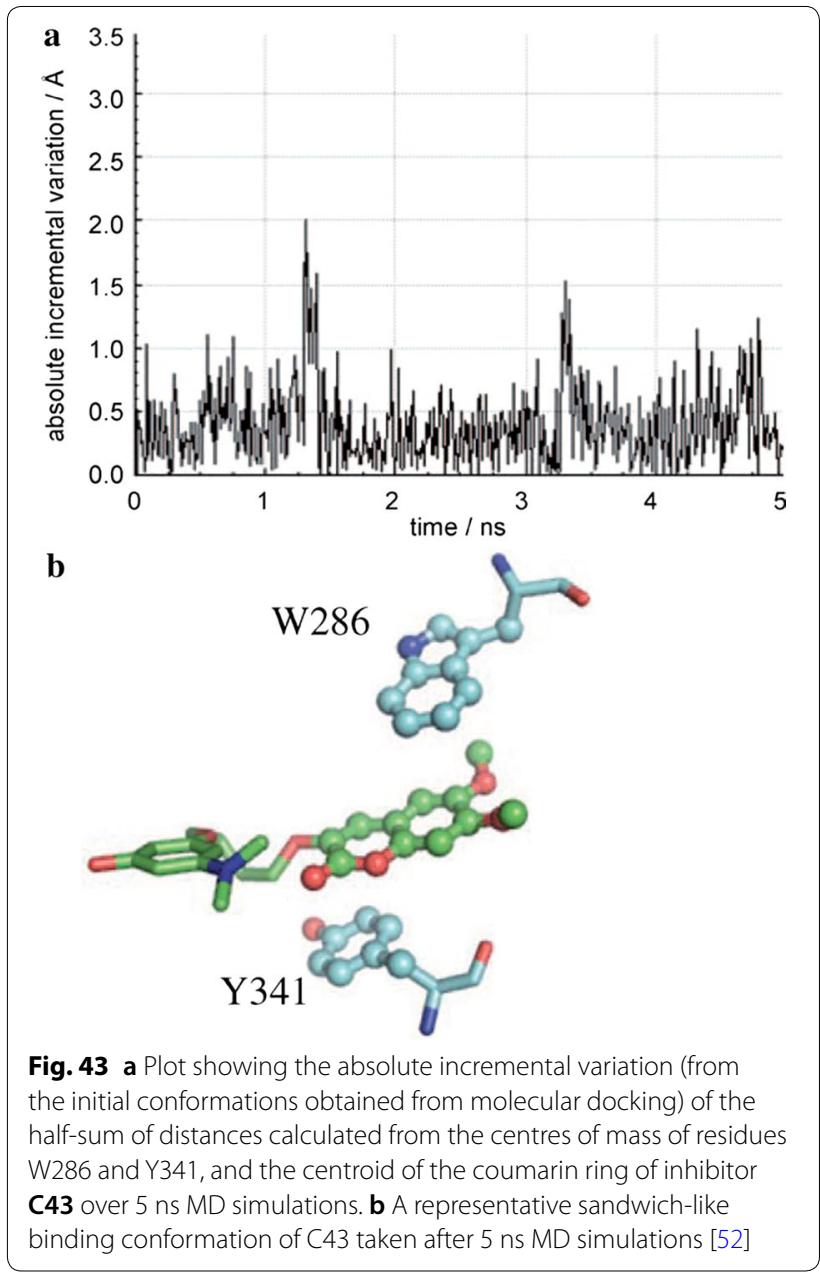




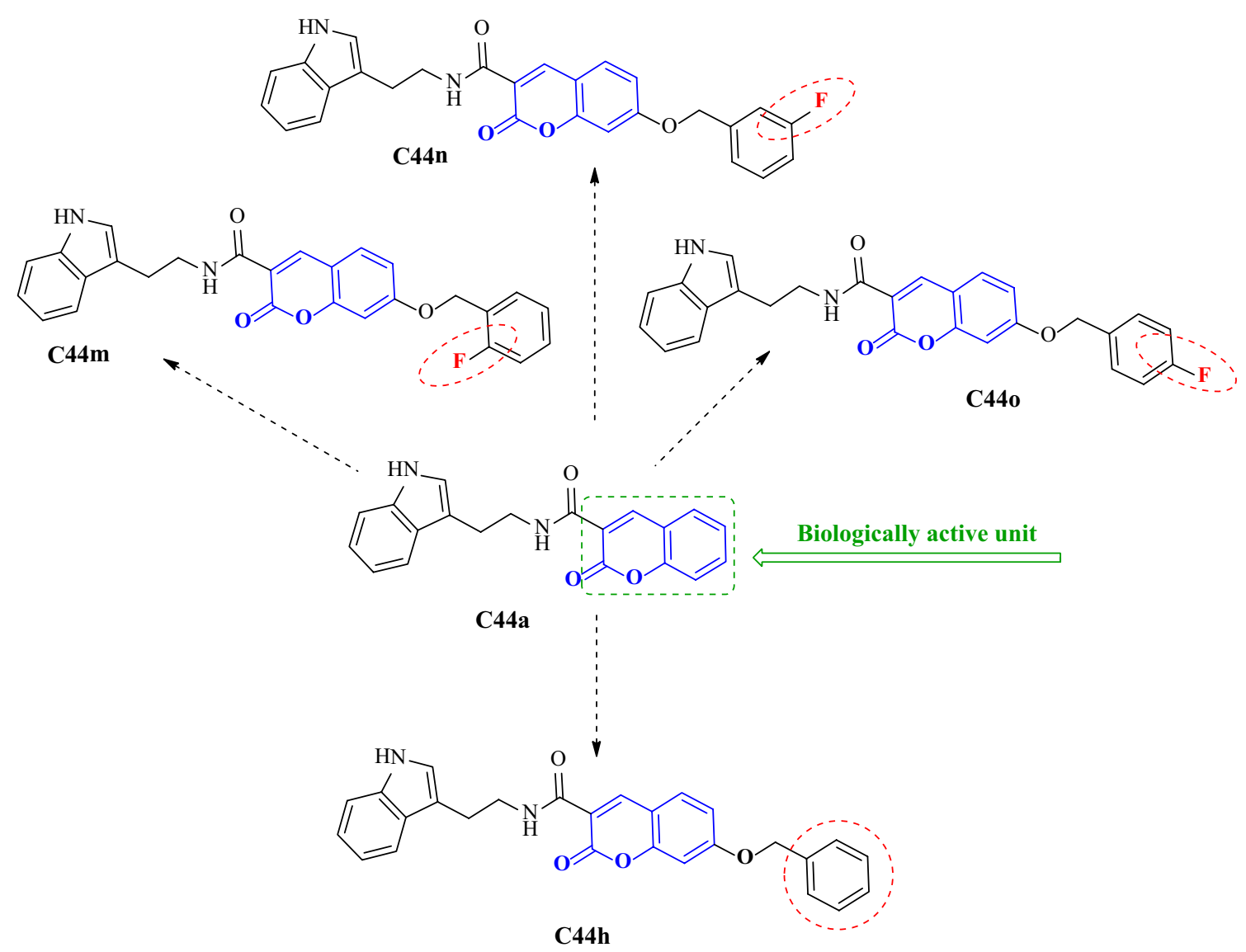

Fig. 44 Comparative structure of coumarin carboxamides 44a, C44m, C44n, C44o and C44h
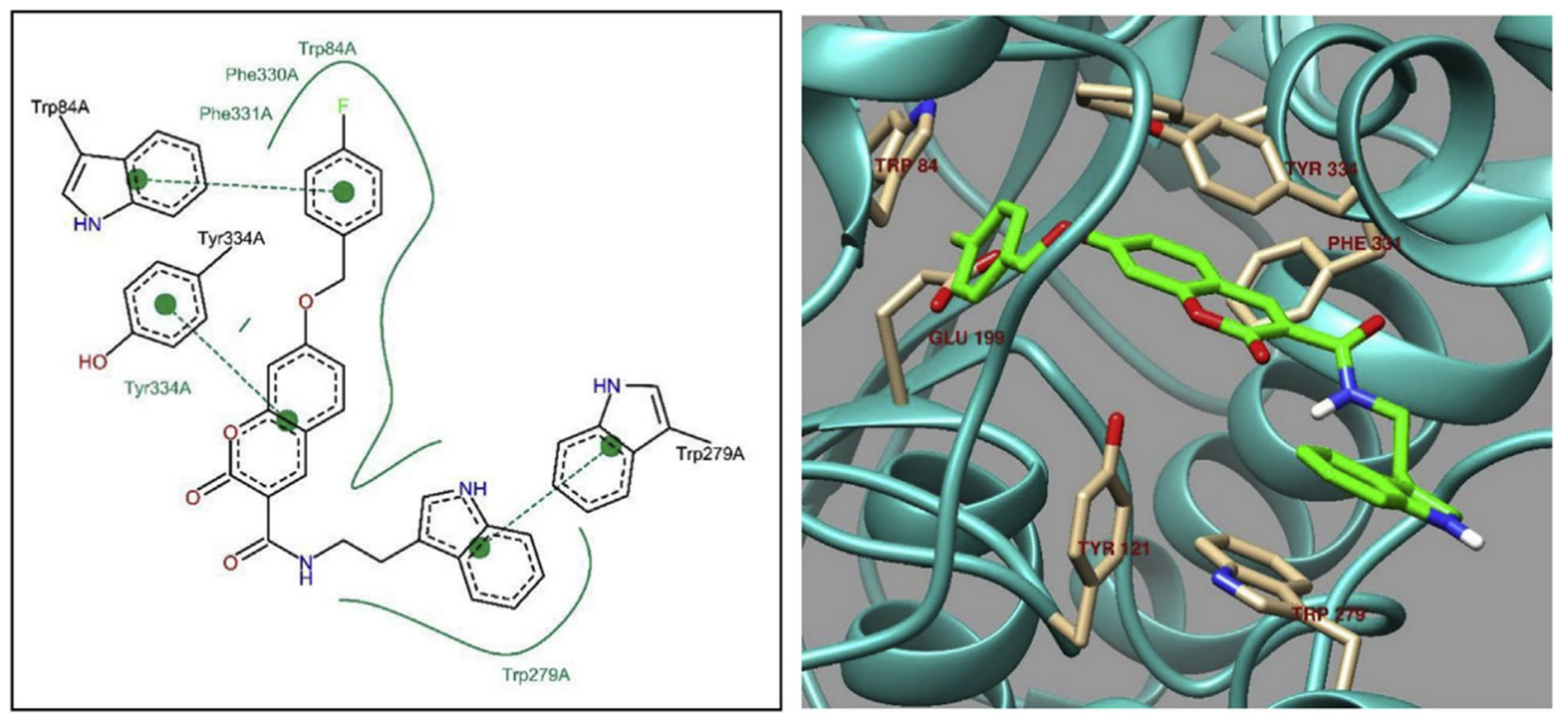

Fig. $452 D$ (left) and 3D (right) representation of interactions of compound C44o in the active site of AChE [53] 


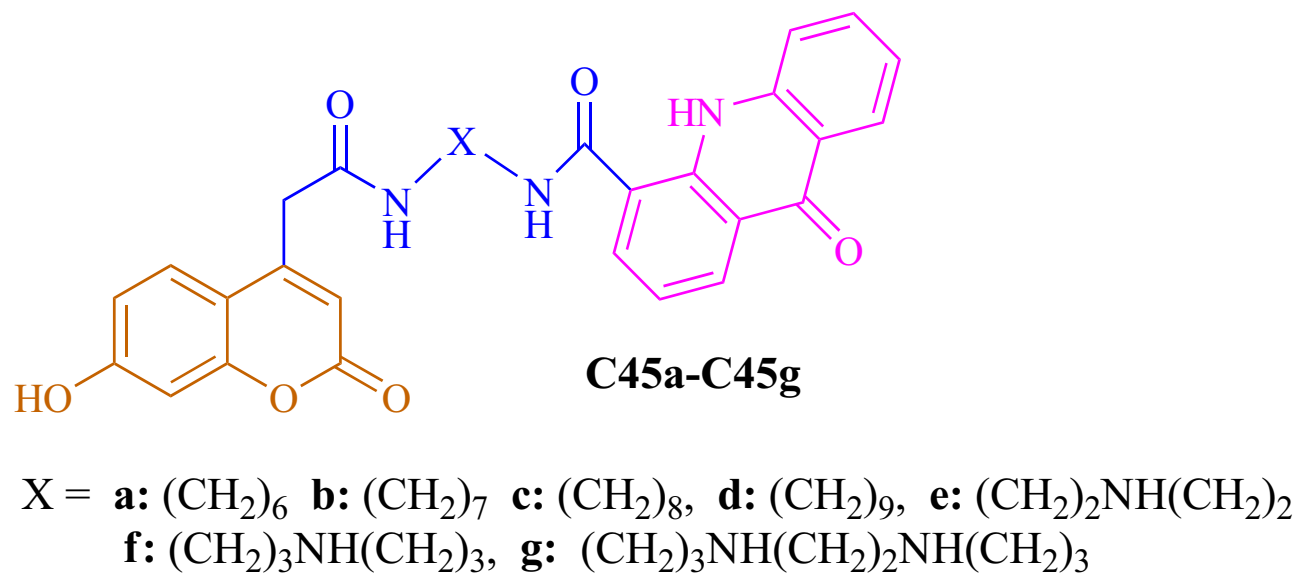

Fig. 46 Structures of synthesized of acridine-coumarin hybrids $\mathbf{C 4 5 a - C 4 5 g}$

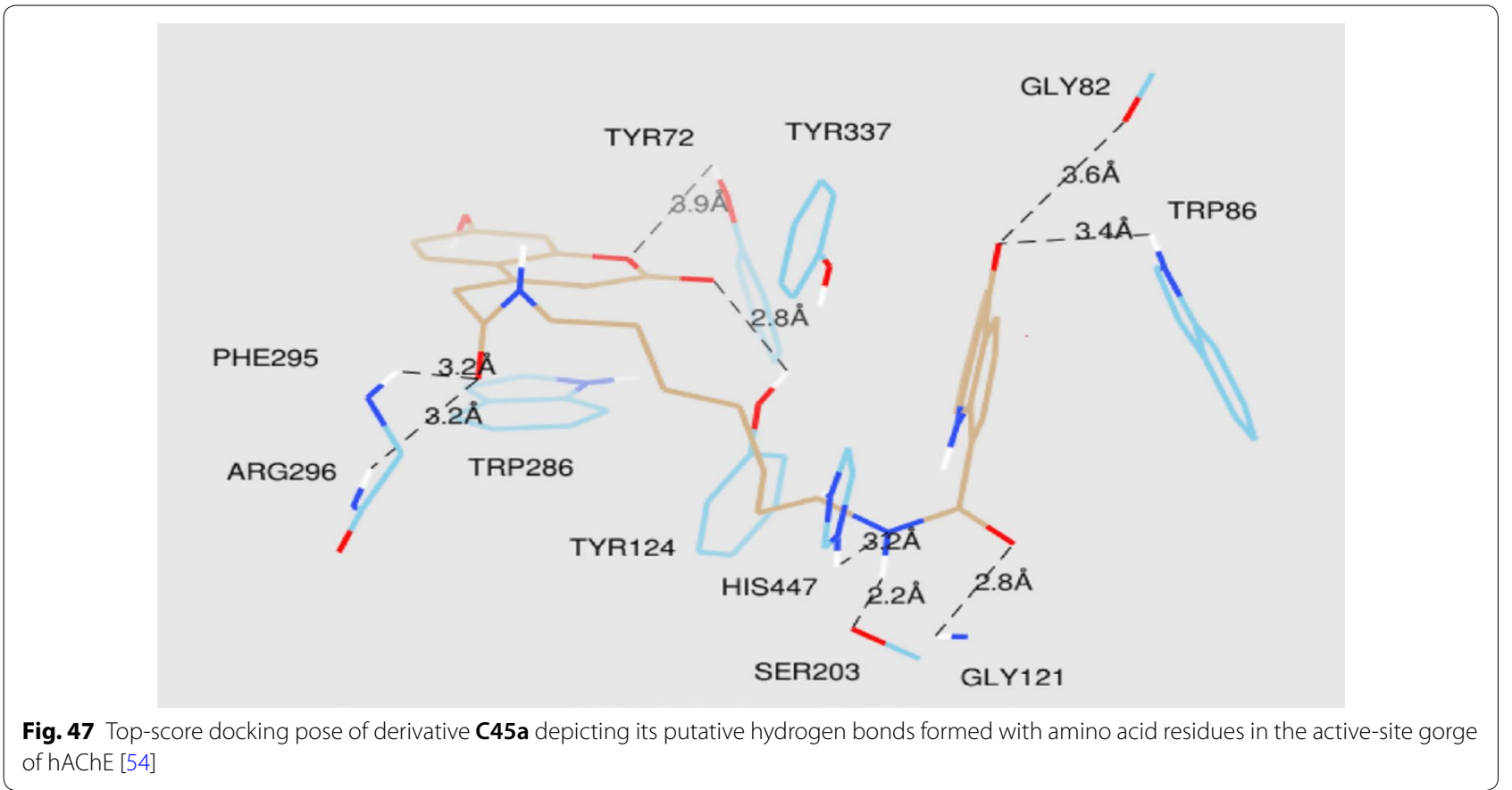

acetylcholinesterase inhibitory activity against human erythrocytes and butyrylcholinesterase inhibitory activity against human plasmatic butyrylcholinesterase against tacrine and the reference drug 7-MEOTA. Among all the compounds tested, C45b with 7 methylene groups exhibited the highest acetylcholinesterase inhibitory activity, with $\mathrm{IC}_{50}$ value of $5.85 \mu \mathrm{M}$ and with potency 3-times stronger when compared to the reference with $\mathrm{IC}_{50}$ of $15 \mu \mathrm{M}$. On the other hand, compound C45c and C45f was found to be most potent against $\mathrm{hBuChE}$ with the $\mathrm{IC}_{50}$ value of $32.53 \mu \mathrm{M}$ and $43.40 \mu \mathrm{M}$ (Fig. 46). Molecular modelling studies were performed to predict the binding modes of compounds C45b, along with C45c and C45f with hAChE/hBuChE.

The lowest energy binding pose of derivative $\mathbf{C 4 5 b}$ with hAChE is depicted in Fig. 47. $\pi-\pi$ binding interactions between coumarin core and the aromatic residue of Trp286 in the PAS of the enzyme was reported. Interaction of Ser302 and His447 of amino acids with the amide group of the acridone core was also reported. Additionally, interaction between amino acids of the active site of the enzyme and ligand was also reported. The direct interaction of compound $\mathbf{C 4 5}$ c with the catalytic triad via His438 andSer198 was clearly reported in the active site of the enzyme hBuChE (Fig. 48). Moreover hydrogen 


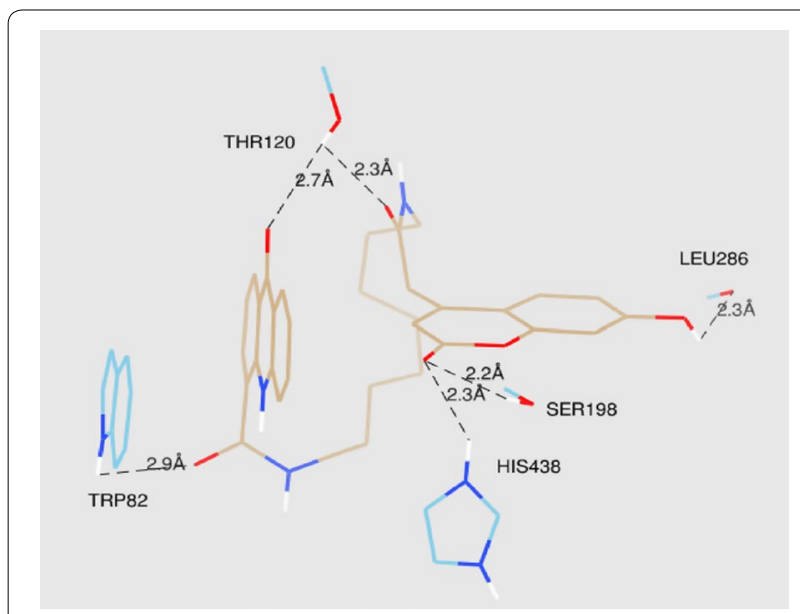

Fig. 48 Top-score docking pose of derivative $\mathbf{C 4 5 b}$ depicting its putative hydrogen bonds formed with amino acid residues in the active-site gorge of hBuChE [54]

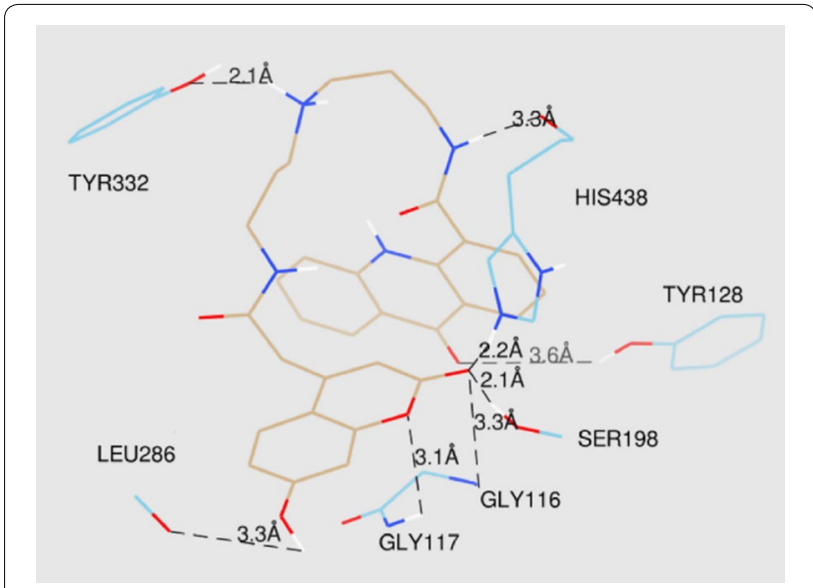

Fig. 49 Top-score docking pose of derivative $\mathbf{C 4 5 f}$ depicting its putative hydrogen bonds formed with amino acid residues in the active-site gorge of hBuChE [54]

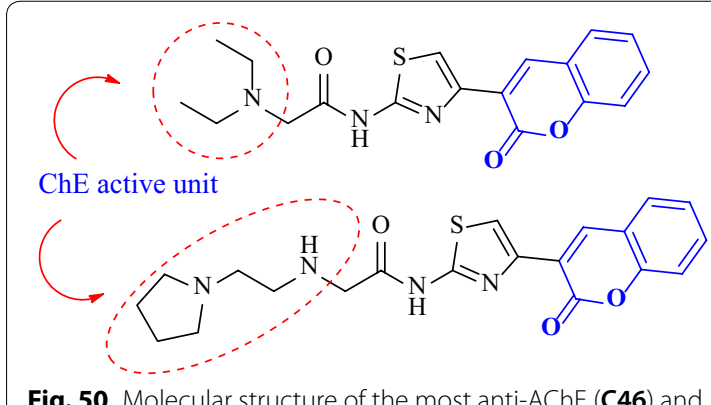
C46 anti-BuChE (C47) thiourea derivatives bonds and $\pi-\pi$ interactions between amino acids of the catalytic cavity and $\mathbf{C 4 5 c}$ was also reported. The pose with the lowest binding energy for derivative C45f with $\mathrm{hBuChE}$ is depicted in Fig. 49. Direct interactions weith the residues His438 andSer198 and one additional intermolecular interaction between the catalytic cavity of the enzyme $\mathrm{BuChE}$ and derivative was also reported. The docking results suggests that acridine seems to be a possible substitute for tacrine in the family of dual binding site inhibitors [54].

Sonmez et al. [55] designed and synthesized coumaryl-thiazole derivatives with the acetamide moiety as a linker between the alkyl chains and/or the heterocycle and tested their potency against AD [55]. Docking studies were performed to check the binding profile of coumarin derivatives into the active site of $\mathrm{AChE}$ and BuChE enzymes against galantamine. Both these cholinesterases (AChE and BuChE) are similar in structure and also $65 \%$ of the amino acid sequences for both of these are similar [56]. The basic difference, is the presence of aromatic amino acid in AChE over BuChE which possess aliphatic amino acids, making them both selective, against different inhibitors of the two enzymes [57]. From all the thiazole derivatives compound C46, 2-(diethylamino)- $\mathrm{N}$-(4-(2-oxo- $2 \mathrm{H}$-chromen-3-yl)thiazol-2-yl)acetamide $\left(\mathrm{IC}_{50}=43 \mathrm{nM}\right)$ was the most potent AChE inhibitor with a selectivity index of 4151.16 over $\mathrm{BuChE}$ and 56-fold more stronger than the standard galantamine $\left(\mathrm{IC}_{50}=2.4 \mu \mathrm{M}\right)$. The BuChE activity of most of the compounds was lesser than their AChE activity except for compound $\mathbf{C 4 7}$ that exhibited the strongest inhibition against BuChE with an $\mathrm{IC}_{50}$ value of $2.35 \mu \mathrm{M}$, which was 2 and 7.5 fold more than the standards donepezil $\left(\mathrm{IC}_{50}=4.66 \mu \mathrm{M}\right)$ and galantamine $\left(\mathrm{IC}_{50}=17.38 \mu \mathrm{M}\right)$ (Fig. 50).

Kinetic enzymatic study was carried out in order to explore the mechanism of inhibition of compound $\mathbf{C 4 6}$ with the enzyme AChE. The Lineweaver-Burk plot (Fig. 51) displayed increased slopes (decreased Vmax) and intercepts (higher $\mathrm{Km}$ ) at higher inhibitor concentration. This pattern indicated a mixed-type inhibition and hence it was concluded that compound $\mathbf{C 4 6}$ was able to bind to CAS, PAS and the catalytic triad of AChE. The inhibition constant $\mathrm{Ki}$, was equal to $31 \mathrm{nM}$ [55].

Yao et al. [58] designed and synthesized coumarin derivatives containing piperazine ring and tested them for their AChE and BuChE inhibitory activity against two standards, huperzine $\mathrm{A}$ and Iso-OMPA. Results suggested that these compounds display significant inhibition for $\mathrm{AChE}$ over $\mathrm{BuChE}$ and compound $N$-(3-chloro-4-((4-ethylpiperazin-1-yl)methyl)phenyl)6-nitro-2-oxo-2H-chromene-3-carboxamide (C48) was reported to be the most potent $\mathrm{AChE}$ inhibitor with $\mathrm{IC}_{50}$ 


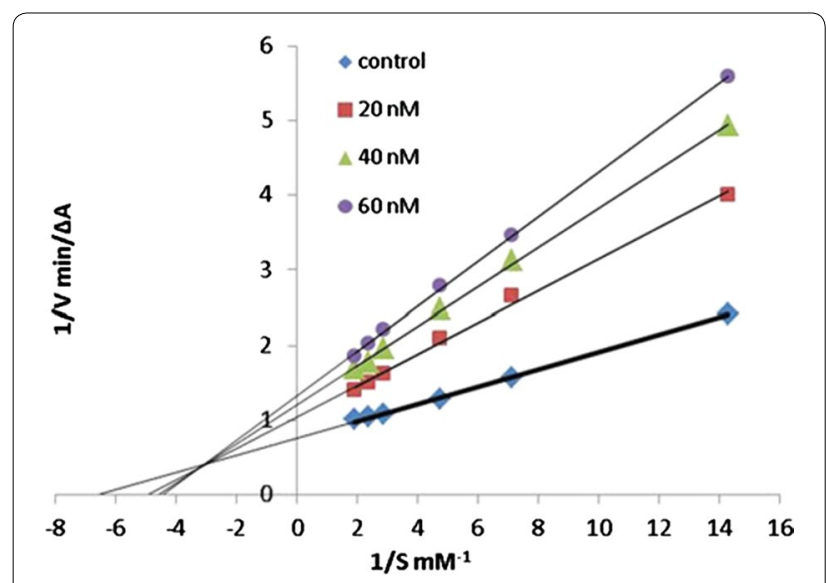

Fig. 51 Kinetic study on the mechanism of AChE inhibition by compound C46. Overlaid Lineweaver-Burk reciprocal plots of AChE initial velocity at increasing substrate concentration $(0.05-0.50 \mathrm{mM})$ in the absence of inhibitor and in the presences of different concentrations of $\mathbf{C 4 6}$ are shown [55]

value of $34 \mathrm{nM}$. The docking results revealed that compound $\mathbf{C 4 8}$ was able to bind to AChE, with PAS of AChE via Trp286 and Arg296 residues and Trp286 was the main residue in ligand recognition as it was found to bind the aromatic rings of coumarin by four $\pi-\pi$ stacking. The $\mathrm{OC}=\mathrm{O}$ group of the coumarin nucleus was reported to interact with Phe295 and Arg296 by two hydrogen bonds. In addition, the central part of amide bond formed hydrogen bond with Phe295 in the deep aromatic narrow gorge. Moreover, the aniline moiety of $\mathbf{C 4 8}$ formed two $\pi-\pi$ stacking with residues Tyr341 and Trp286, whereas its ethylpiperazine moiety covered the CAS via hydrophobic interactions with Phe330 and Trp84, respectively. These results depicted the binding stability of $\mathrm{C} 48$ to AChE. In order to get the insight about the stability of the complex compound C48-AChE, 3 ns MD simulations were successfully performed on compound C48-AChE complex, and the observations indicated well behaved systems (Fig. 52) [58].

\section{Coumarin analogues as MAO inhibitors Introduction to MAO and its sub-isoforms MAO-A} and MAO-B

Monoamine oxidase is a flavin adenine dinucleotide (FAD) containing enzyme, which is tightly bound to the outer membrane of the mitochondria of the neuronal cells, glial cells and to other cells [59]. It works as a catalyst in the oxidative deamination of monoamines either a<smiles>CCN1CCN(Cc2ccc(NC(=O)c3cc4cc([N+](=O)[O-])ccc4oc3=O)cc2Cl)CC1</smiles>

c

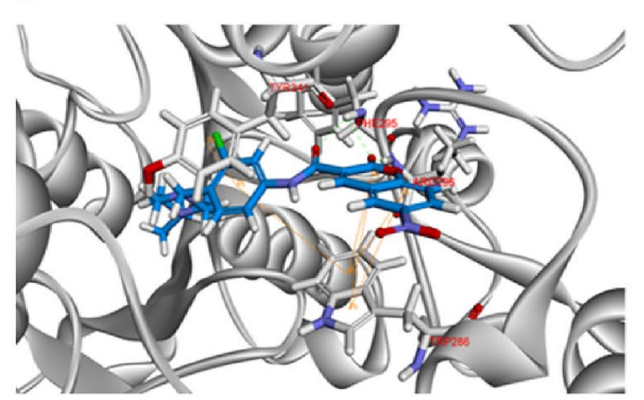

b
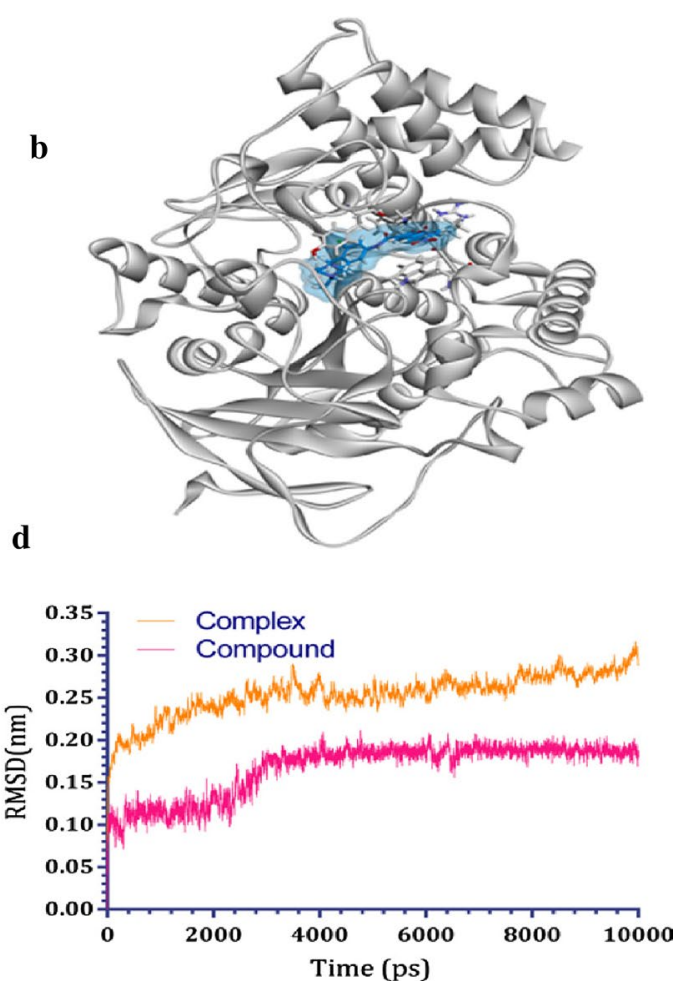

Fig. 52 Molecular modelling, docking and molecular dynamics (MD) simulations of AChE targeting compound C48-AChE. a Molecular structure of compound C48. b Molecular docking of compound C48. c The interactions of compound C48 and active pocket. $\mathbf{d}$ Molecular dynamics of compound C48-AChE complex [58] 
Table 1 Main difference between the two isoforms MAO-A and MAO-B

\begin{tabular}{|c|c|}
\hline MAO-A & MAO-B \\
\hline It preferentially oxidizes nor-epinephrine and serotonin & Preferentially deaminates $\beta$-phenylethylamine and benzyl-amine $[65,66]$ \\
\hline It is selectively inhibited by clorgyline & Selectively inhibited by $\mathbf{L}$-deprenyl $[65,66]$ \\
\hline $\begin{array}{l}\text { Both the MAO isoforms have a different tissue distribution. } \\
\text { Occurs in cathecholaminergic neurons as well as glia }\end{array}$ & $\begin{array}{l}\text { MAO-B is predominant in the human brain, and is compartmentalized into different } \\
\text { cell types. It occurs mainly in glial cells and serotoninergic neurons }[67,68]\end{array}$ \\
\hline $\begin{array}{l}\text { MAO-A inhibitors have been used mostly in the treatment of } \\
\text { mental disorders, in particular depression and anxiety }[80,81]\end{array}$ & Used in the treatment of Parkinson's disease and perhaps, Alzheimer's disease [70, 82] \\
\hline
\end{tabular}<smiles>CC(C)NNC(=O)c1ccncc1</smiles>

Iproniazide (I) (A/B)<smiles>C#CCN(C)[C@@H](C)Cc1ccccc1</smiles>

L-Deprenyl (I) (B)<smiles>C#CCN(C)Cc1ccccc1</smiles>

Pargyline (I) (A/B)<smiles>O=C(NCCN1CCOCC1)c1ccc(Cl)cc1</smiles>

Moclobemide (R) (A)<smiles>COc1cc(Br)c2oc(C3CCNCC3)cc2c1</smiles><smiles>Cc1cccc(N2CC(CO)OC2=O)c1</smiles>

Toloxatone (R) (A)<smiles>CCS(=O)(=O)Oc1ccc2c(C)c(C)c(=O)oc2c1</smiles>

Esuprone (R) (A)<smiles>Cc1c(C)c2ccc(OCc3nnc(C(C)C)s3)cc2oc1=O</smiles>

Fig. 53 Reversible (R) and irreversible (I) MAO-A/B inhibitors

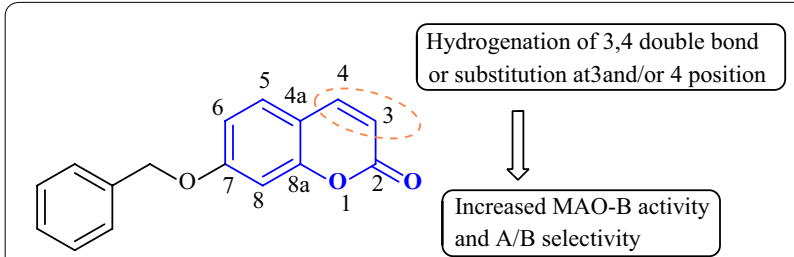

Fig. 54 7-Benzyloxy coumarin C49

from endogenous sources or from exogenous sources. Therefore, it affects the concentrations of neurotransmitter amines as well as many xenobiotic ones [60]. It is also responsible for the biotransformation of a Parkinson producing neurotoxin i.e. 1-methyl-4-phenyl-1,2,3,6tetrahydropyridine (MPTP) into 1-methyl-4-phenylpyridinium [61-63]. It is reported to also inhibit MAO activity-suppressed cell death hence actively participating in the apoptosis process [64]. MAO exists in two isoforms, which are MAO-A and MAO-B which differ from each other in quite a number of factors such as different inhibitor, amino acid sequence, and substrate specificities (Table 1).

As compared to the nonselective-irreversible MAO-A and MAO-B inhibitors (MAO-Is) which were initially used with severe side effects for treating depression [69], the present selective and reversible inhibitors of MAO-A and MAO-B are rather more useful for treating depression, anxiety as well as coadjuvant agents in the treatment of Parkinson's and Alzheimer's disease [70]. Figure 53 depicts the molecular structure of few such nonselectiveirreversible and selective-reversible inhibitors. Like in the category of nonselective-irreversible are iproniazide [71] and pargyline [72], in the selective-reversible category of MAO-A-Is are moclobemide, brofaromine, toloxatone [73-77] and esuprone [78] and in the selective-reversible category of MAO-B-Is is LU 53439 [79]. 
<smiles>O=C(O)c1cc2ccccc2oc1=O</smiles><smiles>Cc1ccc2oc(=O)c(C(=O)O)cc2c1</smiles><smiles>O=C(Cl)c1cc2cc(Cl)ccc2oc1=O</smiles>

Fig. 55 Molecular structure of compound C50, C51 and C52

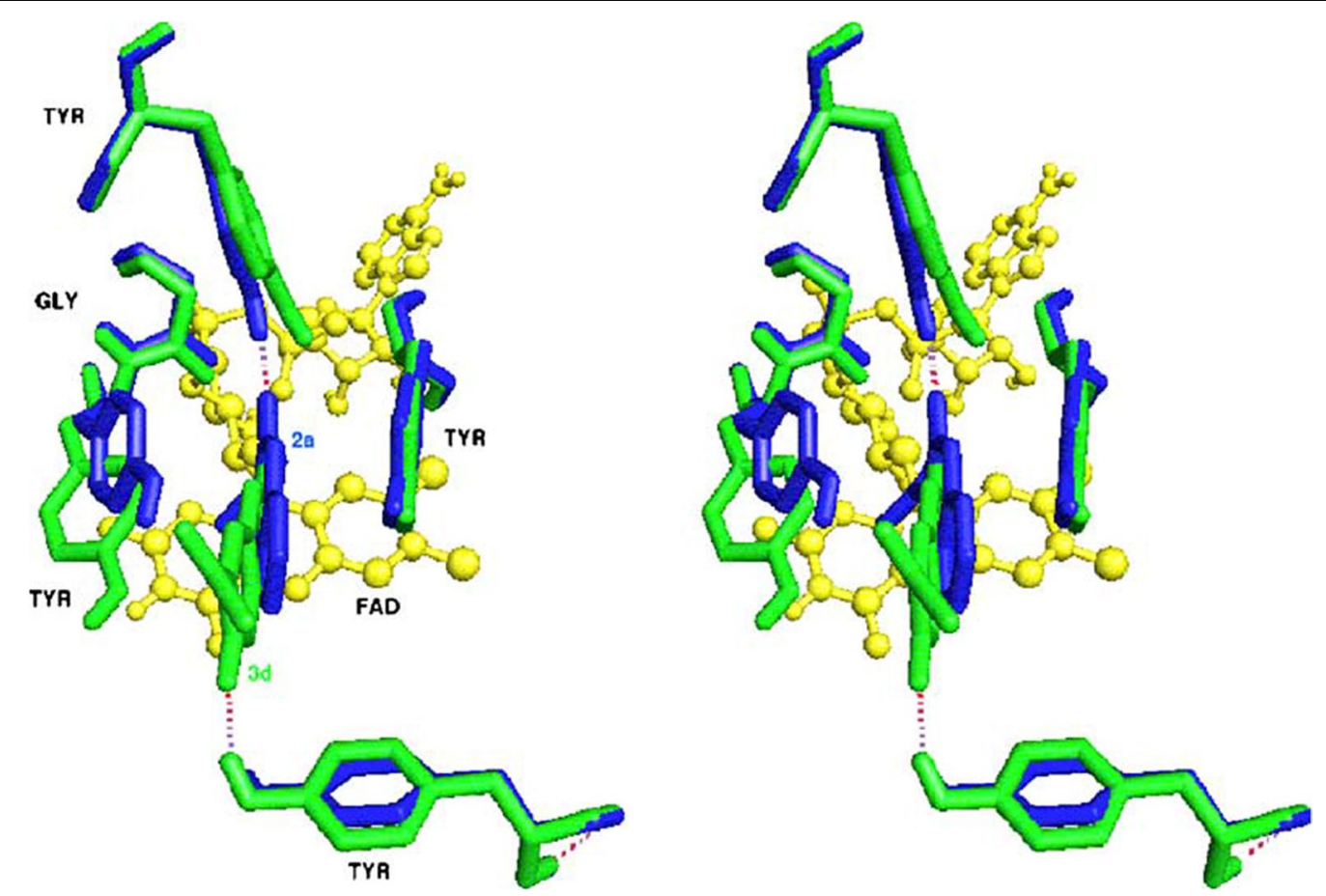

Fig. 56 Stereoview comparison of lowest energy MAO-B complexes with C52 (green polytube model) and C50 (blue polytube model) within the enzymatic cleft. The cofactor is displayed in ball-and-stick style and the hydrogen bond network in dashed lines [88]

The reason behind so many different structure of MAO-Is is because of the fact that the active site of MAOs are still to be discovered. The structure of the active site of MAO-A is still unknown today, therefore there is a limitation in the design of new potent selective MAO inhibitors. Lately in the year 2002, Binda et al. have made an excellent effort in describing the experimental crystal structure of human MAO-B. These group of researchers crystallized the pargyline inhibitor covalently bounded to the N5 atom on the re side of the flavin moiety of the enzyme, identifying the residues bonding the catalytic cavity [83]. A step forward and a recent discovery in 2007 by the same author Binda et al. about the crystal structures of the two isoforms of human MAO were determined, which elucidates the mechanism underlying the selective interactions between these proteins and their ligands, probes the catalytic mechanism [84]. Among the various types of MAO-Is already discovered, the recent interest was diverted towards the $(1 \mathrm{H})$-benzopyran structure and one of the most important example of benzopyran structure is the coumarin nucleus. It has been reported that the hydrogenation of 3,4-double bond and substitution at position 3 and/or 4 of the coumarin ring, modulated the MAO-B inhibitory property and A/B selectivity $[85,86]$. Besides esuprone and LU53439 as discussed earlier (Fig. 53), recently 7-benzyloxy coumarin derivatives (C49) (Fig. 54) are gaining immense attention due to their inhibitory activity and selectivity towards MAO-B [87].

\section{Coumarin analogues as dual MAO-A and MAO-B inhibitors}

Chimenti et al. [88] designed and synthesized coumarin derivatives and studied their role in inhibition of MAOs via computation docking study. It was reported for the coumarin-3-carboxylic acids to be selective-reversible 


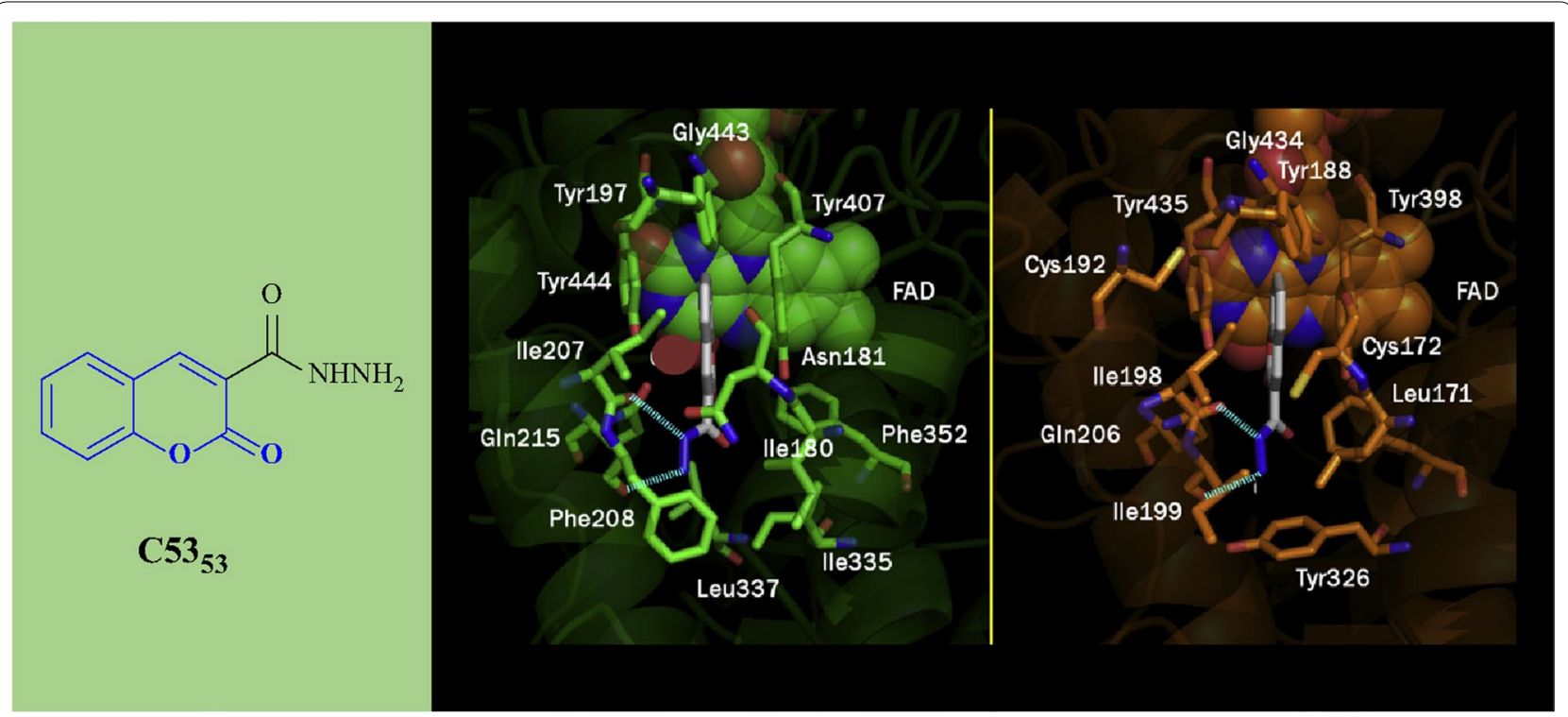

Fig. 57 Most stable binding of $\mathbf{C 5 3}_{\mathbf{5 3}}$ with respect to hMAO-A (left side) and hMAO-B (right side). Ligands are shown in CPK colored sticks, interacting residues are coloured in green (hMAO-A) or orange (hMAO-B) carbons in sticks rendering. FAD is reported in spacefill with green (hMAO-A) or orange (hMAO-B) carbon atoms. Non-interacting aminoacids are in transparent green (hMAO-A) or orange (hMAO-B) cartoon notation. Inhibitor-enzyme hydrogen bonds are reported in cyan dashed lines [89]

Table 2 Comparison between theoretical affinity and experimental inhibition data of $\mathrm{C}_{53} 3_{53}$

\begin{tabular}{llllll}
\hline Compound & \multicolumn{2}{l}{$\mathrm{hMAO-A}$} & & \multicolumn{2}{l}{$\mathrm{hMAO-B}$} \\
\cline { 2 - 3 } \cline { 5 - 6 } & $\mathrm{pIC}_{\mathbf{5 0}}$ & $\Delta \mathrm{G}(\mathrm{kcal} / \mathrm{mol})$ & & $\mathrm{plC}_{\mathbf{5 0}}$ & $\Delta \mathrm{G} \mathrm{(kcal} / \mathrm{mol})$ \\
\hline $\mathrm{C53}_{53}$ & 4.00 & -35.10 & & 8.49 & -38.10 \\
\hline
\end{tabular}

$$
\begin{array}{ll}
\text { C54a; } R_{1}=B r, R_{2}=O M e, R_{3}=H & \text { C55a; } R_{1}=B r, R_{2}=O M e, R_{3}=H \\
\text { C54b; } R_{1}=B r, R 2=R_{3}=O M e & \text { C55b; } R_{1}=B r, R 2=R_{3}=O M e \\
\text { C54c; } R_{1}=B r, R_{2}=O H, R_{3}=H & \text { C55c; } R_{1}=B r, R_{2}=O H, R_{3}=H \\
\text { C54d; } R_{1}=B r, R_{2}=R_{3}=O M e & \mathbf{C 5 5 d} ; R_{1}=B r, R_{2}=R_{3}=O M e
\end{array}
$$

Fig. 58 Molecular structure of compounds C54a-d and C55a-d

inhibitors of isoform MAO-B. Specifically compound C50 was the most potent with $\mathrm{IC}_{50}$ value of 7.76 and selectivity index of 2.94 followed by compound $\mathbf{C 5 1}$ with $\mathrm{IC}_{50}$ of 7.72 and SI of 2.80. For the 3-acyl chlorides coumarin derivatives, it was reported to be active, against both the isoforms MAO-A and MAO-B with compound C52 being the strongest against $\mathrm{MAO}-\mathrm{B}$ with $\mathrm{IC}_{50}$ value of 8.0 (Fig. 55).
In order to rationalise the activity as well the selectivity and get the insight information related to the enzyme and inhibitor interaction docking was performed with the two most active compounds $\mathbf{C 5 0}$ and $\mathbf{C 5 2}$ with the sub isotype MAO-B. It was reported that both these compounds easily fit into the active site of MAO-B with quite different binding modes. The acid moiety of C50 was reported to direct towards the cofactor and the corresponding acid chloride moiety in compound $\mathbf{C 5 2}$, was found to be in the direction opposite protruding towards Tyr326. In addition, both these compounds were found to make one hydrogen bond interaction. For the case of C50, the H-bond was established with the phenolic- $\mathrm{OH}$ of Tyr188 and for C52 with the phenolic-OH of Tyr326. On substituting the carboxylic-OH group with chlorine, it was found that compound $\mathbf{C 5 0}$ was establishing one H-bond with the phenolic-OH of Tyr188, and C52 was reported to establish another $\mathrm{H}$-bond with the phenolic-OH of Tyr326. The difference in this behaviour was justified by the stronger steric and electrostatic repulsion between the acyl chloride moiety and Gly434. The presence of chlorine at position- 6 in the complex, which was actually in close contact with residues Gly434 and tyr188, induces the coumarin moiety of compound $\mathbf{C 5 2}$ to move far from these two residues resulting in different binding modes for the acid and chloride compounds. Therefore, the main difference between these two binding modes is because of the H-bond acceptor contributions of the hydroxyl side chain moieties of Tyr326 and 
<smiles>CCOC(=O)COc1ccc2cc(-c3ccc(C)cc3)c(=O)oc2c1</smiles>

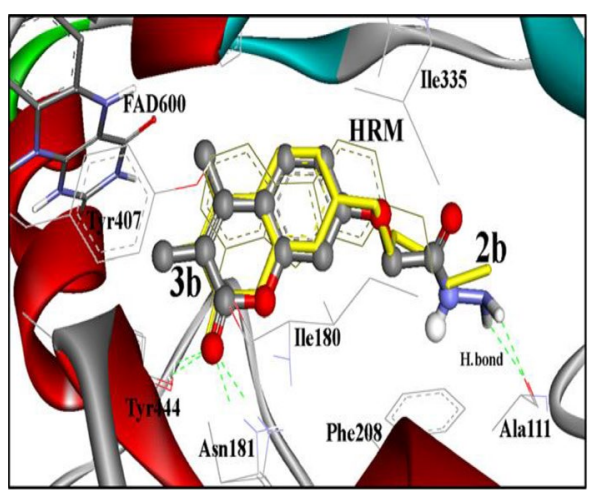

C57<smiles>Cc1ccc(-c2cc3ccc(OCC(=O)NN4C(=O)CCC4=O)cc3oc2=O)cc1</smiles>

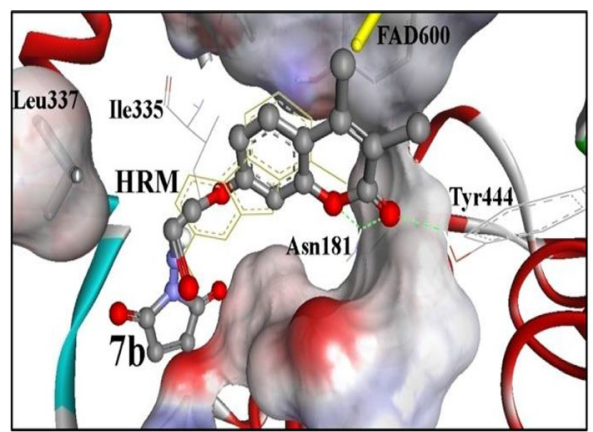

Fig. 59 Molecular structures and binding affinities of compound C56 (yellow stick) and C57 (ball and stick, coloured by element) and C58 into binding pocket of MAO-A (2Z5X) [91]

Tyr188, which can only establish productive interactions with coumarin derivatives (Fig. 56) [88].

Secci et al. [89] designed and synthesized a big library of 3-carbonyl $\left(\mathbf{C 5 3}_{1}-\mathbf{C 5 3 _ { 2 6 }}\right)$, 3-acyl $\left(\mathbf{C 5 3}_{27}-\mathbf{C 5 3 _ { 5 2 }}\right)$, and 3-carboxyhydrazido $\left(\mathbf{C 5 3}_{53}-\mathbf{C 5 3}_{58}\right)$ coumarins derivatives and tested them in vitro for their human monoamine oxidase A and B (hMAO-A and hMAO-B) inhibitory activity. They performed a detailed study related to the position of different substituents on the coumarin ring, that in which way or the other they are effecting the MAO inhibitory activity and selectivity. They concluded from their observation that introduction of hydrazido moiety at position-3 $\left(\mathbf{C 5 3}_{\mathbf{5 3}}\right)$ and substitution at position-7 of 3-ethylester coumarin ring was enhancing the $\mathrm{IC}_{50}$ value from $\mu \mathrm{M}$ to the $\mathrm{nM}$ range $\left(\mathrm{IC}_{50}=3.22 \mathrm{nM}\right)$ with respect to the reference drug $\mathrm{R}-(-)$-deprenyl and also displaying selectively for the hMAO-B isoform. Hence to get the insight information for the most potent compound of the library viz $\mathbf{C 5 3}_{\mathbf{5 3}}$, molecular docking was performed against $\mathrm{MAO}-\mathrm{A}$ and $\mathrm{MAO}-\mathrm{B}$ taking in consideration the most stable binding configuration of $\mathbf{C 5 3}_{\mathbf{5 3}}$ (Fig. 57). Coumarin moiety was reported to be into the binding cleft of hMAOs and was found to form $2 \mathrm{H}$-bonds with the targets and $\pi-\pi$ stacking interactions with Tyr407 and Tyr444 of hMAO-A and with Tyr435 and Tyr398 of hMAO-B. Table 2 displays that the $\Delta G$ of binding was in good correlation $\left(\mathrm{r}^{2}=0.76\right)$ to the inhibitory activities for compound $\mathbf{C 5 3}_{\mathbf{5 3}}$ [89].

Matos et al. [90] designed and synthesized two different series of 3-phenylcoumarins (C54a-C54d) and 3-benzoylcoumarins (C55a-C55d) (Fig. 58). Bromo atom was introduced at position- 6 of the coumarin ring whereas $\mathrm{OCH}_{3} / \mathrm{OH}$ group was introduced at position-8 and then these compounds were evaluated for their MAO-A and MAO-B inhibitory activities using R-(-)deprenyl and iproniazide as standards. Interestingly, the presence and absence of the carbonyl moiety between the coumarin ring and 3-phenyl ring was focused for the MAO-A/MAO-B inhibitory properties. The 3-phenylcoumarin derivatives, C54a $\left(\mathrm{IC}_{50}=83.48 \mathrm{nM}\right)$ with bromo at position 6 and methoxy at position 8 and derivative C54b $\left(\mathrm{IC}_{50}=1.35 \mathrm{nM}\right)$ with bromo at position 6, methoxy at position 8 and another methoxy at the para position of the 3-phenyl ring were found to be the most active compounds among the eight. To be added, it was reported that the introduction of a substituent in the para position of the 3-phenyl ring might had helped in improved activity, specifically when it is a methoxy 
a
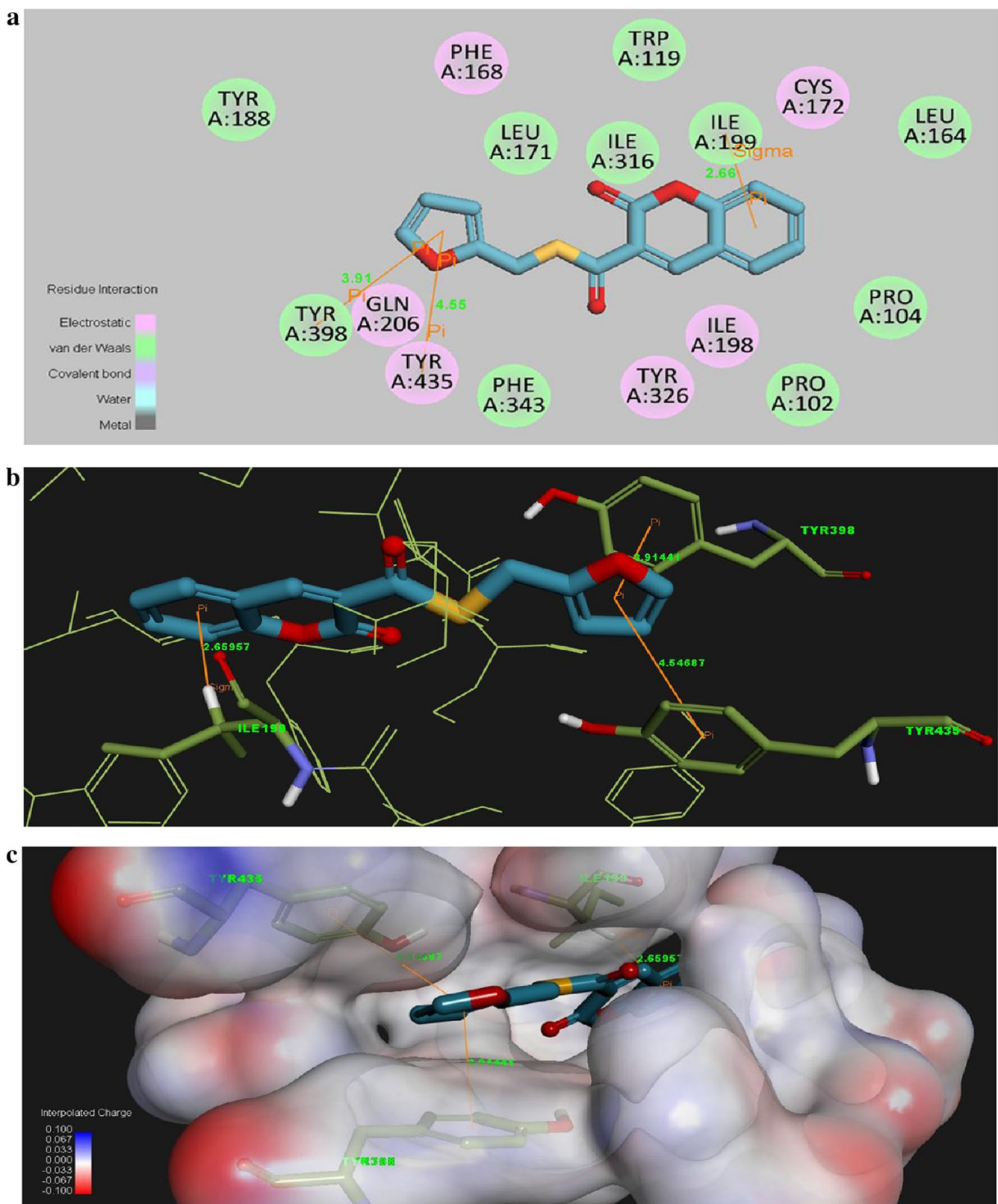

Fig. 60 Molecule docking of the compound C59 into the ligand binding model site of MAO-B (a). 2D Picture of binding (b). 3D Picture of binding (c). Molecule well fitted in the active pocket at the enzyme surface [92]<smiles>O=c1cc(-c2ccccc2O)oc2ccccc12</smiles>

Fig. 61 Molecule structures of compounds $\mathbf{C 6 0}$ and $\mathbf{C 6 1}$ group (C54b). This derivative was found displaying higher MAO-B activity even better than the standard $\mathrm{R}$-(-)-deprenyl $\left(\mathrm{IC}_{50}=19.60 \mathrm{nM}\right)$ with the inhibition level more than 74,074-fold with respect to its MAO-A isoform and as compared to other derivatives. On the other hand, the presence of hydroxyl group in the places of methoxy group was however found lowering the activity and selectivity as reported for the derivatives C54c $\left(\mathrm{IC}_{50}=30.91 \mathrm{nM}\right)$ and C54d $\left(\mathrm{IC}_{50}=16.87 \mathrm{nM}\right)$. On 


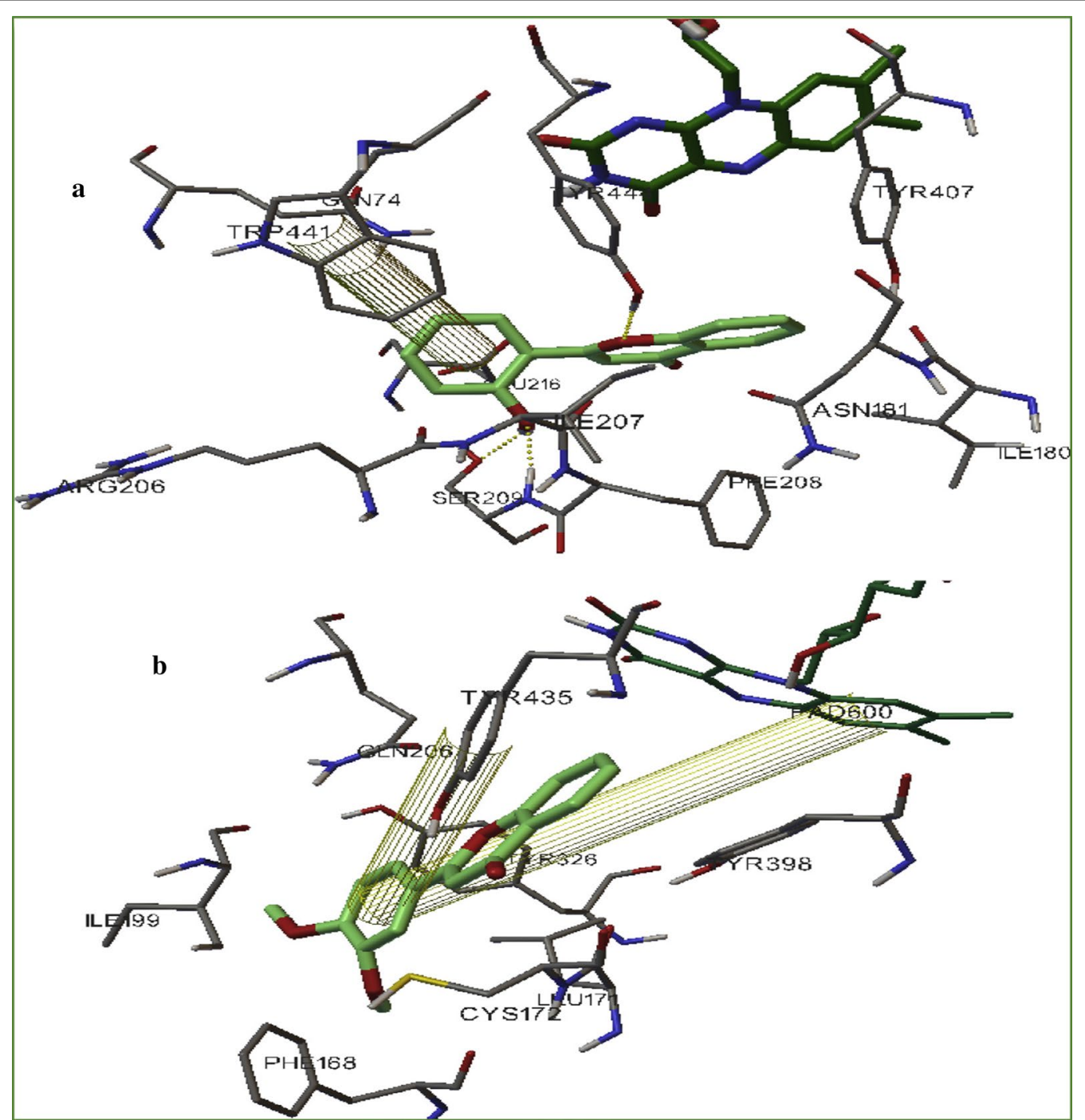

Fig. 62 a Interaction of compound C61 with human MAO-A. b Interaction of compound C60 with human MAO-B [93]

the comparison of these results with the other series of 3-benzoylcoumarins where the 3-phenyl group was replaced by the 3-benzoyl group, it was reported that the carbonyl moiety was selectively lowering the MAO-B activity as compared to MAO-A activity and the structure activity relationship was just the opposite as found for the 3-phenylcoumarins. In here, the 6-bromo-8-hydroxy derivatives (C55c and C55d) $\left(\mathrm{IC}_{50}=46.81 \mathrm{nM}\right.$ and $19.17 \mathrm{nM}$ ) were reported to be more MAO-A active than their corresponding 6-bromo-8-methoxy forms (compounds $\mathbf{C 5 5}$ a and $\mathbf{C 5 5 b}$ ), which do not displayed any of the MAO inhibitory properties [90].

Abdelhafez et al. [83] designed and synthesized 7-oxycoumarin derivatives and evaluated them in vivo and in vitro for their MAO-A and MAO-B inhibitory properties. The in vitro studies provided very good results with Ki values in the range of picomolar in the terms of potency and MAO-A selectivity, whereas in vivo studies provided with better direct correlation. Overall, compounds C56, C57 and $\mathbf{C 5 8}$ were reported to be the most potent in vitro and in vivo MAO-A and MAO-B inhibitors. In order to get the insight of the binding nature of the compounds, molecular docking was performed by docking these derivatives into the binding sites of MAO-A (PDB: 2Z5X) and MAO-B (PDB: 2XFN). The aforementioned docking results of the biologically potent derivatives C56, C57 and C58, displayed the best fitting into the binding site of MAO-A as compared to the other derivatives. Compound $\mathbf{C 5 6}$ was found to exhibit two $\mathrm{H}$-bonds between its $2-\mathrm{C}=\mathrm{O}$ and $\mathrm{OH}$ of Tyr444 and NH of Asn181 amino acid within RMSD of $0.66 \AA$ A. Compound C57 was reported to tightly bound 
with four $\mathrm{H}$-bonds through the $2-\mathrm{C}=\mathrm{O}$ terminal and $\mathrm{NH}_{2}$ group and $\mathrm{OH}$ of Tyr444, $\mathrm{NH}$ of $\mathrm{Asn} 181$ and $\mathrm{C}=\mathrm{O}$ of Ala111 within RMSD of $1.51 \AA$. Analogue $\mathbf{C 5 8}$ was reported to display very good binding affinity and was found to tightly bound with three $\mathrm{H}$-bonds between the $\mathrm{O}$ (pyrone) and 2- $\mathrm{C}=\mathrm{O}$, with $\mathrm{NH}$ of $\mathrm{Asn} 181$ and $\mathrm{OH}$ of Tyr444, within RMSD of $1.01 \AA$ A (Fig. 59) [91].

He et al. [92] designed and synthesized two new series of coumarin derivatives viz $2 \mathrm{H}$-chromene-3-carboxamides and $\mathrm{S}-2 \mathrm{H}$-chromene-3-carbothioates as hMAO inhibitors and performed their 3D docking studies to get the better assess to their selectivity. Among all the derivatives, analogue $\mathbf{C 5 9}$ was reported to be the most active $\left(\mathrm{IC}_{50}=0.21 \mu \mathrm{M}\right)$ and 189.2 folds more MAO-B selective with respect to the MAO-isoform and as compared to the standard iproniazid, with $\mathrm{IC}_{50}$ of $7.65 \mu \mathrm{M}$ and selectivity of 1 -fold. Its docking study revealed that the $\mathrm{H}$-atom of the amino acid residue Ile199 was responsible for the

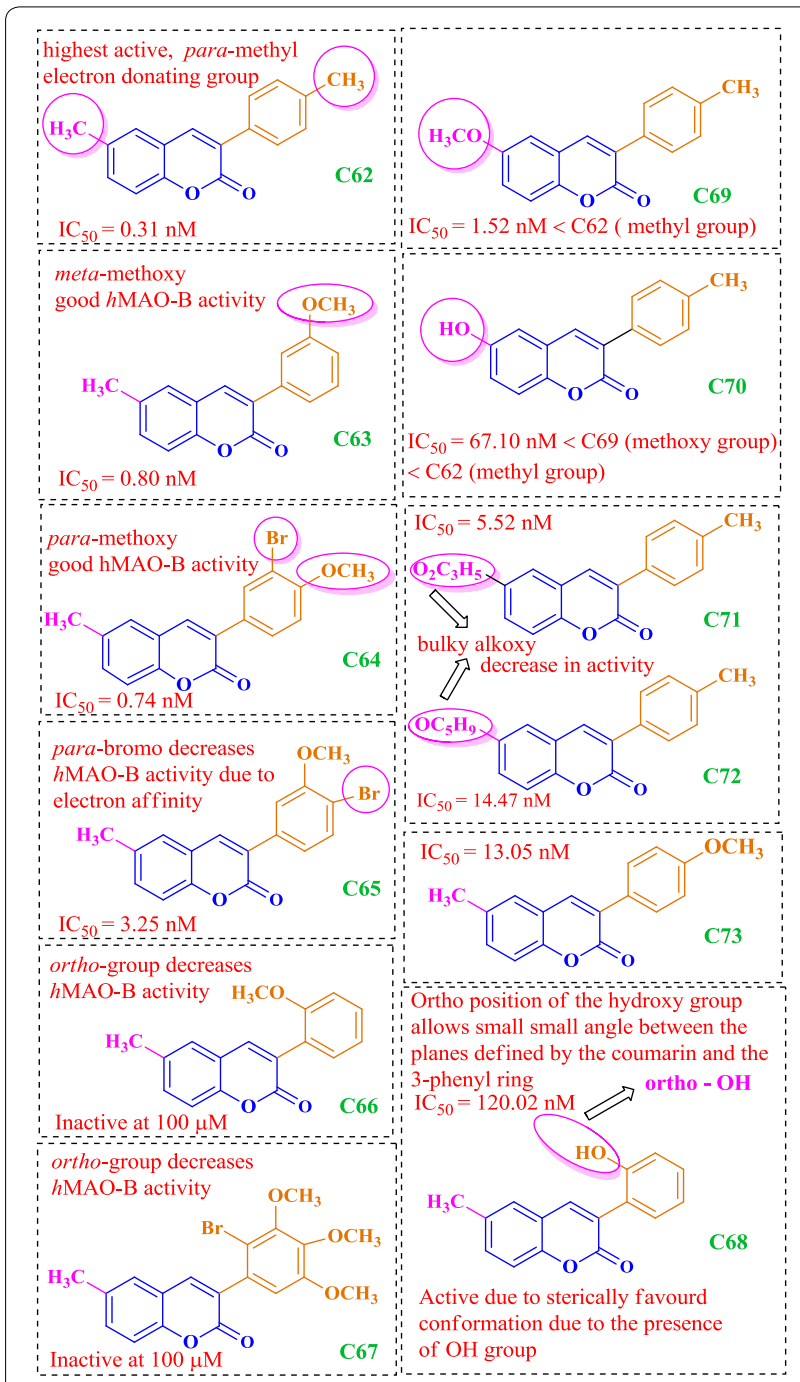

Fig. 63 Molecular structures of compounds C62-C73 binding of ligand's benzene ring through the sigma- $\pi$ interaction. Additionally two $\pi-\pi$ interactions were also reported between the compound and the amino acid around it. One was between the furan-ring of compound C59 and benzene-ring of Tyr398 and the other between the furan-ring of compound C59 and benzene-ring of Tyr435 (Fig. 60) [92].

Nayak et al. [93] designed a large library of twenty 2-aryl-4H-chromen-4-ones coumarin derivatives and performed their hMAO inhibitory activity using recombinant hMAO-A and hMAO-B against selegiline as standard. Result showed that sixteen compounds were selective towards $\mathrm{hMAO}-\mathrm{B}$, and among them compound C60 which was carrying 3,4-di- $\mathrm{OMeC}_{6} \mathrm{H}_{3}$ group was found to be the most active $\left(\mathrm{IC}_{50}=0.16 \mu \mathrm{M}\right)$ and also the most selective $(\mathrm{SI}=30.0) \mathrm{MAO}-\mathrm{B}$ inhibitor. With respect to this, compound $\mathbf{C 6 1}$ carrying the $2-\mathrm{OH}-\mathrm{C}_{6} \mathrm{H}_{4}$ group was found to be the most active $\left(\mathrm{IC}_{50}=0.52 \mu \mathrm{M}\right)$ and the most selective $(\mathrm{SI}=11.5) \mathrm{MAO}-\mathrm{A}$ inhibitor (Fig. 61). The reason behind the potent hMAO-B inhibition property and selectivity of the sixteen derivatives was due to the presence of the deactivating functional groups at para position of the phenyl ring as compared to the compounds with unsubstituted phenyl ring. The more the deactivating nature of the substituent present on the phenyl ring, the more was the recorded potency $\left(\mathrm{NO}_{2}>\mathrm{Br}>\mathrm{Cl}>\mathrm{CN}\right)$ due to less difference in interaction with the MAO-B protein. However, the selectivity criteria for MAO-B was recorded to follow the reverse order. Additionally the compounds possessing meta positioned activating substituents were also reported to exhibit good MAO-B but for sure not better than the derivatives possessing para positioned deactivating functional groups. Molecular docking performed on the most active compound $\mathbf{C 6 0}$ displayed that the phenyl ring bearing 2 $\mathrm{OCH}_{3}$ groups, attached to the position- 2 of the chromenone was accommodated within the hydrophobic tunnel leading to solvent exposed entrance whereas the ring of the chromenone was accommodated in the cage composed by FAD, Tyr398 and Tyr435. A $\pi-\pi$ interaction between the phenyl ring oriented at position- 2 of chromenone and Tyr435 and FAD was also observed. Docking study of the most active MAO-A inhibitor C61 revealed three $\mathrm{H}$-bonding interaction viz., (i) hydroxyl hydrogen of C61 with side-chain hydroxyl oxygen of Ser209, (ii) hydroxyl oxygen of $\mathbf{C 6 1}$ with backbone amino hydrogen of Ser209 and (iii) chromenone ring oxygen with hydroxyl hydrogen of Tyr444. A $\pi-\pi$ interaction between o-hydroxy phenyl group at 2nd position of chromenone and Trp441 was also obswerved. All these interactions kept pocket1 (Aromatic cage: FAD, Tyr407, Tyr444) unoccupied, pocket 3 (Ile180, Ile335, Leu337, Met350, Phe352) partially occupied by chromenone 


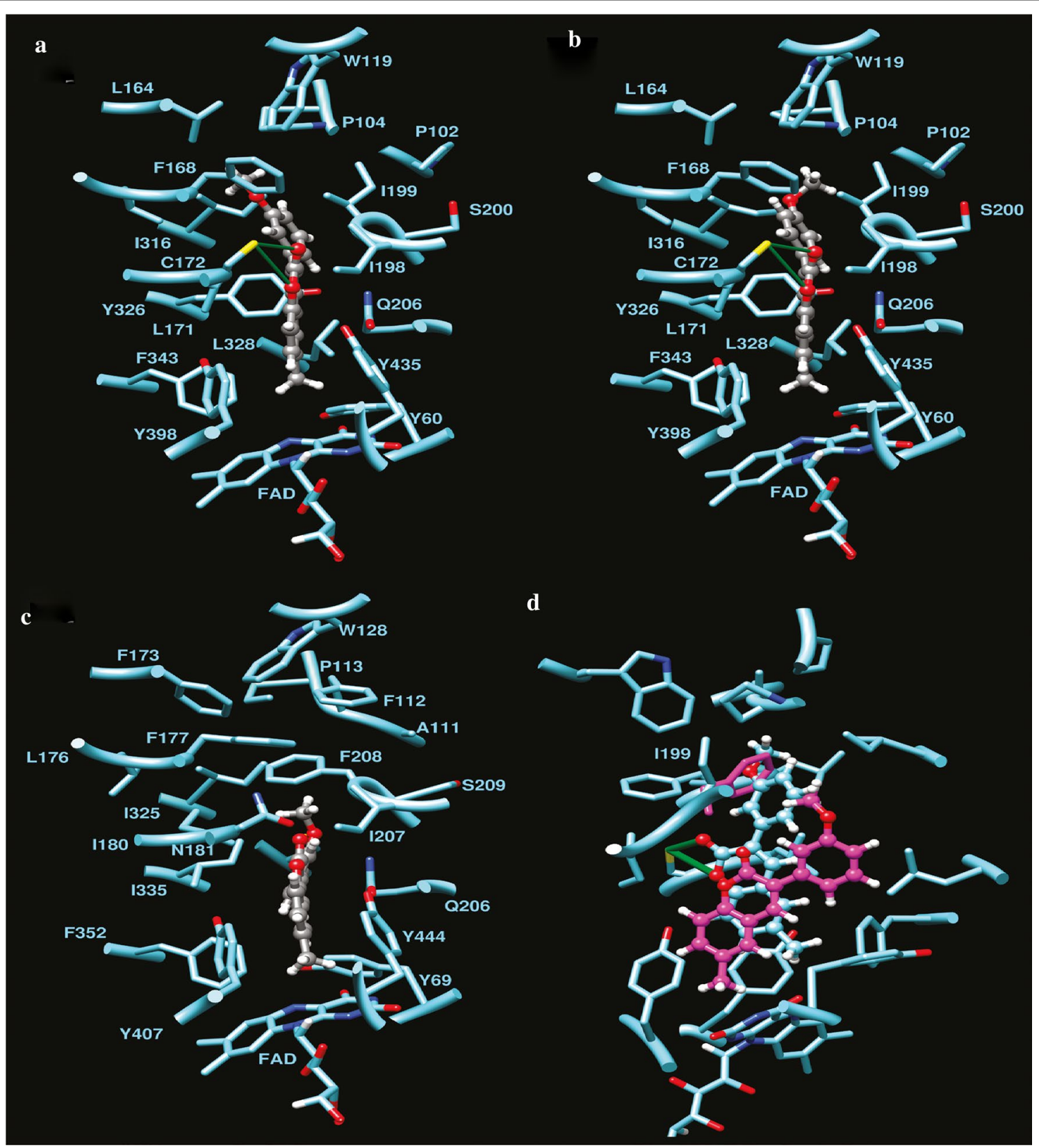

Fig. 64 Molecular docking studies of coumarin derivatives C73 and C63. Most stable binding poses of compound C63 (a) and C73 (b) into MAO-B binding site, and compound $\mathbf{C 6 3}$ (c) into MAO-A binding site. Compounds are colored by atom type, and predicted $\mathrm{H}$-bonds are represented as green pseudobonds. Superposition of binding poses of compound C63 (d) to both isoenzymes, MAO-A (ligand and Phe208 in magenta), and MAO-B [94]

benzene ring and pocket 2 (Gly71, Gln74, Arg206, Ile207, Phe208, Glu216, Trp441) partially occupied by o-hydroxy phenyl ring at 2nd position of chromenone (Fig. 62) [93].

\section{Coumarin analogues as selective MAO-B inhibitors}

Matos et al. [94] synthesized 22 derivatives of 3-arylcoumarins with various alkyl, hydroxyl, halogen, and alkoxy groups in the two benzene rings and check their potential against hMAO-A and hMAO-B. The results showed these compounds to be selective against isoform hMAO$B$ isoenzyme, displaying very good IC50 values in the range of nanomolar and picomolar. Compound C62, was the most potent compound of the series, even 64 times stronger than the drug selegiline and displaying highest 


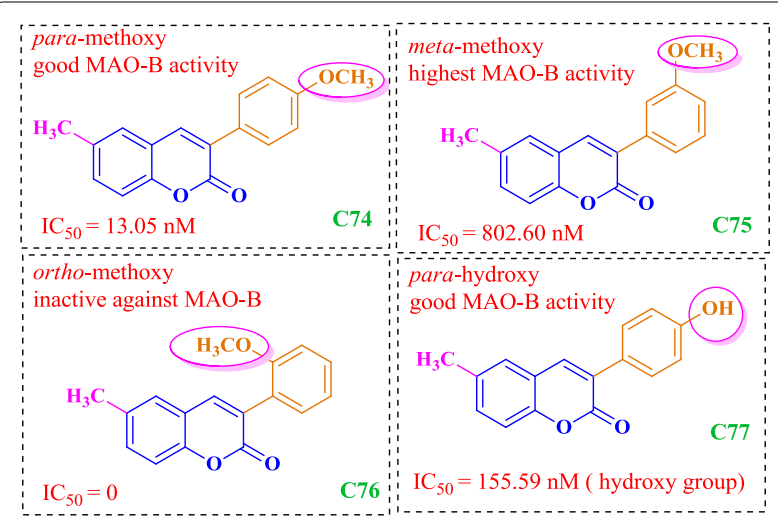

Fig. 65 Molecular structures of the compounds $\mathbf{C 7 4 - C 7 7}$

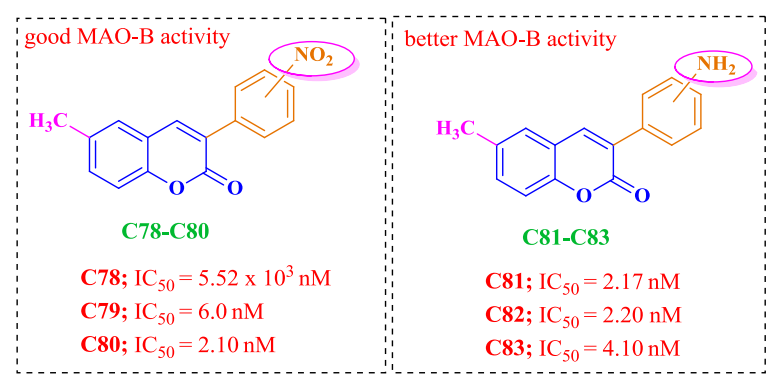

Fig. 66 Molecular structures of the compounds $\mathbf{C 7 8 - C 8 3}$

$\mathrm{IC}_{50}$ of $0.31 \mathrm{nM}$ against hMAO-B, followed by compound $\mathbf{C 6 3}$ and $\mathbf{C 6 4}$, displaying $\mathrm{IC}_{50}$ against $\mathrm{hMAO}-\mathrm{B}$ of $0.80 \mathrm{nM}$, and $0.74 \mathrm{nM}$, respectively. It was found that modification with the electron donating group at para position of the benzene ring helped in increasing the selectivity towards the isoform $\mathrm{B}$ as reported for derivative $\mathbf{C 6 2}$ possessing a p-methyl moiety in the 3-phenyl ring and a 6-methyl moiety in the aromatic coumarin ring as compared to the compound $\mathbf{C 6 5}$, with a parabromo and meta-methoxy groups, respectively. Presence of electron withdrawing group at para in its case was reported to diminish the activity. Compound $\mathbf{C 6 3}$ possessed a meta-methoxy and compound $\mathbf{C 6 4}$ possessed a para-methoxy and a meta-bromo group, respectively in the 3-phenyl ring and a 6-methyl moiety in the aromatic coumarin ring. Additionally, it was found that almost any substituent at ortho position in the 3-phenyl ring tends to decrease the activity as observed for the cases $\mathbf{C 6 6}$ and C67 with an ortho-methoxy and an ortho-bromo except for the compound $\mathbf{C 6 8}$ with an ortho-hydroxy group, which was further explained by docking studies. Moreover, the presence of 6-methyl group in the coumarin moiety tend to increase the activity ( $\mathbf{C 6 2}$ ) as compared to the presence of methoxy (C69) or hydroxyl groups (C70). Also, bulkier alkoxy groups at 6-position such as, 2-oxopropoxy (C71) or a cyclopentyloxy (C72), significantly reduced the activity (Fig. 63) [94].

All these results were found to be consistent with the docking results with the observation that small substituents are more favourable at sixth position of the coumarin nucleus. The bulky substituents resulted in the disruption of the proposed binding mode by occupying most of the space and hence resulting in the loss of the key interactions necessary for the stabilization of the ligand-enzyme complex (C71 and C72). Same ways a small angle between the planes of coumarin and 3-phenyl

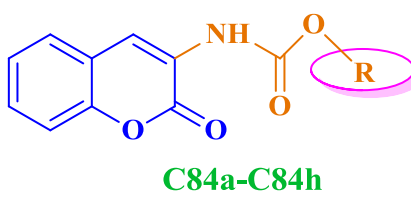
a : $\mathrm{R}=\mathrm{CH}_{3}$;
$\mathrm{IC}_{50}=0$
b : $\mathrm{R}=\mathrm{CH}_{2} \mathrm{CH}_{3}$;
$\mathrm{IC}_{50}=33.38 \mu \mathrm{M}$
c : $\mathrm{R}=\mathrm{CH}\left(\mathrm{CH}_{3}\right)_{2}$;
$\mathrm{IC}_{50}=6.63 \mu \mathrm{M}$
d : $\mathrm{R}=\mathrm{CH}_{2} \mathrm{CH}\left(\mathrm{CH}_{3}\right)_{2} ; \mathrm{IC}_{50}=0.185 \mu \mathrm{M}$
e : $\mathrm{R}=\mathrm{CH}_{2} \mathrm{Cl}$;
$\mathrm{IC}_{50}=0$
f : $\mathrm{R}=\mathrm{CH}_{2} \mathrm{CH}_{2} \mathrm{Br}$;
$\mathrm{IC}_{50}=5.08 \mu \mathrm{M}$
g: $\mathrm{R}=\mathrm{C}_{6} \mathrm{H}_{5}$;
$\mathrm{IC}_{50}=24.30 \mu \mathrm{M}$
$\mathbf{h}: \mathrm{R}=\mathrm{CH}_{2} \mathrm{C}_{6} \mathrm{H}_{5}$;
$\mathrm{IC}_{50}=0.045 \mu \mathrm{M}$
Selegiline;
$\mathrm{IC}_{50}=0.020 \mu \mathrm{M}$
Iproniazid;
$\mathrm{IC}_{50}=7.45 \mu \mathrm{M}$
Rasagiline;
$\mathrm{IC}_{50}=0.069 \mu \mathrm{M}$<smiles>CC(C)(C)C#N</smiles>

7-(3-chlorobenzyloxy)-4-carboxaldehydecoumarin

Fig. 67 Molecular structures of the compounds $\mathbf{C 8 4 a - C 8 4}$ 

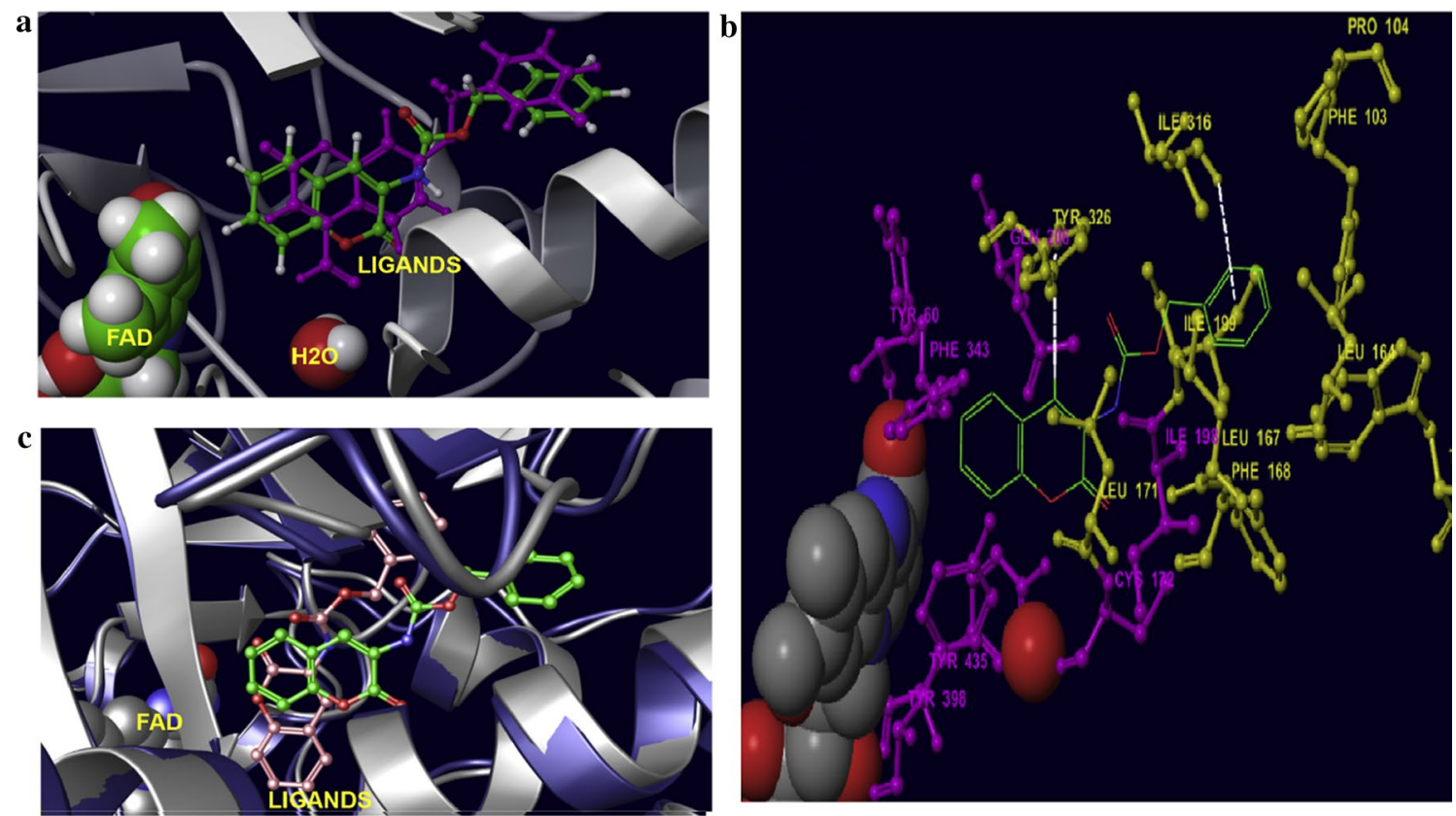

Fig. 68 Comparison of the co-crystallized ligand $\mathbf{C 8 4 i}$ (purple color) and the calculated pose for the compound C84h (colored by element, green carbons) by the docking simulation in the hMAO-B $(\mathbf{a}, \mathbf{b}, \mathbf{c})[95]$

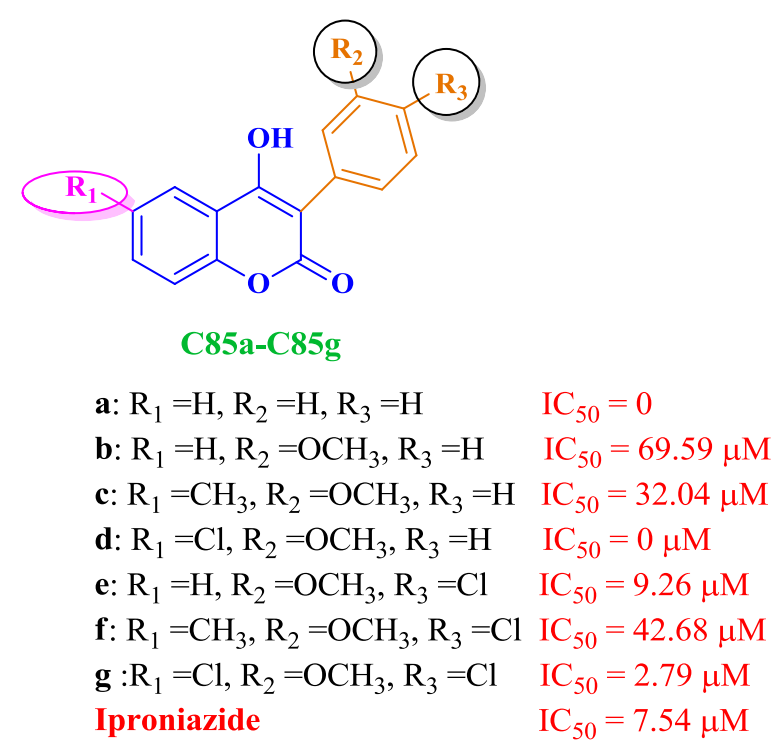

Fig. 69 Molecular structures of the compounds $\mathbf{C 8 5 a - C 8 4 g}$

ring was observed in the docking stimulations, when $\mathrm{OH}$ group was present at ortho position of the 3-phenyl ring (C68). However these conformations were sterically prohibited when methoxy/bromo group was present at the same position and abolished the activity against hMAOB (C66 and C67). Results showed that para or meta substituents in the 3-phenyl ring allowed the compound to occupy the hydrophobic subpocket making it considerably active (C63 and C73) (Fig. 64a, b). Moreover, it was also clear by comparing the predicted binding mode of compound C63 to MAO-A (Fig. 64c) and MAO-B (Fig. 64d) that, the entrance cavity only exists in MAO-B and its formation was not affected by the presence of Ile199 residue whereas, in MAO-A its formation was prevented by the presence of the Phe 208 residue in the same position as MAO-B (Fig. 64) [94].

Matos et al. [85] also synthesized another series of 6-methyl-3-phenylcoumarins $\mathbf{C 7 4 - C 7 7}$ as potent $\mathrm{MAO}-\mathrm{A}$ and MAO-B inhibitors. Results showed the compounds to be selective against isoenzyme MAO-B displaying very good $\mathrm{IC}_{50}$ values in lower nanomolar range. Derivatives $\mathbf{C 7 4}, \mathbf{C 7 5}$ and $\mathbf{C 7 7}$ showed very good inhibition and in particular compound C77, with the methoxy substituent at meta position was found to be 24 times more potent and selective than the reference compound $\mathrm{R}$-(-)-deprenyl $\left(\mathrm{IC}_{50}=19.60 \mathrm{nM}\right)$ displaying $\mathrm{IC}_{50}$ value of $0.80 \mathrm{nM}$. On the other hand, compound $\mathbf{C 7 6}$ with ortho methoxy group does not displayed any inhibitory activity even at the highest tested concentration level making it clear that the ortho position is not favorable for enzymatic inhibition (Fig.. 65) [85].

In the same context Matos et al. [96] designed two series of amino (C78-C80) and nitro 3-arylcoumarins (C81-C83) and compared them as hMAO-A and hMAO-B inhibitors [95]. The overall result showed the 

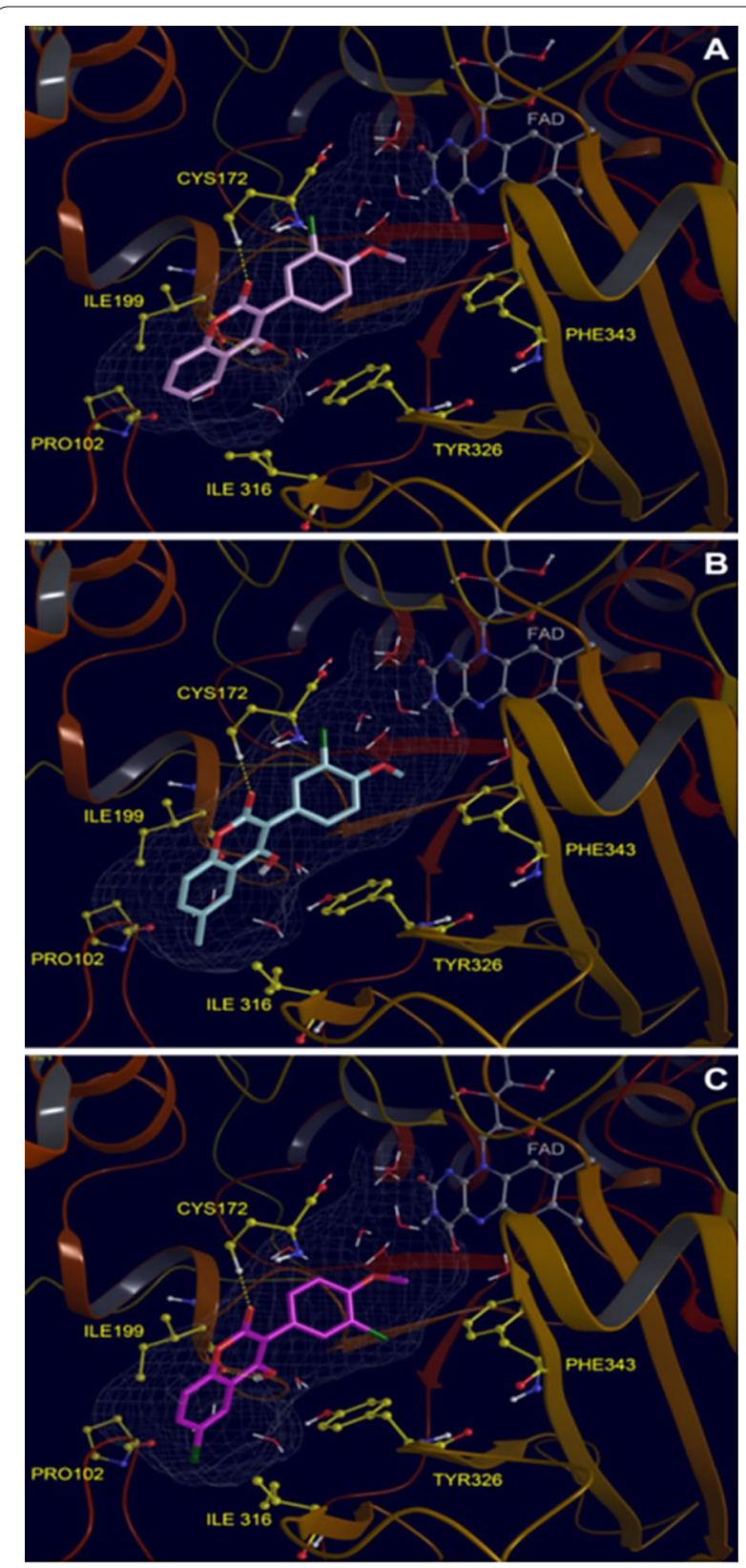

Fig. 70 Best docking poses retrieved for compounds $\mathbf{C 8 5 e}(\mathbf{A}), \mathbf{C 8 5 f}$ (B) and $\mathbf{C 8 5 g}$ (C) into the MAO-B (PDB code: 2V60). Coumarins are represented in tube with carbon atoms colored in plum for $\mathbf{C 8 5 e}$, turquoise for $\mathbf{C 8 5 f}$ and purple for $\mathbf{C 8 5 g}$ [97]

compounds to be selective against isoenzyme hMAOB. Comparative study revealed that amino compounds were more potent and selective against MAO-B than the nitro compounds, though the nitro compounds were also found to display quite good MAO-B inhibitory properties. The comparative $\mathrm{IC}_{50}$ values are displayed in Fig. 66 against the standard selegiline with $\mathrm{IC}_{50}$ value $19.60 \mathrm{nM}$.
In continuation to their work, Matos et al. [95] synthesized a series of (coumarin-3-yl)carbamates C84aC84h, and subjected them against MAO inhibitory activity (Fig. 67). Interestingly, the compounds were reported to be selective MAO-B inhibitors and their selectivity depend on their substituents linked to the carbamate group, whereas none of the synthesized derivatives exhibited activity against MAO-A isoform even at the highest concentration level of $100 \mu \mathrm{M}$ [95]. Among all the compounds, derivative C84h (benzyl(coumarin3-yl)carbamate), was found to be the most active, which displayed $\mathrm{IC}_{50}$ value of $0.045 \mu \mathrm{M}$, comparable with selegiline, the reference compound with $\mathrm{IC}_{50}$ of $0.020 \mu \mathrm{M}$. The $\mathrm{IC}_{50}$ values of all the compounds with their substituents are displayed in Fig. 67.

Further molecular docking study was performed to get the insight of the enzyme-inhibitor key interaction, which contributes, to the most stable complex conformation. It was found that the length of the compound $\mathbf{C 8 4 h}$ causes the coumarin ring's position to shift towards the FAD, compared to the co-crystallized coumarin C84i (7-(3-chlorobenzyloxy)-4-carboxaldehydecoumarin) in $2 \mathrm{~V} 60$ and the benzyl group was found positioned towards the hydrophobic pocket in the entrance cavity establishing hydrophobic interactions with residues Phe103, Pro104, Trp119, Leu164, Leu167, Phe168, Ile199 and Ile316 (Fig. 68a). Different residues were found to interact with the ligand through van der Waals interactions (Fig. 68b), mainly defined by Ile199, Leu171, Gln206, Tyr326, Ile198, Tyr398 and Phe168. In addition to these, docking results without water molecules showed a conformation for compound C84h placed deeply in the cavity (Fig. 68c).

Serra et al. [97] synthesized a series of 3-aryl-4-hydroxycoumarin derivatives (C85a-C85g) as selective $\mathrm{MAO}-\mathrm{B}$ inhibitors by introducing various groups in the 3-phenyl ring attached to the coumarin core and none of the compounds were found to show MAO-A inhibitory activity even at the highest concentration level of $100 \mu \mathrm{M}$ (Fig. 69) [97]. The MAO inhibitory activity was evaluated in vitro by the measurement of the enzymatic activity of human recombinant MAO isoforms expressed in BTI insect cells infected with baculovirus. It was reported that para methoxy $\left(\mathbf{C 8 5 e}, \mathrm{IC}_{50}=9.26 \mu \mathrm{M}\right)$ and meta chloro groups $\left(\mathbf{C 8 5 f}, \mathrm{IC}_{50}=42.68 \mu \mathrm{M}\right)$ in the 3-phenyl ring influenced the inhibitory activity up to interesting levels. Additionally, it was also reported that the presence of a chloro atom in the six position of coumarin ring in case of compound $\mathbf{C 8 5} \mathbf{g}\left(\mathrm{IC}_{50}=2.79 \mu \mathrm{M}\right)$, improved the inhibitory activity and selectivity as compared to the standard iproniazide $\left(\mathrm{IC}_{50}=7.54 \mu \mathrm{M}\right)$. Molecular docking study was performed in order to get the insight and to understand the energetically favoured orientations 


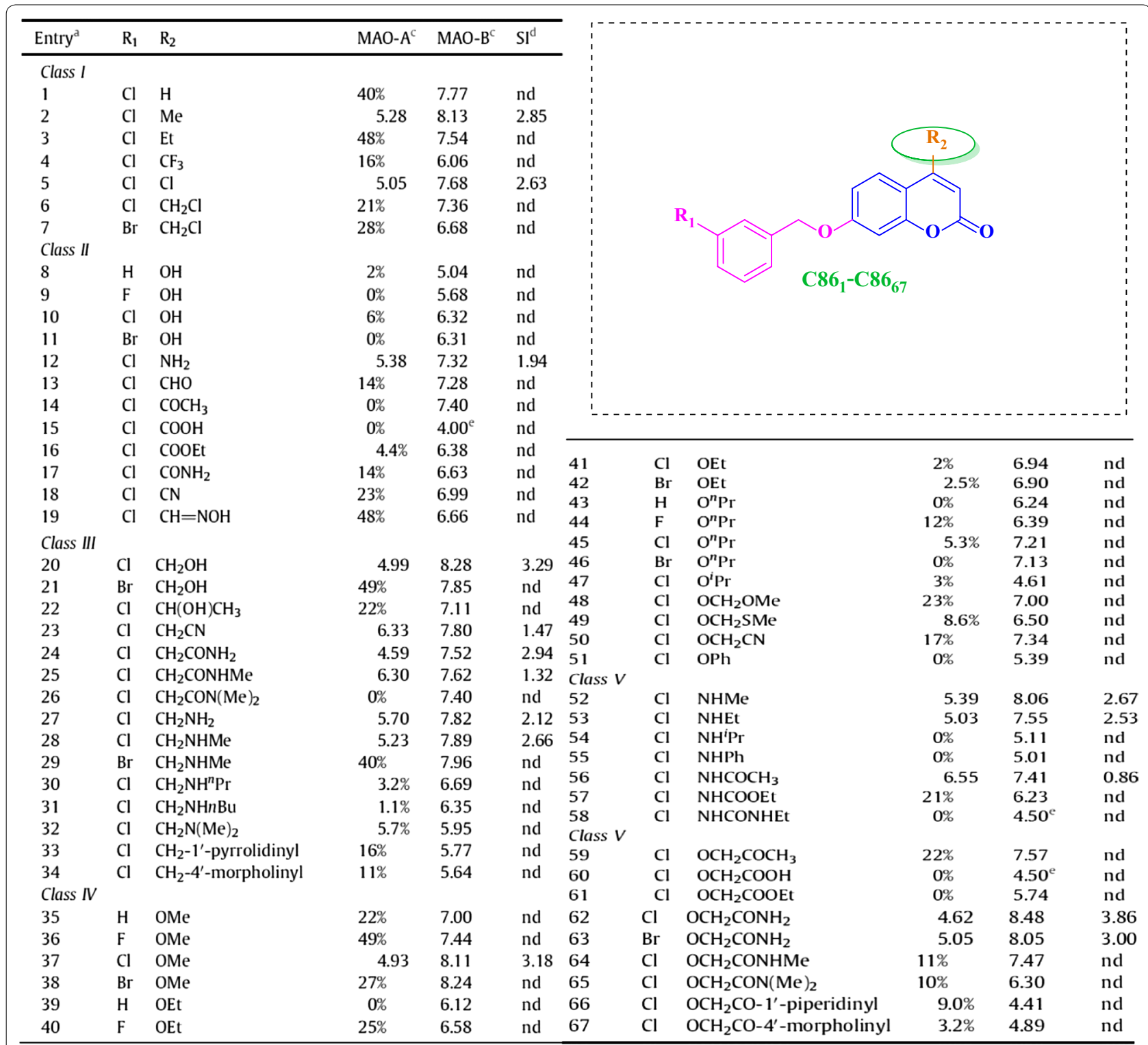

Fig. 71 Molecular structures of the compounds $\mathbf{C} 86_{1}-\mathbf{C 8 6}_{\mathbf{6 7}}$

inside the binding pocket of isoenzyme MAO-B, of the top three active compounds viz C85e, C85f and C85g. Very interestingly, same binding pattern was observed for all the three derivatives which is depicted in Fig. 70 . All the three hydroxycoumarin moieties were reported to occupy the entrance of the cavity and the 3 -arylcoumarin rings were reported to direct towards the FAD cofactor. Cys172 was involved in $\mathrm{H}$-bond formation between carbonyl oxygen of all three coumarins, hence playing its role in complex stabilization. A $\pi-\pi$ stacking interaction between Tyr326 and 3-arylcoumarin rings was also observed. Van der Waals and electrostatic interactions with Pro102, Ile316 and Phe343 were also observed for all the three hydroxycoumarin. The para- $\mathrm{OCH}_{3}$ group was reported to occupy similar position in all the three coumarins while the $\mathrm{Cl}$ substituent in the 3-phenyl ring was found to opt an opposite orientation, in case of compounds C85e and C85g, hence appearing to be unimportant for the modulation of MAO-B inhibitor activity.

In order to search potent and selective MAO-B inhibitors Pisani et al. synthesized a large library of 67 derivatives of $2 \mathrm{H}$-chromen-2-one with fine molecular tuning at position 4 of the coumarin skeleton (Fig. 71) [98]. To their interest, all the 67 compounds exhibited high MAO-B selectivity, and few were found displaying quite better potency in the low nanomolar range. For example 


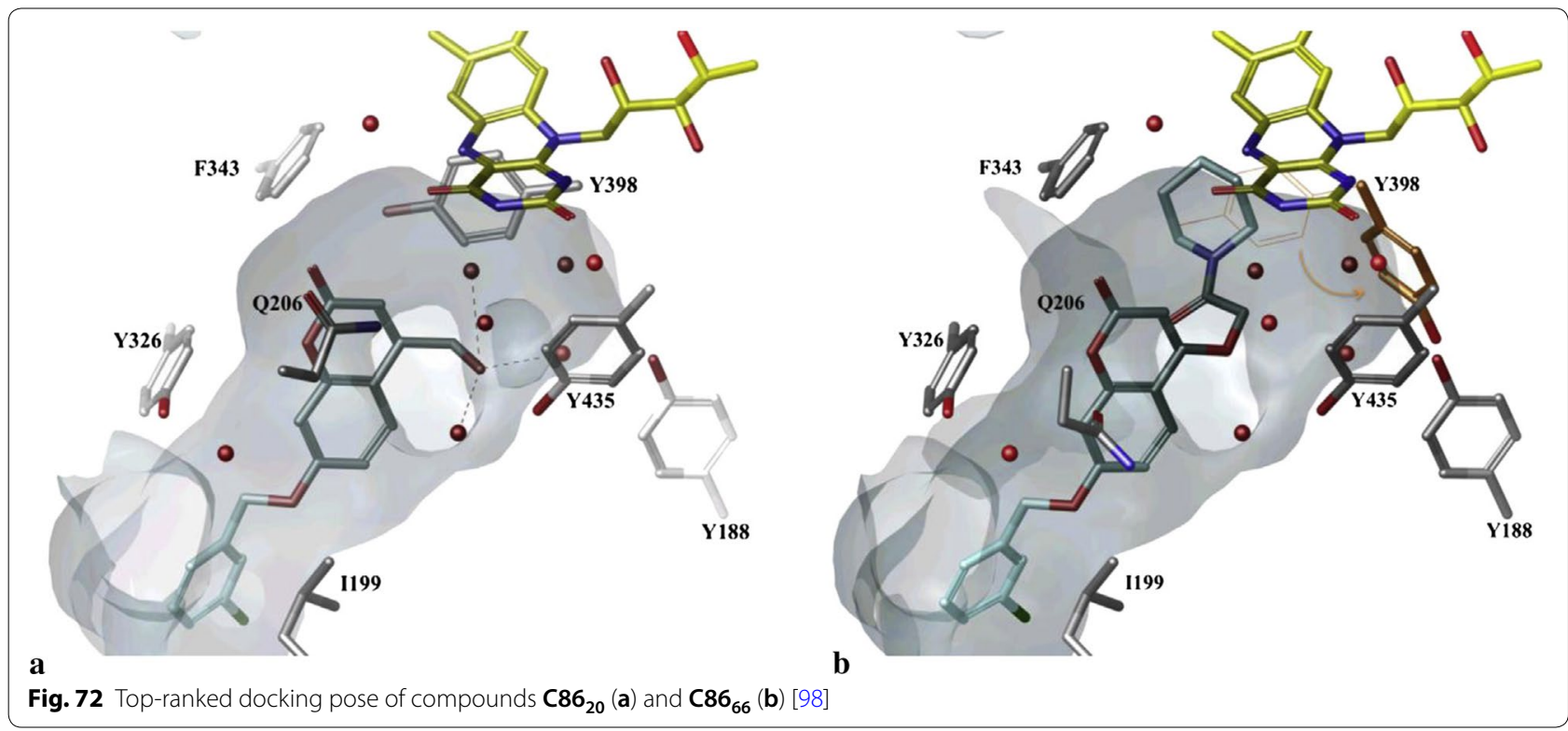

derivative, 7-metachlorobenzyloxy-4-oxyacetamido$2 \mathrm{H}$-chromen-2-one $\left(\mathbf{C 8 6}_{\mathbf{6 2}}\right)$ displayed highest MAO-B potency with $\mathrm{IC}_{50}$ value of $8.48 \mu \mathrm{M}$ and selectivity ratio of 7244, over the sub isoform MAO-A.

Molecular docking was performed to obtain the reason behind the drop of affinity and lowering the DS due to the presence of bulkier, branched or cyclic groups which cause steric crowding at position-4 of the coumarin nucleus and for this aspect, compounds C86 $_{6}$, $\mathbf{C 8 6}_{20}, \mathbf{C 8 6}_{33}, \mathbf{C 8 6}_{34}, \mathbf{C 8 6}_{51}, \mathbf{C 8 6}_{54}, \mathbf{C 8 6}_{62}$ and $\mathbf{C 8 6}_{66}$ were selected as model compounds to observe their binding interactions. To their satisfaction, they reported the experimental results in agreement with the docking results. It was found that one of the most active compound $\mathbf{C 8 6}_{20}\left(\mathrm{IC}_{50}=8.28 \mu \mathrm{M}\right)$ with 4-hydroxymethyl group was accommodated perfectly within the binding site of MOA-B, as depicted in Fig. 72a, and formed stabilized $\mathrm{HB}$ network, by involving three structural water molecules and for this case the DS was calculated be $79.85 \mathrm{~kJ} / \mathrm{mol}$. When a chlorine atom replaced the 4-hydroxymethyl group ( $\mathbf{C 8 6}_{\mathbf{6}}$ ) then DS was calculated to be $76.61 \mathrm{~kJ} / \mathrm{mol}$ and also a slight decrease in the activity was reported $\left(\mathrm{IC}_{50}=7.36 \mu \mathrm{M}\right)$, which was justified on the basis of loss of $\mathrm{HB}$ interactions. For the case of the most active compound $\mathbf{C 8 6}_{62}\left(\mathrm{IC}_{50}=8.48 \mu \mathrm{M}\right)$, possessing a large polar group at position-4 of the coumarin nucleus, displayed $\mathrm{HB}$ interactions with specific residues of amino acids and thereby increasing the the DS $(74.76 \mathrm{~kJ} / \mathrm{mol})$. Compound $\mathbf{C 8 6}_{66}$ with a bulky substituent at position-4 was found to rearrange in a high-energy folded conformation, by the displacement of Y398 from its native position to reduce possible steric clashes and hence expressing very low DS $(34.79 \mathrm{~kJ} / \mathrm{mol})$, and loss of activity $\left(\mathrm{IC}_{50}=4.41\right)$ (Fig. $\left.72 \mathrm{~b}\right)$. Similar reasons were reported for the relevant drop in DS, in case of compounds C86 $_{34}\left(\mathrm{IC}_{50}=5.64 \mu \mathrm{M}\right.$; DS $\left.56.14 \mathrm{~kJ} / \mathrm{mol}\right)$ and $\mathbf{C 8 6}_{33}$ $\left(\mathrm{IC}_{50}=5.77 \mu \mathrm{M} ; \mathrm{DS} 66.87 \mathrm{~kJ} / \mathrm{mol}\right)$ having cyclic substituents at position-4 of coumarin nucleus and hence trying for larger room and conformational rearrangements into the MAO-B binding site.

\section{Coumarin analogues as Amyloid $\beta$ peptide (AB) inhibitors}

De novo, coumarins are known for their bioactivity profile. Synthetic as well naturally occurring coumarin derivatives display a wide range of biological properties such as anti HIV [99], antitumor [100], anti-cytotoxicity [101], anti-inflammatory [102] and anti-dengue [103] to mention a few. Therefore, in order to develop the multifunctional compounds, to combat cause and symptoms of neurodegeneration, Piazzi et al. introduced halophenylalkylamidic substituents at position 6 and 7 of the coumarin moiety as dihalophenyl acid that have been repeatedly reported as BACE1 inhibitors [15]. Inhibitors of BACE1 ( $\beta$-secretase) have a great potential to be developed into anti-dementia drugs as it is believed that they help in lowering $\beta$-amyloid accumulation as reported by Jin et al. [26]. Piazzi et al. synthesized these derivatives $\left(\mathbf{C 8 7} 7_{\mathbf{G}-\mathbf{N}}\right)$ via parallel synthesis procedure, in 2 series of 4 distinct reactors (Scheme 1). The inhibitory activity of the synthesized compound was first studied against hAChE (Human Acetylcholinesterase) using Ellman's method to 
Yusufzai et al. Chemistry Central Journal

(2018) 12:128

Page 41 of 57<smiles>CCN(Cc1ccccc1)Cc1ccc(-c2cc3ccccc3oc2=O)cc1</smiles><smiles>CCN(Cc1ccccc1)Cc1ccc(-c2cc3cc(O[Hg]C)ccc3oc2=O)cc1</smiles>

ABB

b.<smiles>CCN(Cc1ccccc1)Cc1ccc(-c2cc3ccccc3oc2=O)cc1</smiles>
$c$

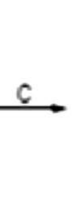<smiles>CCN(Cc1ccccc1)Cc1ccc(-c2cc3ccccc3oc2=O)cc1</smiles><smiles>[R]C(=O)NCCOc1cc2cc(-c3ccc(CN(CC)Cc4ccccc4)cc3)c(=O)oc2cc1[Al]</smiles>

Where

$\mathrm{G} ; 6 \mathrm{R}=$

$\mathrm{K} ; 7 \mathrm{R}=$<smiles>Cc1cc(F)cc(F)c1</smiles>

$\mathrm{H} ; 6 \mathrm{R}=$

$\mathrm{L} ; 7 \mathrm{R}=$<smiles>C=Cc1cc(F)cc(F)c1</smiles>

$\mathrm{I} ; 6 \mathrm{R}=$<smiles>[R]#[W]C#[W]</smiles>

$\mathrm{J} ; 6 \mathrm{R}=$<smiles>C=Cc1ccc(Cl)c(Cl)c1</smiles><smiles>C=Cc1ccc(Cl)c(Cl)c1</smiles>

Scheme 1 Synthesis of the studied coumarin compounds ( $\mathbf{C 8 7}_{\mathbf{G - N}}$ ). Reagents: (a) $\mathrm{HBr}$; (b) 2-(Boc-amino)ethyl bromide; (c) $\mathrm{CF}_{3} \mathrm{COOH}_{\text {; }}(\mathrm{d}) \mathrm{DCC}_{\text {, }}$ (3,5-difluorophenyl)acetic acid, 3,5-difluorocinnamic acid, 3,4-difluorodihydrocinnamic acid or 3,4-dichlorocinnamic acid [15]

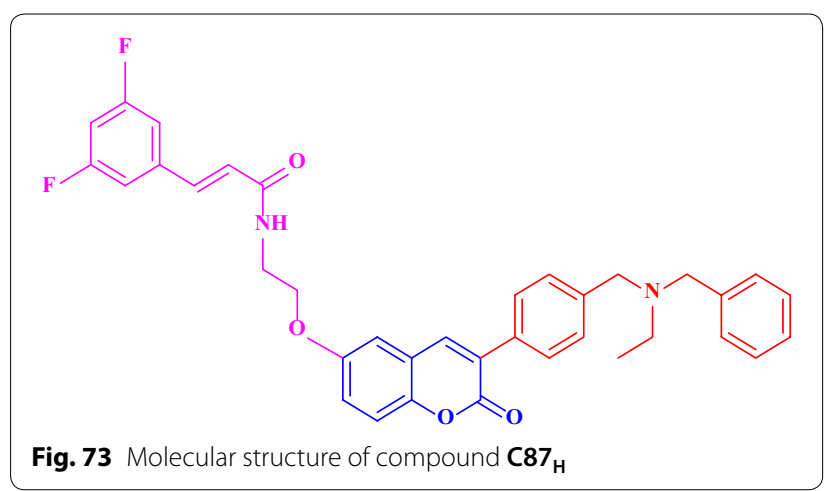

determine the rate of hydrolysis of acetylcholine and secondly against BACE1, using spectrofluorometric method. Out of all the derivatives synthesized, compound $N$ - $(2-$ ((3-(4-((benzyl(ethyl)amino)methyl)phenyl)-2-oxo-2Hchromen-6-yl)oxy)ethyl)-3-(3,5-difluorophenyl) acryl amide $\left(\mathbf{C 8 7}_{\mathbf{H}}\right)$ showed the most potential as BACE1 inhibitor with $\mathrm{IC}_{50}$ value $0.099 \mu \mathrm{M}$ (Fig. 73).

Compound $\mathbf{C 8 7}_{\mathbf{H}}$, was then further docked to the active site of BACE1. The result obtained showed that the compound gave heterogeneous docking poses due to the flexibility of the substituent at position 6 of the coumarin moiety. $N$-Benzyl group were found to interact with 


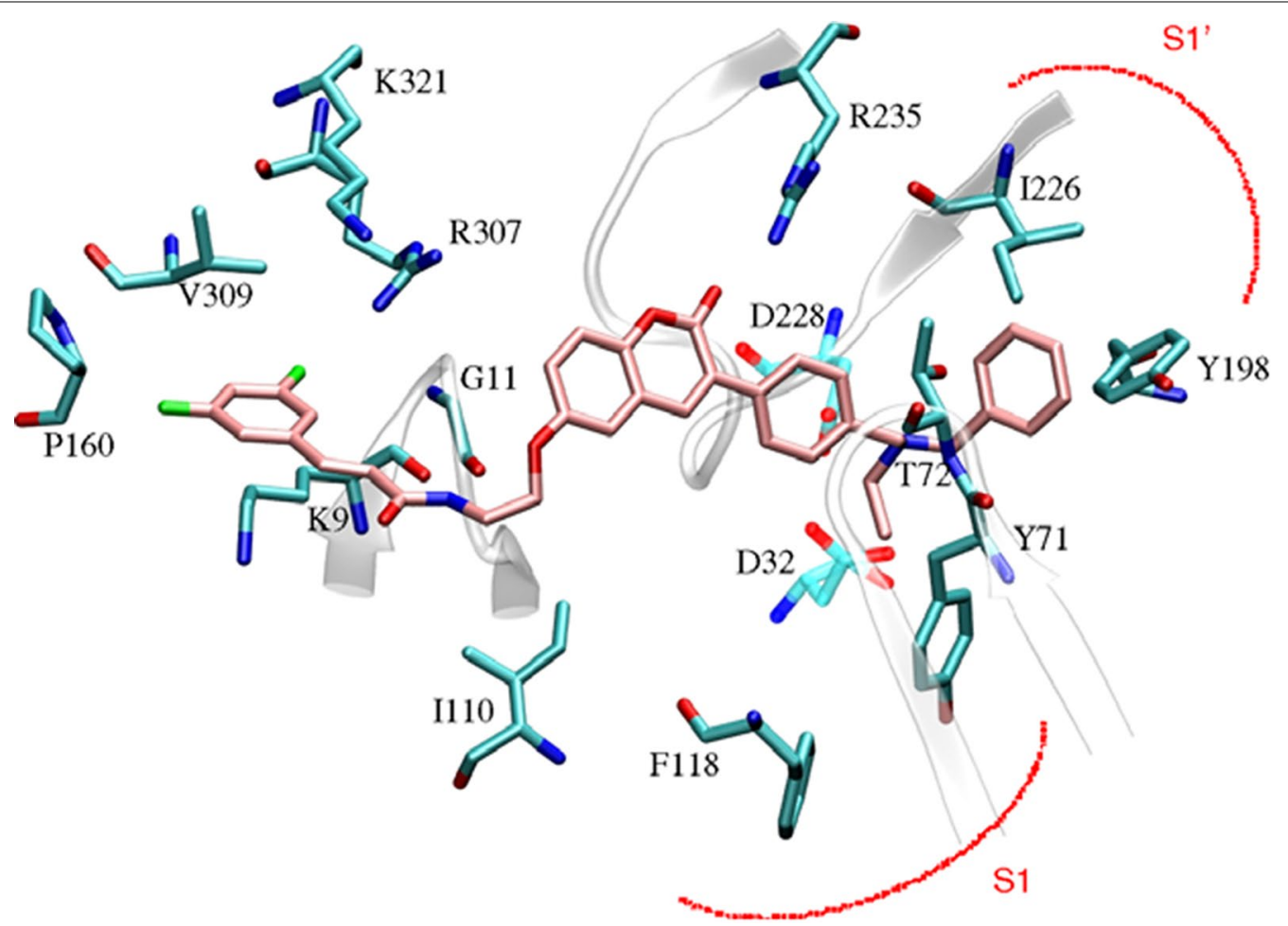

Fig. 74 Proposed binding mode of compound $\mathbf{C 8 7}_{\mathbf{H}}$ docked at the active site of BACE1 [15]<smiles>CC(=O)c1cc2ccccc2oc1=O</smiles>

Fig. 75 Molecular structure of compound $\mathbf{C 8 8 a}$ and $\mathbf{C 8 8 b}$

Tyr198 and Ile226, whereas the $\mathrm{N}$-ethyl portion lied on the aromatic ring of Tyr71. The coumarin core that has 3-phenyl substituent was embedded into the S2 pocket of the enzyme with the carbonyl group being able to form hydrogen bond with the side chain of Arg235 (Fig. 74). Piazzi et al. [15] stated that the flexibility of the bridging amino ethoxy segment and relative structural permittivity of the enzyme portion towards the presence of a substituent at position 6 or 7 of the coumarin nucleus contributed to the relative binding affinity of this series of molecules. The orientation of the substituents at position 6 might be stabilized by the interaction of the amidic nitrogen with Gly11 whereas the 3,5-difluorophenyl portion may possibly be accommodated into a peripheral cationic spot formed by residues Lys9, Arg307, Lys321, Pro160 and Val309 [15].
Recently, Ranade et al. [104] studied coumarin thiosemicarbazone (C88b) as inhibitors of amyloid $\beta$ peptide $(A \beta)$ aggregation, as accumulation of $A \beta$ peptide plays an important role in the progression of AD. They used different methods to study the inhibition of $A \beta$ peptide for instance, ThT fluorescence assay, turbidity assay, 1-anilinonaphthalene-8-sulfonic acid (ANS) fluorescence assay and native gel electrophoresis. Interestingly the results from ThT fluorescence assay gave the insight that coumarin thiosemicarbazone has more ability to inhibit aggregation of $A \beta$ peptide as compared to 3 -acetyl coumarin (C88a) while the turbidity assay gave the information about increase in aggregation of $A \beta$ peptide during initial stages and significant decrease in aggregation lately, for both the coumarin types. Therefore, from both these assays it was concluded that coumarin thiosemicarbazone inhibits the aggregation of $\mathrm{A} \beta$ peptide. Assay performed by using the dye 1-anilinonaphthalene-8-sulfonic acid showed that coumarin thiosemicarbazone interacts with hydrophobic regions of $A \beta$ peptide and reduces its aggregation as compared to acetyl coumarin. Results obtained from the native gel assay revealed the ability of coumarin thiosemicarbazone to diminish the formation of high molecular weight aggregates. Hence, the overall result obtained was in favour of coumarin thiosemicarbazone rather 
<smiles>[R]c1cccc2cc(C(=O)Nc3cc(Br)ccc3N3CCN([X])CC3)c(=N)oc12</smiles>

C89a-C89c

a; $\mathrm{X}=$ Benzyl; $\mathrm{R}=\mathrm{H}$

b; $\mathrm{X}=\mathrm{Boc} ; \mathrm{R}=\mathrm{H}$

c; $\mathrm{X}=$ Benzyl; $\mathrm{R}=\mathrm{OCH}_{3}$<smiles>[X]c1ccc2c(c1)C(=O)N(CCC)C2=O</smiles><smiles>[Y][Si]</smiles>

C90a-C90c

a; $\mathrm{X}=$ Benzyl; $\mathrm{R}=\mathrm{H}$

b; $\mathrm{X}=\mathrm{Boc} ; \mathrm{R}=\mathrm{H}$

c; $\mathrm{X}=$ Benzyl; $\mathrm{R}=\mathrm{OCH}_{3}$<smiles>[X]N1CCN(c2ccc(Br)cc2NC(=O)c2cc3ccccc3o/c2=N\c2ccccc2)CC1</smiles>

C91a-C91m

a; $\mathrm{X}=\sim \sim \mathrm{CH}_{2} \mathrm{CH}_{2} \mathrm{OH}$

b; $\mathrm{X}=\sim \sim \mathrm{CH} 2 \mathrm{CH}_{2} \mathrm{CN}$

c; $\mathrm{X}=$

$\mathrm{d} ; \mathrm{X}=$<smiles>CCc1ccc(OC)cc1</smiles><smiles></smiles>

Fig. 76 Molecular structure of compounds C89a-c and C90a-c and C91a-m

than acetyl coumarin and it was concluded from the study that thiosemicarbazone derivatives of 3-acetyl coumarins could play immense role as promising drug molecules for the treatment of AD (Fig. 75) [104].

Edraki et al. [105] synthesized a series of phenylimino-2H-chromen-3-carboxamide derivatives C89aC89c. C90a-C90c and C91a-C91m, as BACE1 inhibitors, which plays an active role in dissolution of aggregated $\beta$-amyloid, a peptide that is potentially responsible for enhancing the symptoms of $\mathrm{AD}$ (Fig. 76). They evaluated these derivatives for BACE1 inhibitory activity using a FRET-based assay. Furthermore docking was performed to get the insight of their binding behaviour. The results revealed that the phenyl-imino group of the scaffold establishes favourable $\pi-\pi$ stacking interaction with side chain of Phe108 of flap pocket as for the case $\mathbf{C 8 9 b}$ and $\mathbf{C 9 0 b}$. Compound C89b was close to inactive against BACE1 enzyme $\left(\mathrm{IC}_{50}>10 \mu \mathrm{M}\right)$, but on replacing the hydrogen of imino moiety of the imino-2H-chromene ring, with the phenyl moiety, increased the inhibitory potential of compound C90b $\left(\mathrm{IC}_{50}=9.61 \mu \mathrm{M}\right)$ (Fig. 77). The derivatives in which heteroatomic groups were attached to N4-piperazine moiety via an aliphatic linkage were reported to possess higher BACE1 inhibitory properties, which was due to the interactions with the residues of $S 1-S^{\prime} 1$ sub-pocket. Some other derivatives displayed high inhibitory potential at 5 and $10 \mu \mathrm{M}$ on the production of $A \beta$ in N2a-APPswe cells. The highest active compound C91e, was reported to possess $\mathrm{IC}_{50}$ value $98 \mathrm{nM}$. This significant inhibitory potential was justified on the basis of the additional hydrophobic interaction 


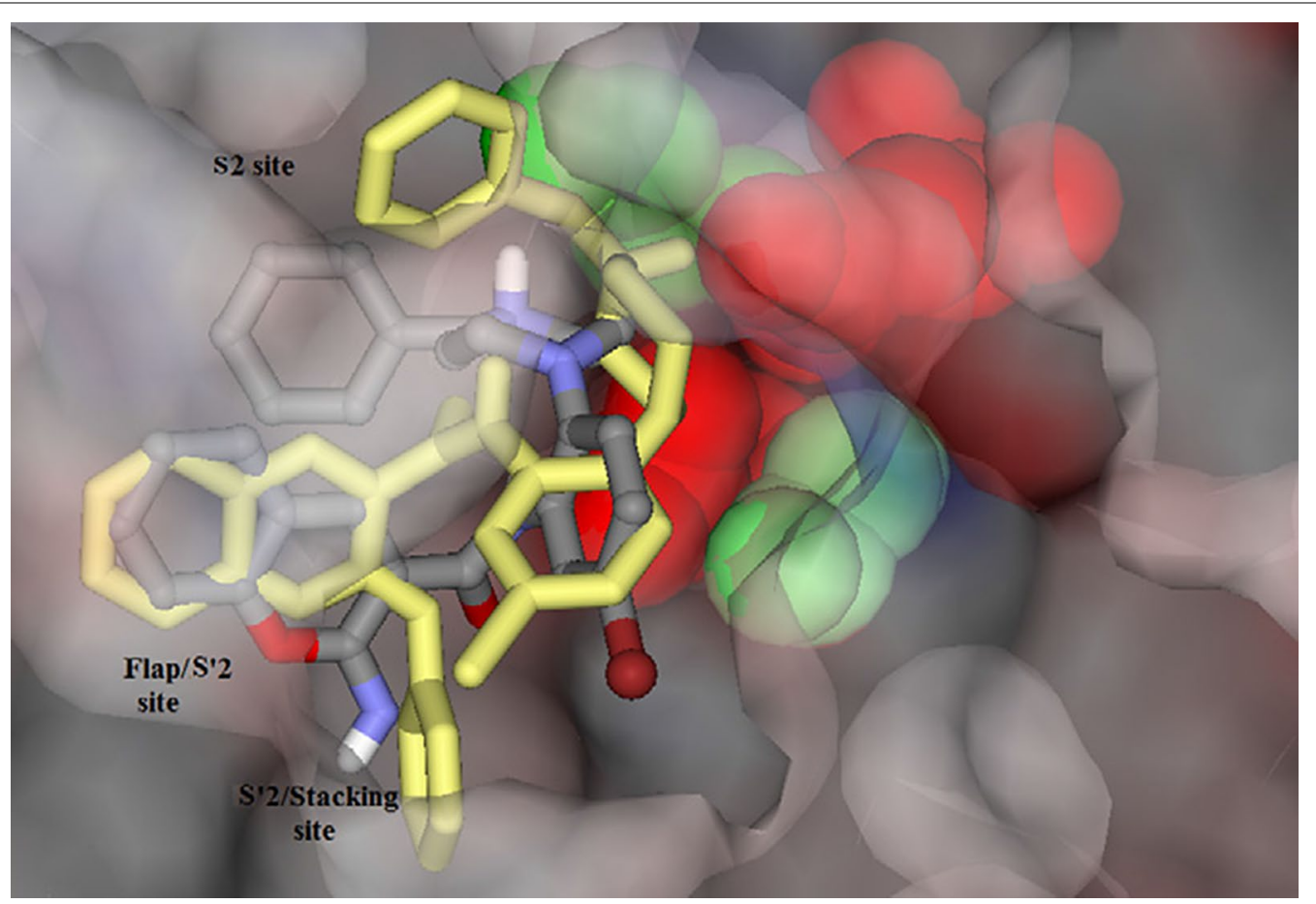

Fig. 77 The comparative binding affinities of benzylpiperazine derivative imino-2H-chromene-3-carboxamide $\mathbf{C 8 9 a}$ and its Phenylimino-2H-chro men-3-carboxamide analogue C90a [105]

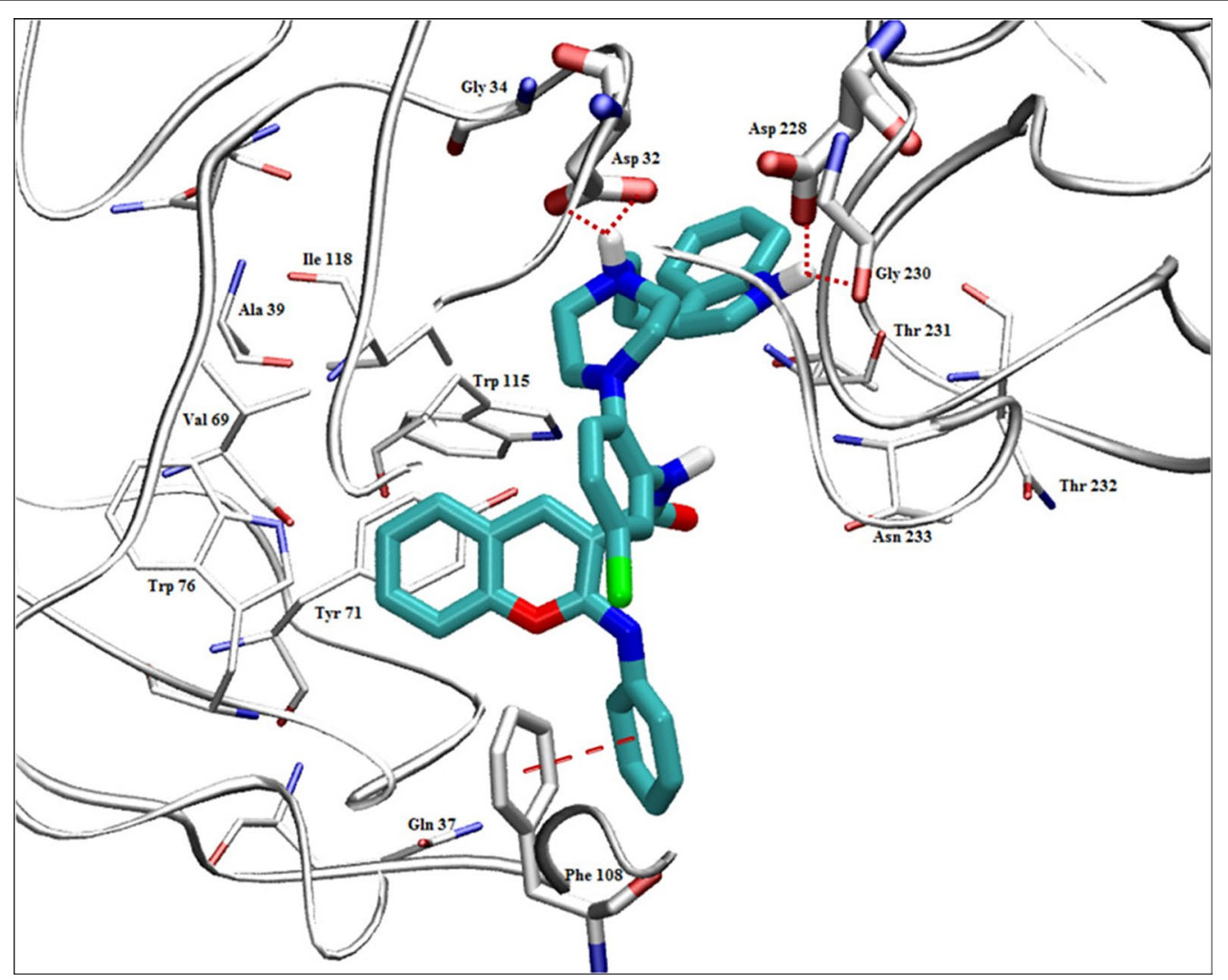

Fig. 78 Binding mode of C91h into active site of BACE1 [105] 
<smiles>Cc1cc(=O)oc2cc(OCCN3CCN(CC(=O)Nc4c5c(nc6ccccc46)CCCC5)CC3)ccc12</smiles>

Fig. 79 Molecular structure of compound C92 with the hydrophobic residue of the S2 sub-pocket of the active site and the network of hydrogen bonding interactions between the heteroatoms of phthalimide moiety and corresponding residue of the active site. On the elongation of methylene linker between phthalimide group and piperazine ring in case of compounds C91f, $\left(\mathrm{IC}_{50}=0.326 \mu \mathrm{M}\right)$ and $\mathrm{C91g}\left(\mathrm{IC}_{50}=0.854 \mu \mathrm{M}\right)$, the inhibitory properties were reported to decrease

$\mathbf{a}$

b

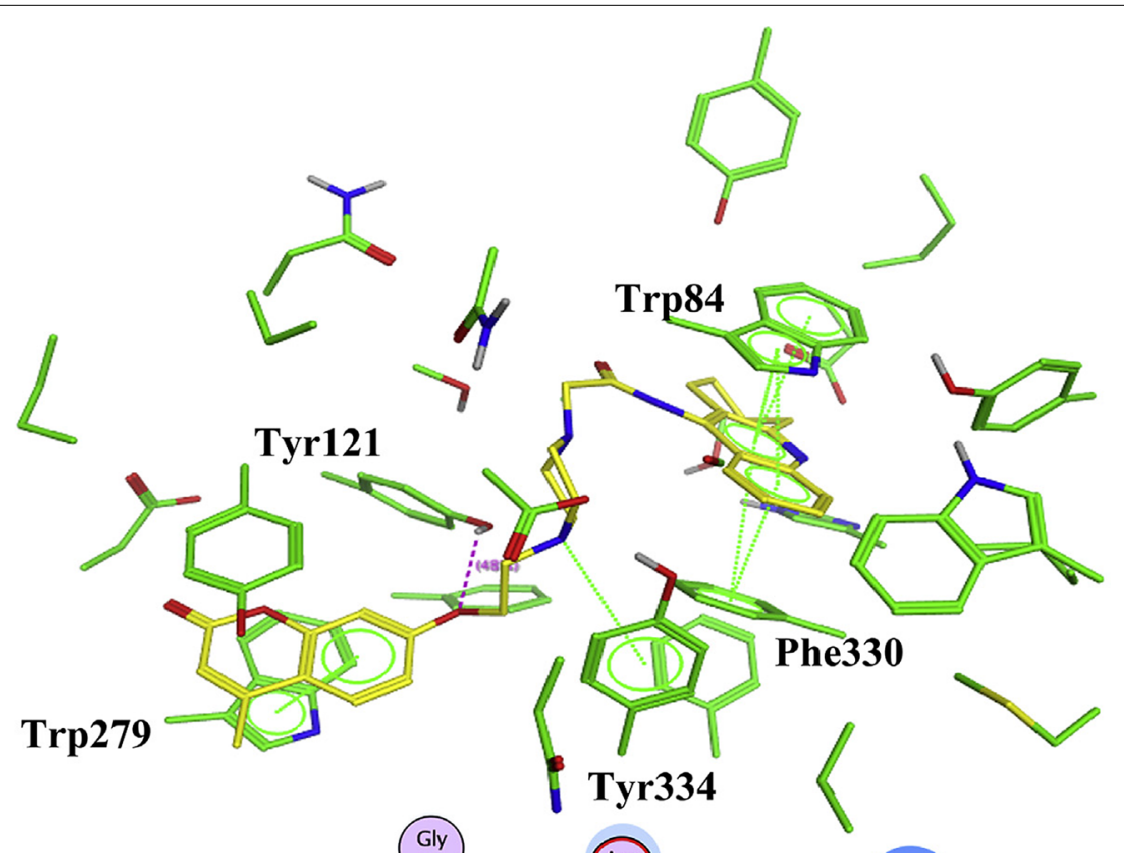

Gly
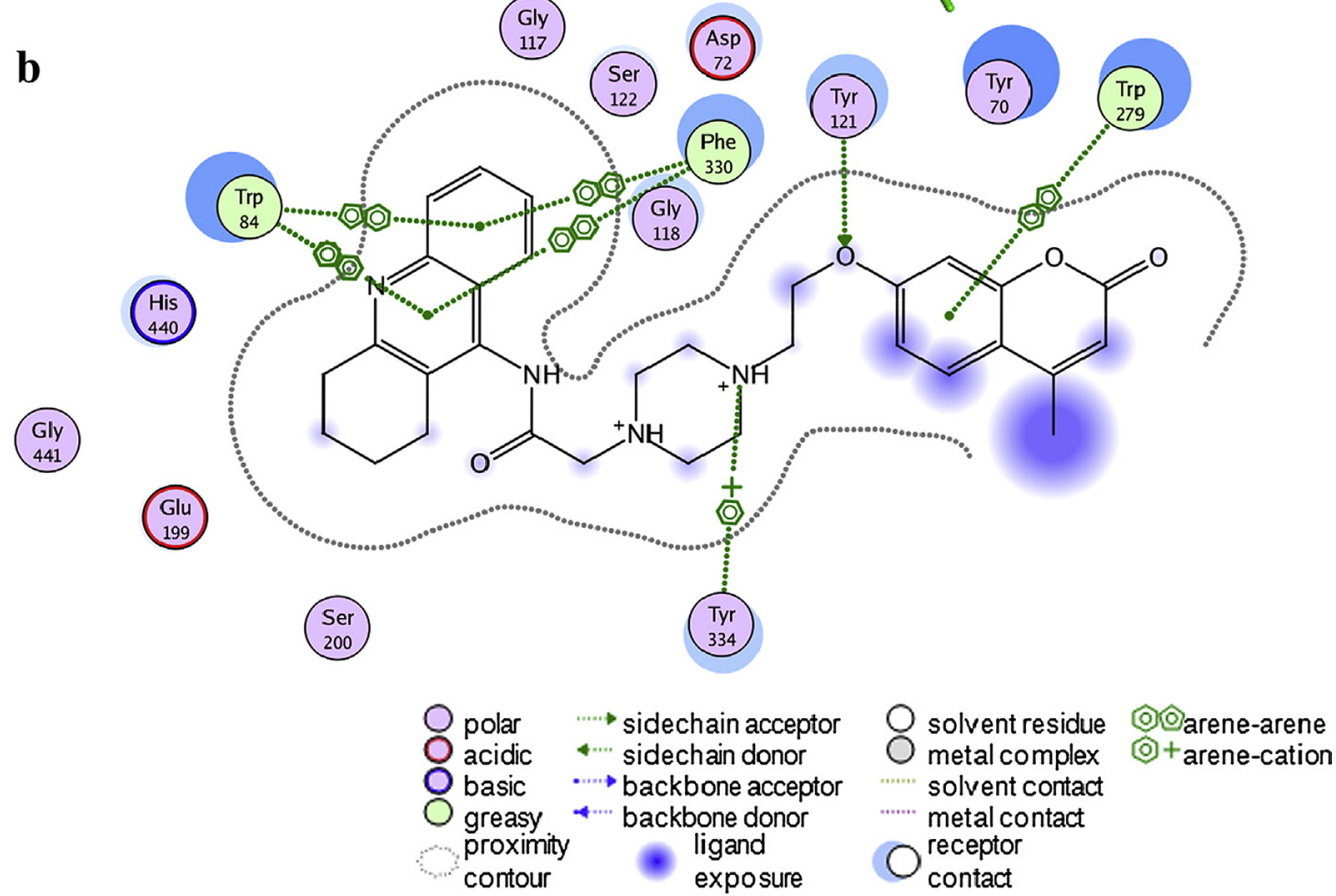

Fig. 80 3D docking model of $\mathbf{C 9 2}$ with $\operatorname{TcAChE}(\mathbf{a}, \mathbf{b})[9]$ 
<smiles>O=c1oc2cc(/N=C/c3ccccc3O)ccc2c2c1CCC2</smiles>

Fig. 81 Molecular structure of compound $\mathbf{C 9 3}$

due to hindrance of hydrogen bonding interactions at S2 sub-pocket of the active site. Moreover, it was found that introducing $1 \mathrm{H}$-indol ring into N4-piperazine of the scaffold via an ethyl linker increases the inhibition of BACE1 as observed for compound $\mathbf{C 9 1 h}$ $\left(\mathrm{IC}_{50}=0.928 \mu \mathrm{M}\right)$ (Fig. 78). More comparisons were made to understand the inhibitory behaviour of other compounds. For instance, comparison of compounds C89a and C90a demonstrated that the introduction of phenyl moiety into the imino group of benzyl piperazine derivative of imino- $2 \mathrm{H}$-chromene-3-carboxamide scaffold C89a $\left(\mathrm{IC}_{50}=1.216 \mu \mathrm{M}\right)$, resulted in enhanced inhibitory potential of compound in phenylimino-2H-chromen-3-carboxamide derivative C90a $\left(\mathrm{IC}_{50}=0.503 \mu \mathrm{M}\right)[105]$.

\section{Coumarin analogues as multifunctional inhibitors}

Xie et al. [9] designed a series of novel tacrine-coumarin hybrids as multifunctional ChE inhibitors for AD treatment. They connected tacrine to coumarin scaffold through a suitable linker. Tacrine are known as the first $\mathrm{ChE}$ inhibitor for AD treatment, before FDA withdrew it, due to its role in severe liver toxicity. Despite its withdrawal, tacrine is still a widely used scaffold in the designing of multifunctional molecules for $\mathrm{AD}$ treatment, due to its ability to inhibit ChEs through CAS. Among the tacrine-coumarin hybrids synthesized, compound 2-(4-(2-((4-methyl-2-oxo-2H-chromen-7yl)oxy)ethyl) piperazin-1-yl)-N-(1,2,3,4-tetrahydroacri-din-9-yl) acetamide (C92) showed the highest inhibitory activity against AChE (Fig. 79) [9].

Among the factors that contributed to the good AChE inhibitory activity of compound C92 was the linker length between coumarin moiety and piperazine, and the substituent at position 4 of the coumarin moiety. C92 with only two-carbon linker provided a good AChE inhibitory activity compared to other hybrids with linker length more than two carbons. The compounds, which possessed methyl substituent at position 4 of coumarin moiety, showed better AChE inhibitory activity compared to the compounds, which has $\mathrm{H}$, methoxy and phenyl group as substituent. The molecular modelling results for C92 showed, that the tacrine moiety of C92 was bound to the CAS of AChE and stacked against the phenyl ring of Phe330 and the indole ring of Trp84, whereas the coumarin ring interacted with the indole ring of Trp279 of PAS via $\pi-\pi$ stacking interactions (Fig. 80). As expected to be, the piperazine was found to bind to the middle gorge site of AChE through a cation- $\pi$ interaction, between its protonated nitrogen atom and Tyr334, enhanced by a hydrogen bond, that was formed between the oxygen atom of $\mathbf{C 9 2}$ side chain and Tyr121. Besides a favourable AChE inhibitory result, compound C92 also showed promising metal chelating property and low cell toxicity. The multifunctional ability of $\mathbf{C 9 2}$ makes it to be considered as a promising lead compound for further research and its derivatization in the future.
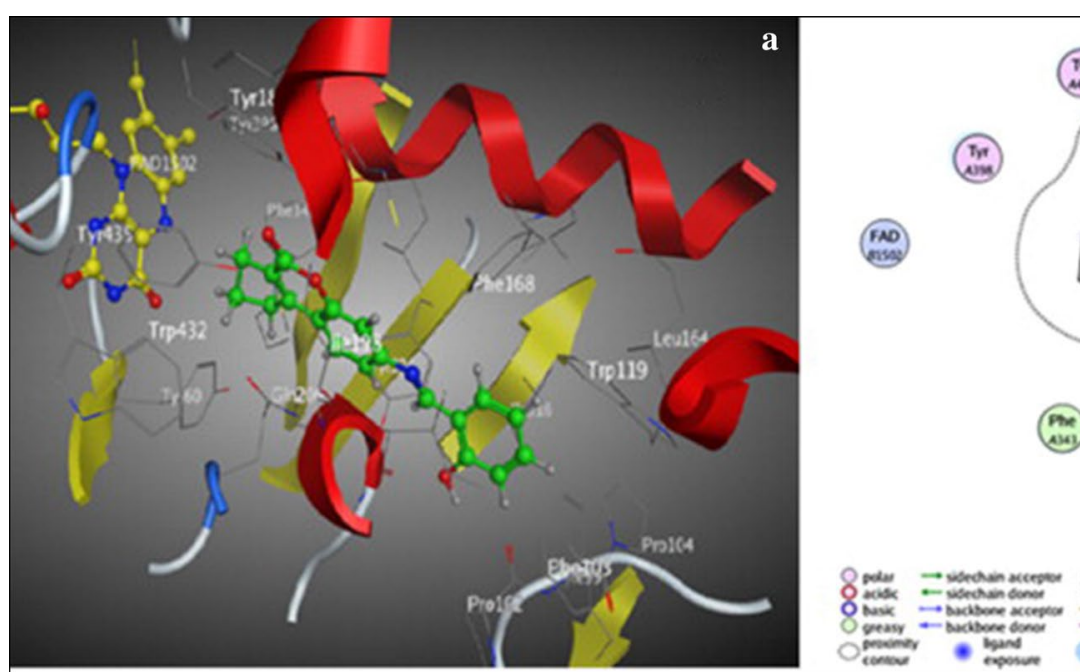

Fig. 82 Molecular docking of C93 with MAO-B (a, b) [12] 


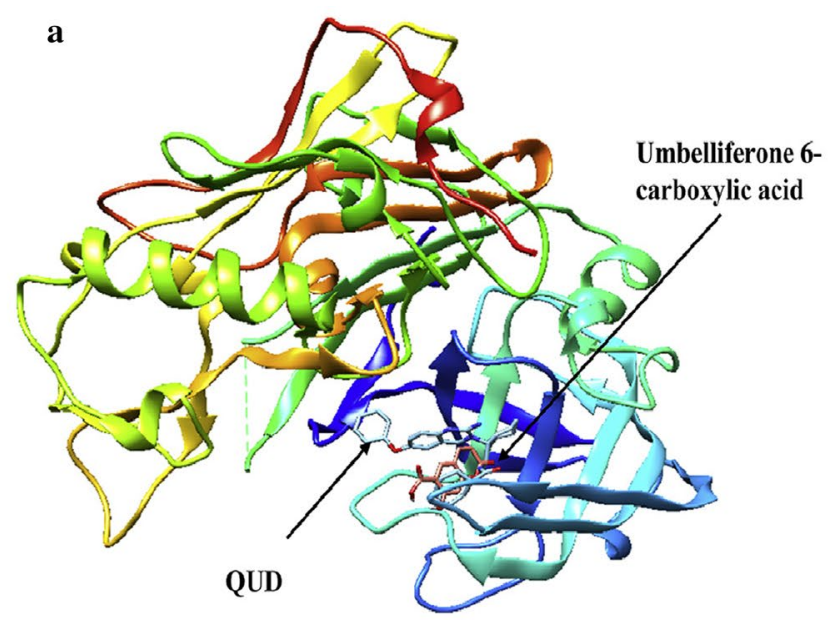

b

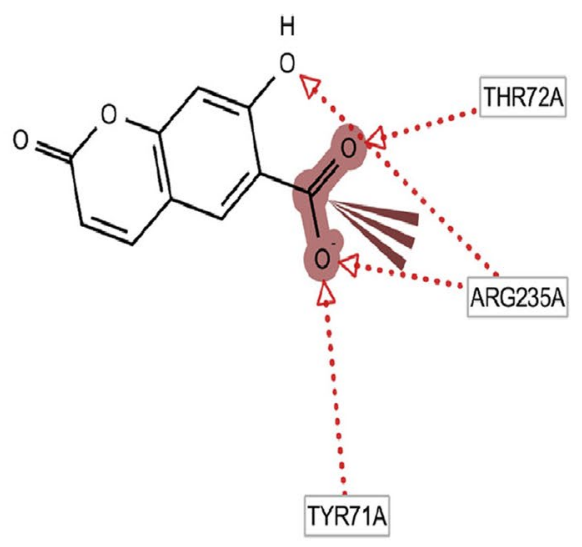

a

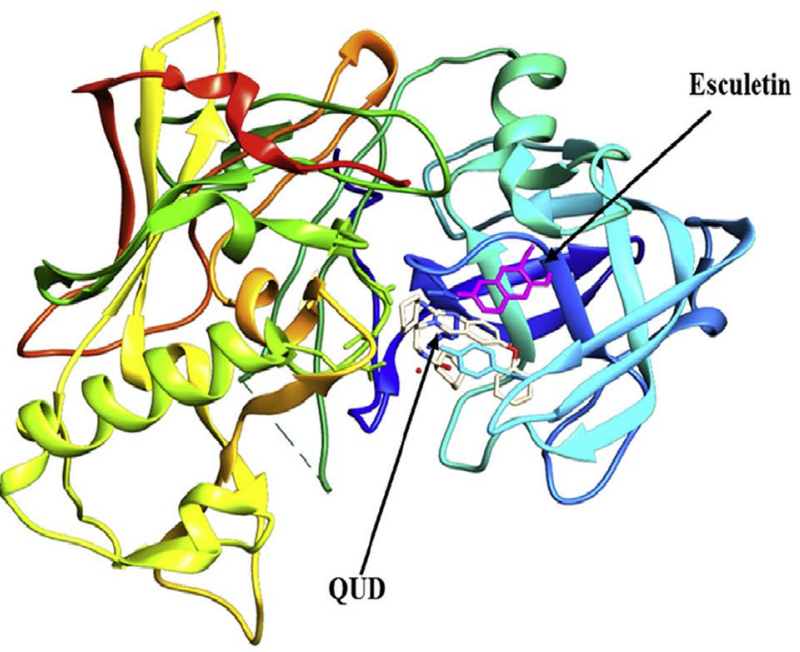

b
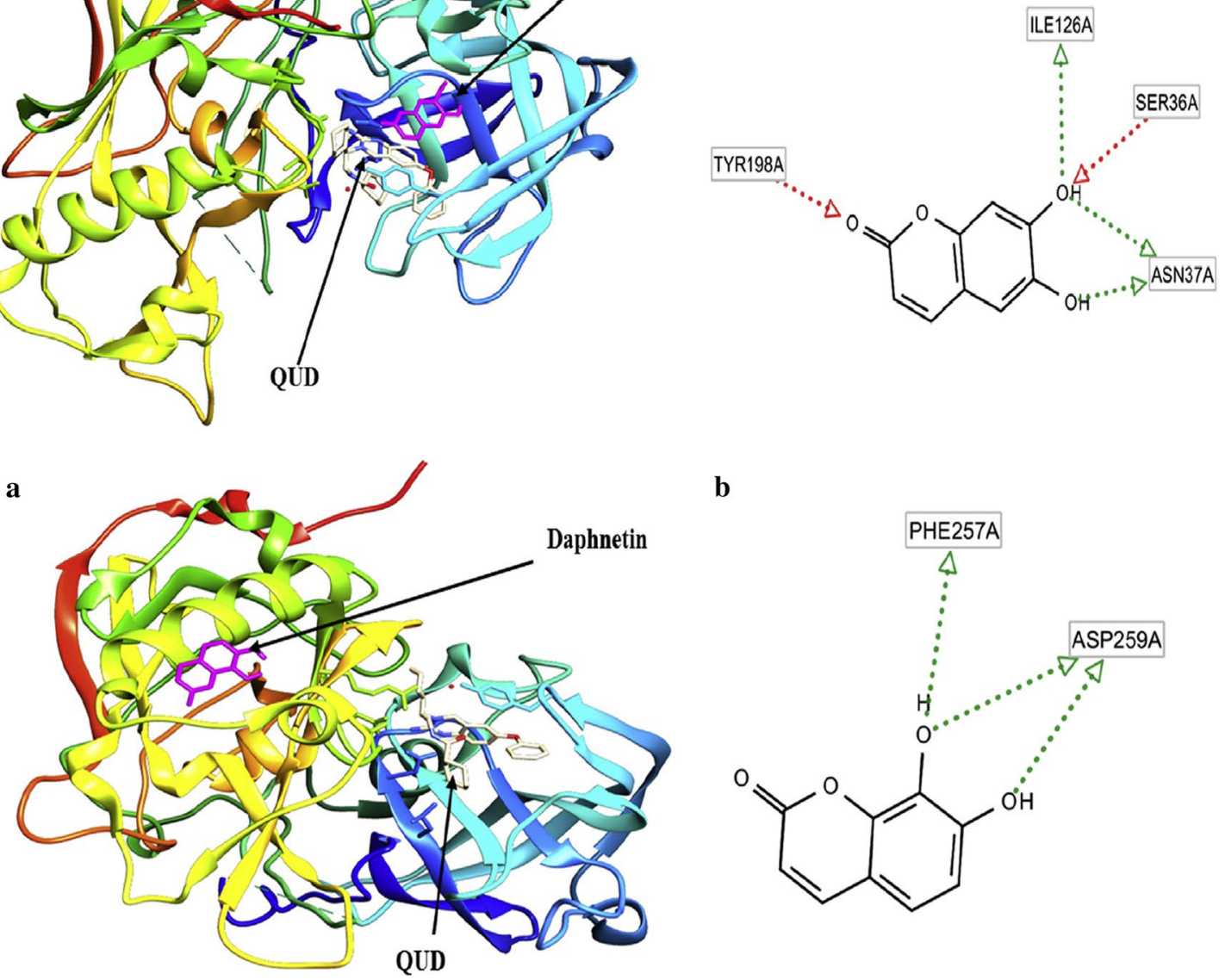

b

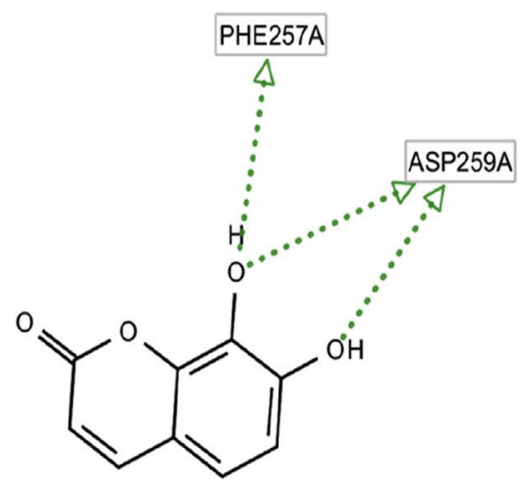

Fig. 83 a Molecular docking model BACE1 with C94b, C94h and C94j: (b) Diagram of the ligand interaction of C94b, C94 $\mathbf{h}$ and $\mathbf{C 9 4 j}$ in the active site of BACE1 [106] 


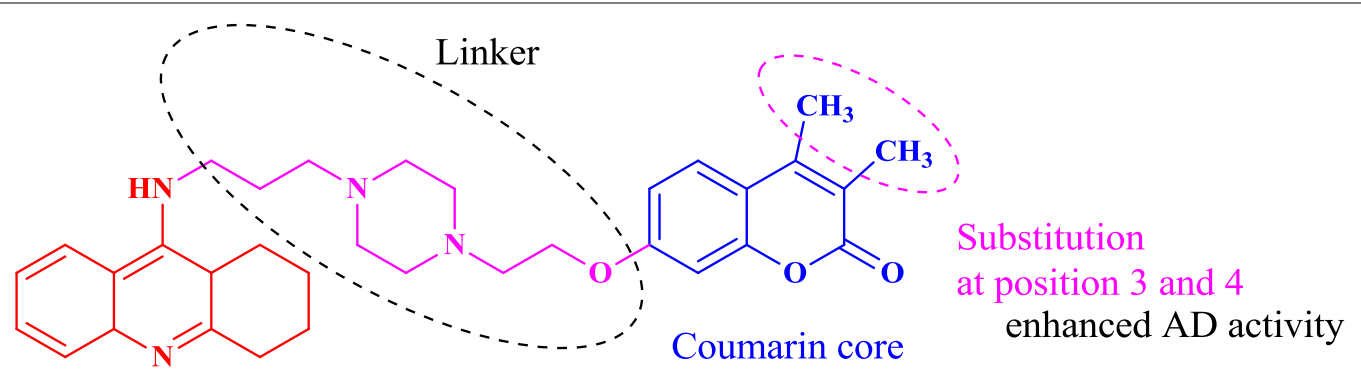

Tacrine unit

A balanced multifunctional hybrid

$$
\begin{aligned}
& \mathrm{AChE} \mathrm{IC}_{50}=33.63 \mathrm{nM} \text { for eeAChE and } 16.11 \mathrm{nM} \text { for } \mathrm{hAChE} \\
& \mathrm{BChE} \mathrm{IC}_{50}=80.72 \mathrm{nM} \text { for eqBuChE and } 112.72 \mathrm{nM} \text { for hBuChE} \\
& \mathrm{hMAO}-\mathrm{B} \mathrm{IC} \mathrm{IC}_{50}=0.24 \mu \mathrm{M}
\end{aligned}
$$

Fig. 84 Molecular structure of compound C95

Huang et al. [12] designed new multifunctional molecules endowed with the hope of developing novel multifunctional drug that are able to improve clinical symptoms as well as diminish the progression of diseases. They combined coumarin and the pharmacophore moiety of clioquinol (CQ), a renowned metal chelator. Various literatures have reported coumarin analogues as potent $\mathrm{MAO}-\mathrm{B}$ inhibitors and $\mathrm{A} \beta$ anti-aggregation, whereas CQ has been reported to help in decreasing the rate of cognitive decline in moderately severe AD patients in a phase II clinical trial. This integration was made to obtain a novel series of coumarin derivatives that were expected to be biometal chelators, as well as inhibitors of MAO- $\mathrm{B}$ and $\mathrm{A} \beta$ aggregation. The inhibitory activities of the synthesized derivatives against hMAO$\mathrm{B}$ was identified by measuring their effects on the production of $\mathrm{H}_{2} \mathrm{O}_{2}$ from p-tyramine with pargyline and iproniazid as references. Among the derivatives tested, compound 7-((2-hydroxybenzylidene)amino)-1,3,3a,9btetrahydrocyclopenta[c] chromen-4(2H)-one (C93) gave the most promising result as selective hMAO-B inhibitor when compared to the references pargyline and iproniazid (Fig. 81) [12].

Huang et al. [12] therefore stated, that the steric effect at positions 3 and 4 might have been benefited to hMAO-B inhibitory activity. The result showed by compound C93 which has cyclopentenyl substituent at the 3 and 4 positions of compound, strongly supported their hypothesis. The carbon nitrogen double bond might also have been played a part in improving its MAO-B inhibitory activity. There was a significant decrease in hMAO$B$ inhibitory activities, when the carbon nitrogen double bond was hydrogenated. Huang et al. justified this by stating that the carbon nitrogen double bond has influence on the hMAO-B inhibitory activity to different degrees. When tested for its metal-chelating effect, the result showed that there was an interaction between $\mathrm{Cu}^{2+}$ and compound $\mathbf{C 9 3}$ due to the increase of $\mathrm{Cu}^{2+}$ concentration. Based on this observation, Huang et al. concluded that the compound might have effectively chelated $\mathrm{Cu}^{2+}$ and have served as metal chelators in AD treatment. Docking studies were performed on compound C93, to identify its interaction mode with MAOB. The result obtained showed that the coumarin moiety was located within the substrate cavity of the enzyme and a hydrogen bond was observed with Tyr435 (Fig. 82). The substituent of compound $\mathbf{C 9 3}$ at position 7, was directed towards the hydrophobic pocket in the entrance cavity, establishing hydrophobic interactions with residues Phe103, Pro104, Trp119, Leu164, Leu167, Phe168, Ile199 and Ile316. Huang et al. stated that the interaction made by this compound with the enzyme is the best explanation for its good hMAO-B inhibitory activity.

It is of great importance that natural as well as synthetic coumarins, both display large range of pharmacological properties and hence they own a special place in the field of pharmaceutical research. In this respect Ali et al. isolated umbelliferone (C94a), umbelliferone 6-carboxylic acid (C94b), scopoletin (C94c), isoscopoletin (C94d), 7-methoxy coumarin (C94e), scoparone (C94f), scopolin (C94g), esculetin (C94h) and 2'-isopropyl psoralene (C94i) from Angelica decursiva and evaluated their inhibitory effects against acetylcholinesterase (AChE), butyrylcholinesterase $(\mathrm{BuChE})$, and $\beta$-site amyloid 


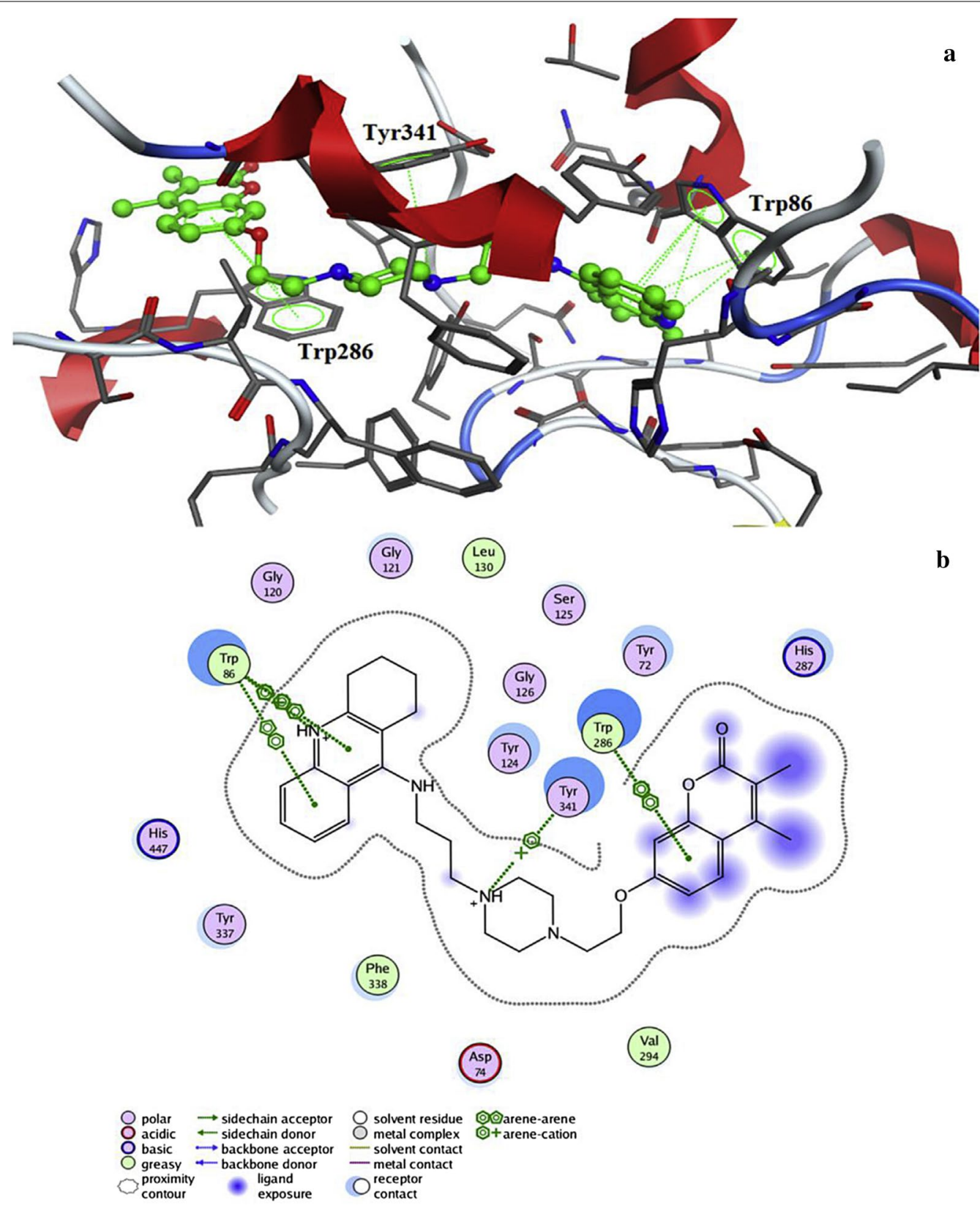

Fig. 85 a 3D docking model of compound C95 with AChE. b 2D schematic diagram of docking model of compound C95 with AChE [107]

precursor protein cleaving enzyme 1 (BACE1) enzyme activity. 2'-Isopropyl psoralene was isolated for the first time from Angelica decursiva. The results showed that among all the coumarins esculetin and daphnetin $(\mathbf{C} \mathbf{9 4} \mathbf{j})$ exhibited good AChE inhibitory activity with $\mathrm{IC}_{50}$ values of 6.13 and $11.57 \mu \mathrm{M}$ against berberine $\left(\mathrm{IC}_{50}=0.717 \mu \mathrm{M}\right)$, whereas daphnetin, esculetin, and umbelliferone 6-carboxylic acid exhibited highest BuChE inhibitory activity with $\mathrm{IC}_{50}$ values of $8.66,9.29$, and $27.19 \mu \mathrm{M}$ against berberine $\left(\mathrm{IC}_{50}=7.01 \mu \mathrm{M}\right)$. Both, esculetin and daphnetin have a catechol group which was noted to be significant for enhancing activity against $\mathrm{AChE}$ and $\mathrm{BuChE}$ and the presence of sugar moiety (umbelliferone 6-carboxylic acid, umbelliferone, scopoletin, isoscopoletin, 20-isopropylpsoralene, $\mathrm{IC}_{50}$ values 104.12 , 145.19, 150.28, 153.77 and $173.89 \mu \mathrm{M}$, respectively) as well methoxylation (7-methoxy coumarin $\mathrm{IC}_{50}=186.47 \mu \mathrm{M}$ ) were the main cause to diminish the activity. Finally, umbelliferone 6-carboxylic acid, esculetin and daphnetin exhibited promising BACE1 inhibitory activity with $\mathrm{IC}_{50}$ 

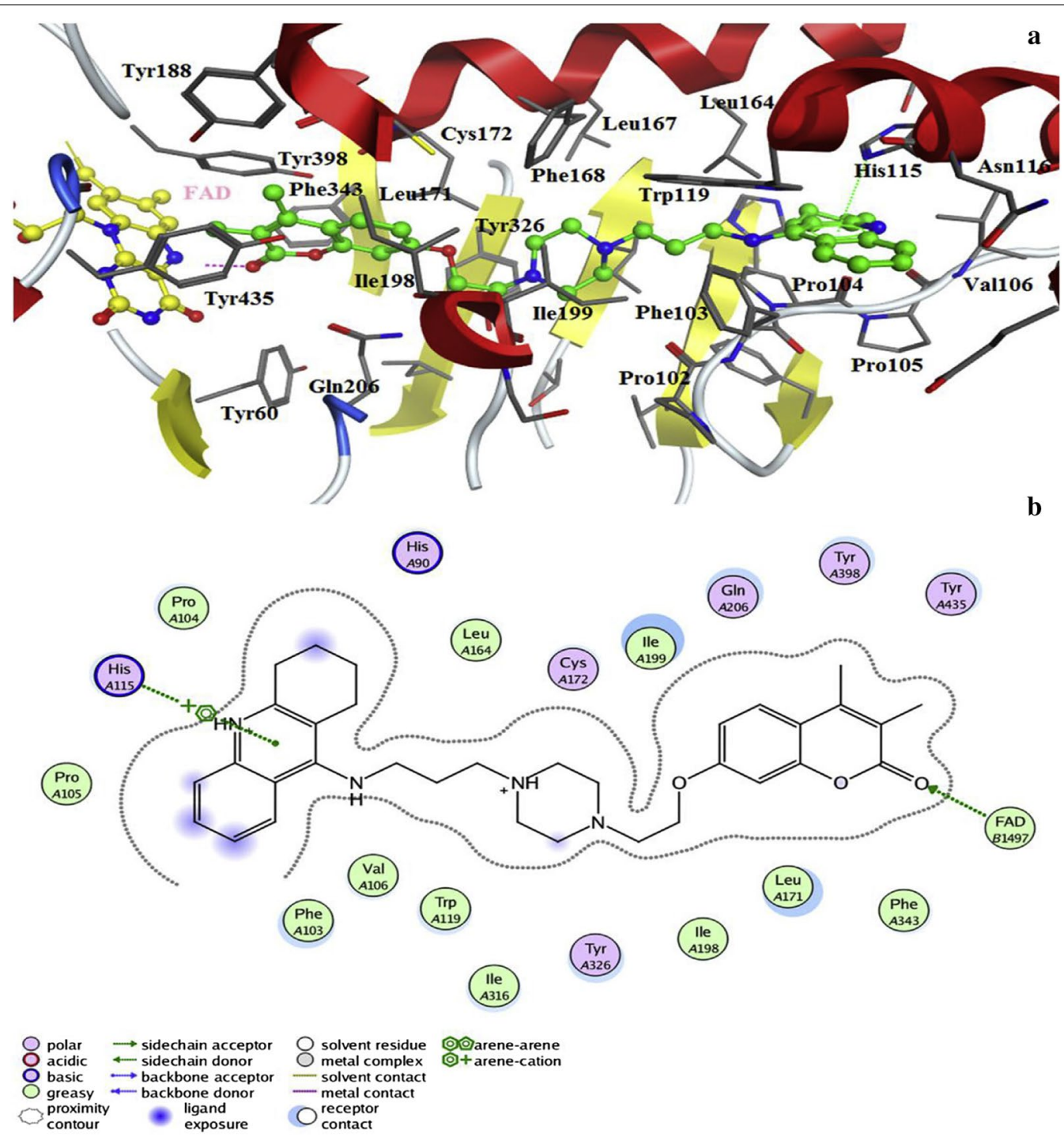

Fig. 86 (a) 3D docking model of compound C95 with MAO-B. b 2D schematic diagram of docking model of compound C95 with MAO-B [107]

values of $0.34,7.67$, and $11.19 \mu \mathrm{M}$ against quercetin $\left(\mathrm{IC}_{50}=13.98 \mu \mathrm{M}\right)$. These coumarins have carboxyl or catechol groups, which were reported to effectively enhance the anti-AD properties. They scrutinized the potentials of these three coumarins as BACE1 against the most potent nonpeptic BACE1 inhibitor reported to date according to protein data bank which is 2-amino-3-\{(1r)-1-cyclohexyl2-[(cyclohexylcarbonyl)amino] ethyl\}-6-phenoxyquinazolin-3-Ium (QUD). They generated a 3D structure of BACE1 by using Autodock 4.2. to perform the molecular docking studies. They found that the corresponding ligand interactions of umbelliferone 6-carboxylic acid in the active site of BACE1 was encouraged by hydrogenbonding interrelationship between the Arg235 residue and one carboxylic group interrelation between each of the Tyr71, Arg235 and Thr72 residues with one hydroxyl group at C-7 and one carboxylic group at C-6. Similarly, comparable ligand interaction for esculetin in the active site of BACE1, consisted of two hydrogen-bonding interactions with the Asn37, Ser36, and Ile126 residues of BACE1 and two hydroxyl groups of the compound, while binding of daphnetin in the active site of BACE1 was arbitrated by two hydrogen-bonding interrelations with Asp259 and Phe257 residues of the enzyme and two hydroxyl groups of the compound (Fig. 83). The binding energies of these three coumarin were $-4.58,-6.25$ and $-6.37 \mathrm{kcal} / \mathrm{mol}$, which was comparable to that of QUD $(-10.99 \mathrm{kcal} / \mathrm{mol})$. Their study concluded that additional hydrogen bonding and carboxylic groups could help in stabilizing the open form of BACE1 and could help in 


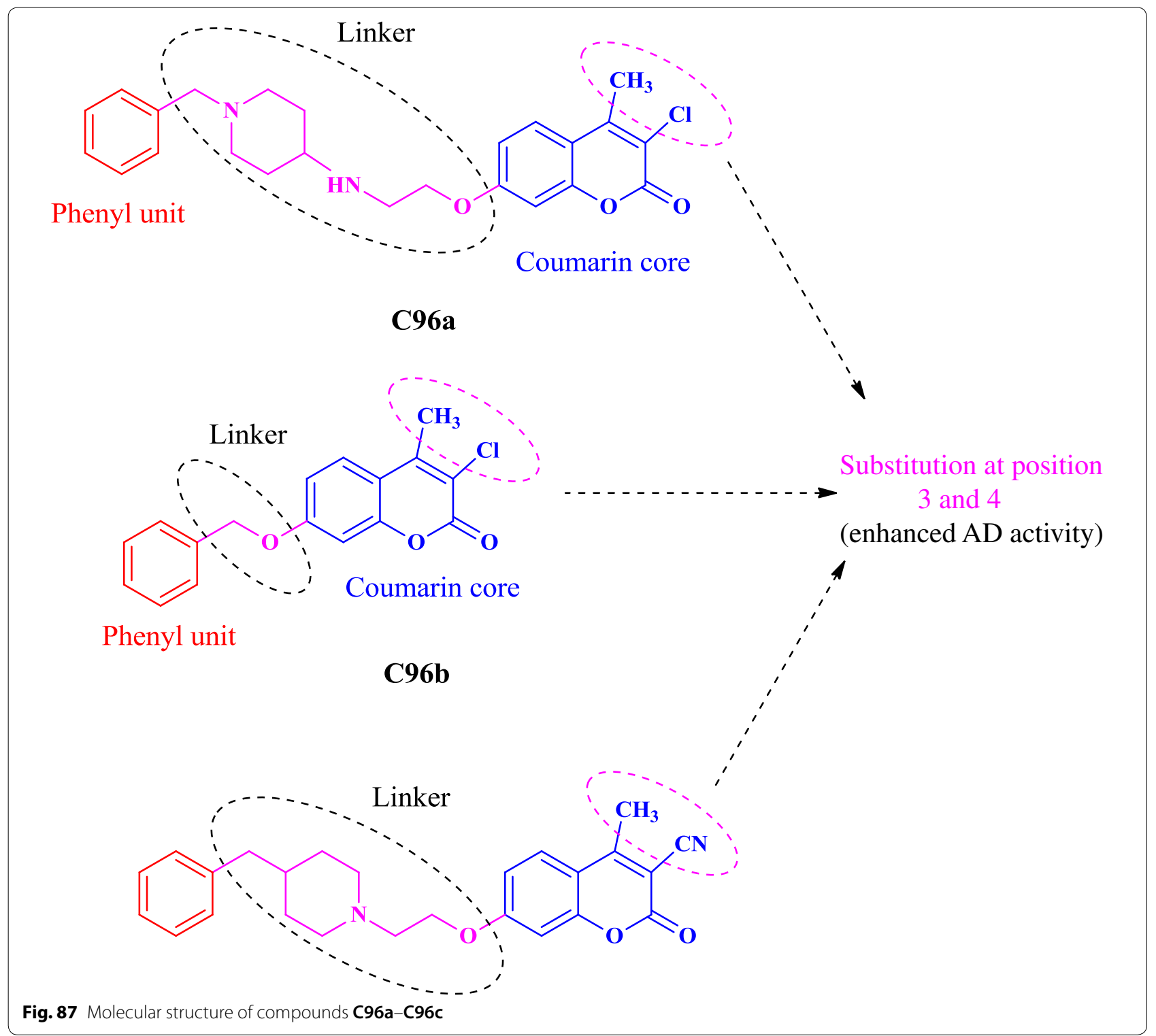

tighter binding to the active site, hence resulting in more effective enzyme inhibition [106].

In continuation to their previous work based on the synthesis of multi-target inhibitors of AD, Xie et al. [107] synthesized another series of multifunctional tacrinecoumarin hybrids and evaluated their performance as ChEs, MAOs and the ability to penetrate the blood-brain barrier (BBB). Most of the compounds displayed potent inhibitory activity towards AChE, BuChE but selective inhibition against MAO-B. The length of the linker bridging the coumarin nucleus and the tacrine unit was found to enhance the AChE activity whereas it didn't influenced much to the inhibition of BuChE and MAO-B. Among all the compounds, compound C95 was the most promising compound displaying highest inhibition for MAO-B, eelAChE, eqBuChE, hAChE as well as hBuChE and reported as the 'mixed type multifunctional strong inhibitor' for AD (Fig. 84). Its docking results revealed its simultaneous binding to CAS, PAS, mid-gorge site of $\mathrm{AChE}$ and covering the substrate and entrance cavities of MAO-B. Moreover, it also showed good penetration ability for the $\mathrm{BBB}$, regardless of the possible interaction with carriers or efflux transport proteins (Figs. 85 and 86) [107].

Joubert et al. [108] designed a series of 7-substituted coumarin ligands as multi-targeted inhibitors of $\mathrm{AD}$ and checked their ChEs bioactivity profile by application of Ellman's method using donepezil and tacrine 


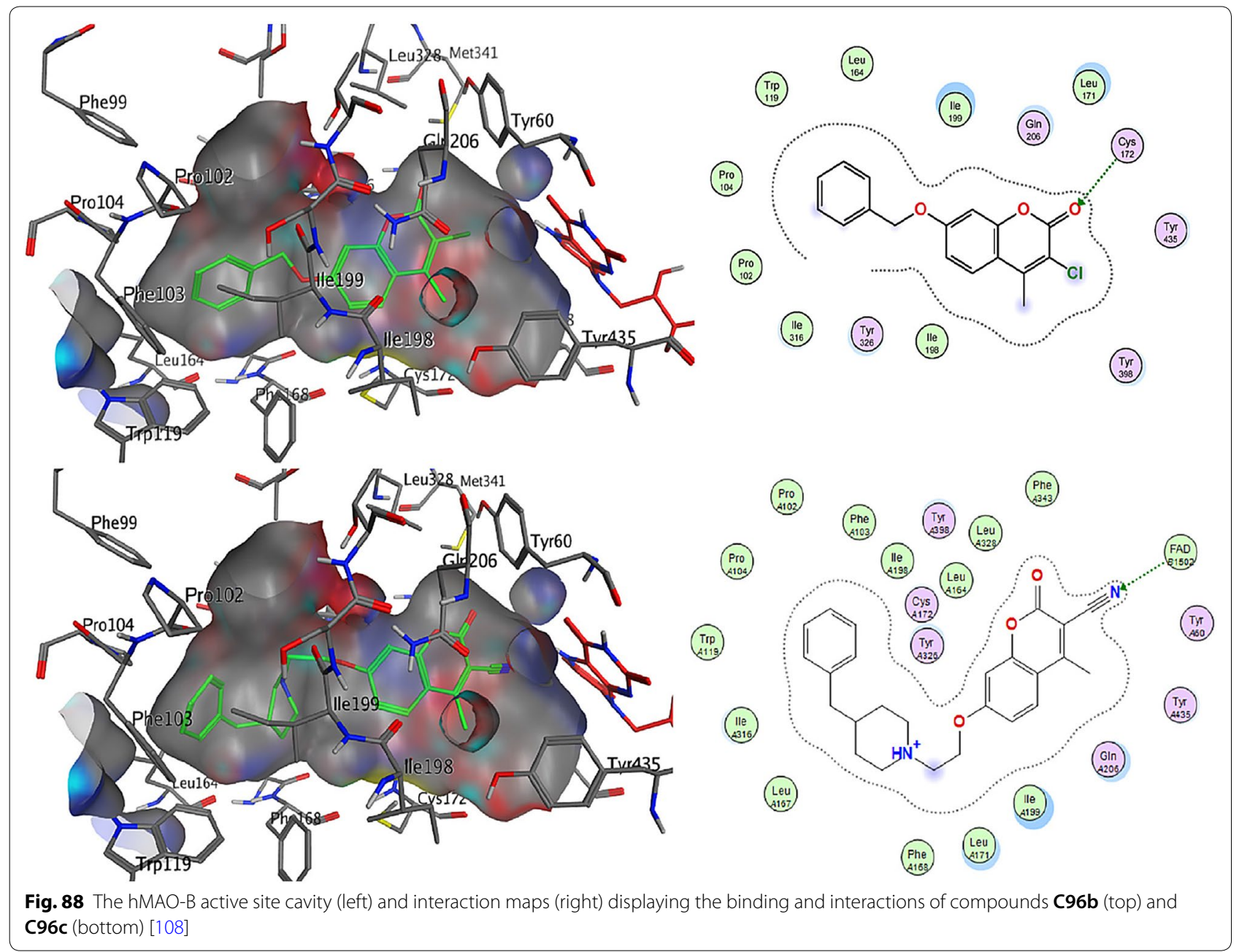

as standards against electrophorus electricus enzyme (eelAChE) and equine serum enzyme (eqBuChE). Most of the analogues exhibited potent inhibitory activity with $\mathrm{IC}_{50}$ values of micromolar and nanomolar. It is worth to mention that the alkyl ether linker at position-7 greatly influenced the inhibition. Compound C96a $\left(\mathrm{IC}_{50}=1.58 \mu \mathrm{M}\right)$ displayed highest AChE inhibitory and C96b $\left(\mathrm{IC}_{50}=0.96 \mu \mathrm{M}\right)$ displayed highest BuChE inhibitory power (Fig. 87). Interestingly all the synthesized compounds displayed considerable and selective MAO-B inhibitory activities when compared to the standards selegiline and clorgyline in the nanomolar range. Overall compound C96c was found to be the most important multifunctional ligand displaying balanced bioactivity profile to inhibit ChEs (eelAChE: $\mathrm{IC}_{50}=9.10 \mu \mathrm{M}$; eqBuChE: $\left.\mathrm{IC}_{50}=5.90 \mu \mathrm{M}\right)$ and hMAO-B $\left(\mathrm{IC}_{50}=0.30 \mu \mathrm{M}\right)$ along with C96b. Molecular docking was performed to get the insight of the binding modes and interaction of these two best-ranked compounds. The results obtained suggested that within the active site of MAO-B the coumarin moiety of both the compounds was bounded in the polar region of the substrate cavity, in the close vicinity of the FAD cofactor, and the 'aromatic sandwich' was defined by the residues Tyr398 and Tyr435. The carbonyl moiety of C96b showed a hydrogen bond interaction with Cys172 and the nitrile on the position-3 of C96c exhibited a hydrogen bond interaction with N5 of FAD. Moreover the benzyl- and $N$-benzylpiperidine side chain of C96b and C96c was found to extend and past the Ile199 which was situated in the entrance cavity of the enzyme. Within the hydrophobic environment of the entrance cavity, the benzyl- and $N$-benzylpiperidine side chain were reported to be stabilized by Van der Waals interactions. The binding orientations and interactions are depicted in Fig. 88. Moreover, the reason behind the selectivity for BuChE over $\mathrm{AChE}$ in case of compound C86b was reported to be mainly its inability to reach the CAS and having no interactions with its surrounding 

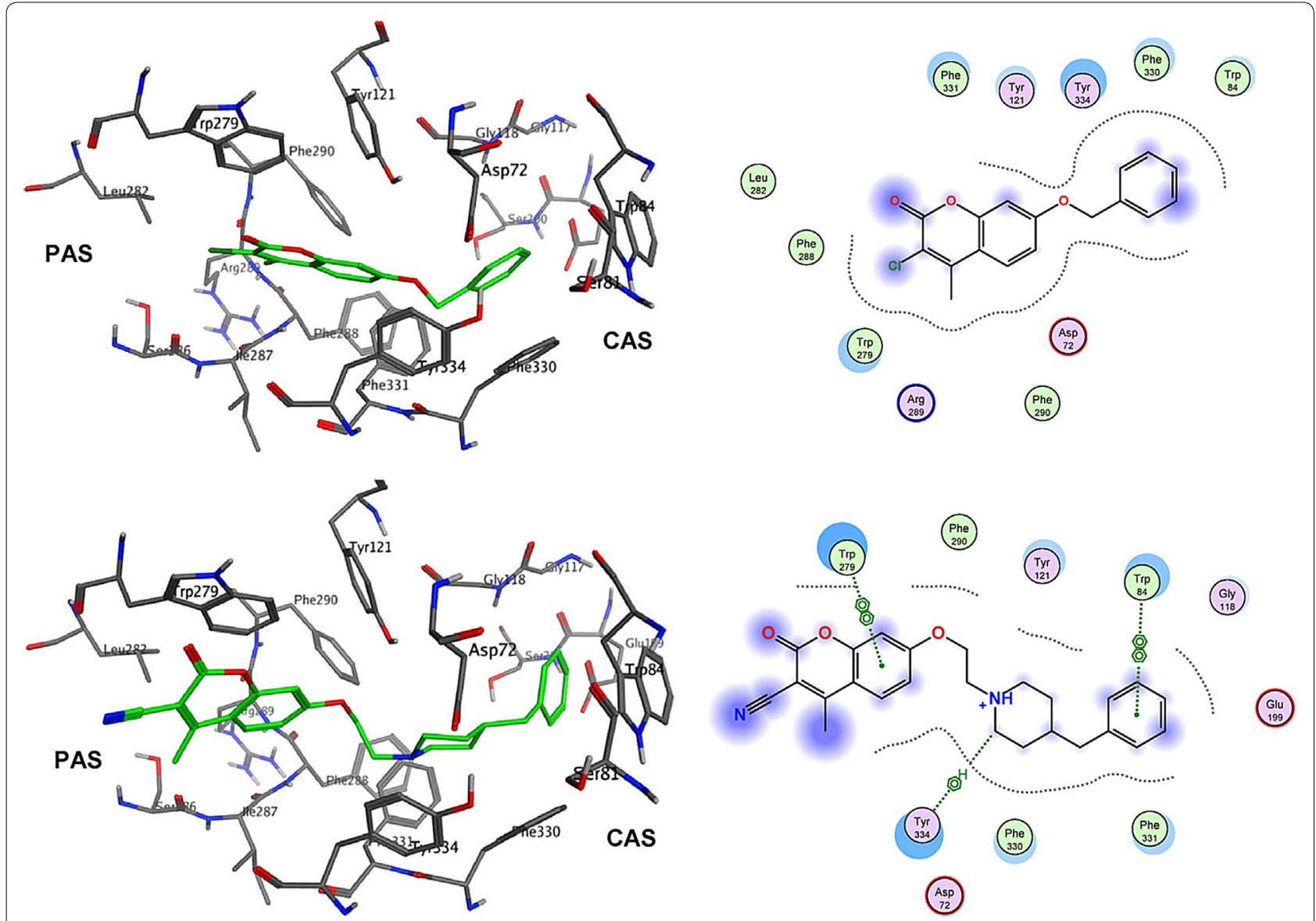

Fig. 89 The eelAChE active site cavity (left) and interaction maps (right) displaying the binding and interactions of compounds C96b (top) and C96c (bottom) [108]

residues. However, the results obtained for $\mathbf{C 9 6}$ showed that the coumarin moiety was bounded to the PAS site of the enzyme, establishing a $\pi-\pi$ stacking interaction between its phenyl ring and the indole ring of Trp279. Its piperidine moiety was found to interact with Tyr324 in the mid-gorge and its $N$-benzylpiperidine moiety which was located at the CAS, showed a $\pi-\pi$ stacking interaction with Trp84 (Fig. 89). The important docking results of these compounds as a good BuChE inhibitor dipicts that the benzyl- and coumarin moieties were bounded in the CAS and PAS region of the enzyme respectively and hence establishing a $\pi-\pi$ stacking interactions with Trp82 and Tyr332. C96c was found to be well accommodated within the active site gorge and showed a binding mode with a U-shaped conformation. The lowest energy binding orientation of C96c enabled its benzyl moiety to interact with Trp82 in the CAS allowing a $\pi-\pi$ stacking interaction (Fig. 90) [108].

\section{Conclusion}

$\mathrm{AD}$ remains among one of the serious problem and the most common cause of dementia worldwide. It causes depression, abnormal behaviour, anxiety as well as irreversible decline in cognitive, social and physical function. The present review presents coumarin and its derivatives as an important starting tool in the development of anti-Alzheimer drug discovery process. In search of new anti-Alzheimer drugs, coumarin-bearing derivatives have been synthesized abundantly and screened for their antiAlzheimer properties. Interestingly, many of these derivatives are found to possess promising activity due to their unique features that render them attractive for the discovery and development of novel anti-Alzheimer drugs.

Coumarins bearing acetamide pendant (C2) were reported as potent $\mathrm{AChE}$ inhibitors $\left(\mathrm{IC}_{50} 1.2 \mu \mathrm{M}\right)$ whereas coumarins with phenylpiperazine fragment (C8) were also found to be effective AChE inhibitors as they effectively adopt a sandwich structure by entering into the gorge of AChE enzyme, resulting in a parallel $\pi-\pi$ stacking. Insertion of aromatic groups at position 7 , and 


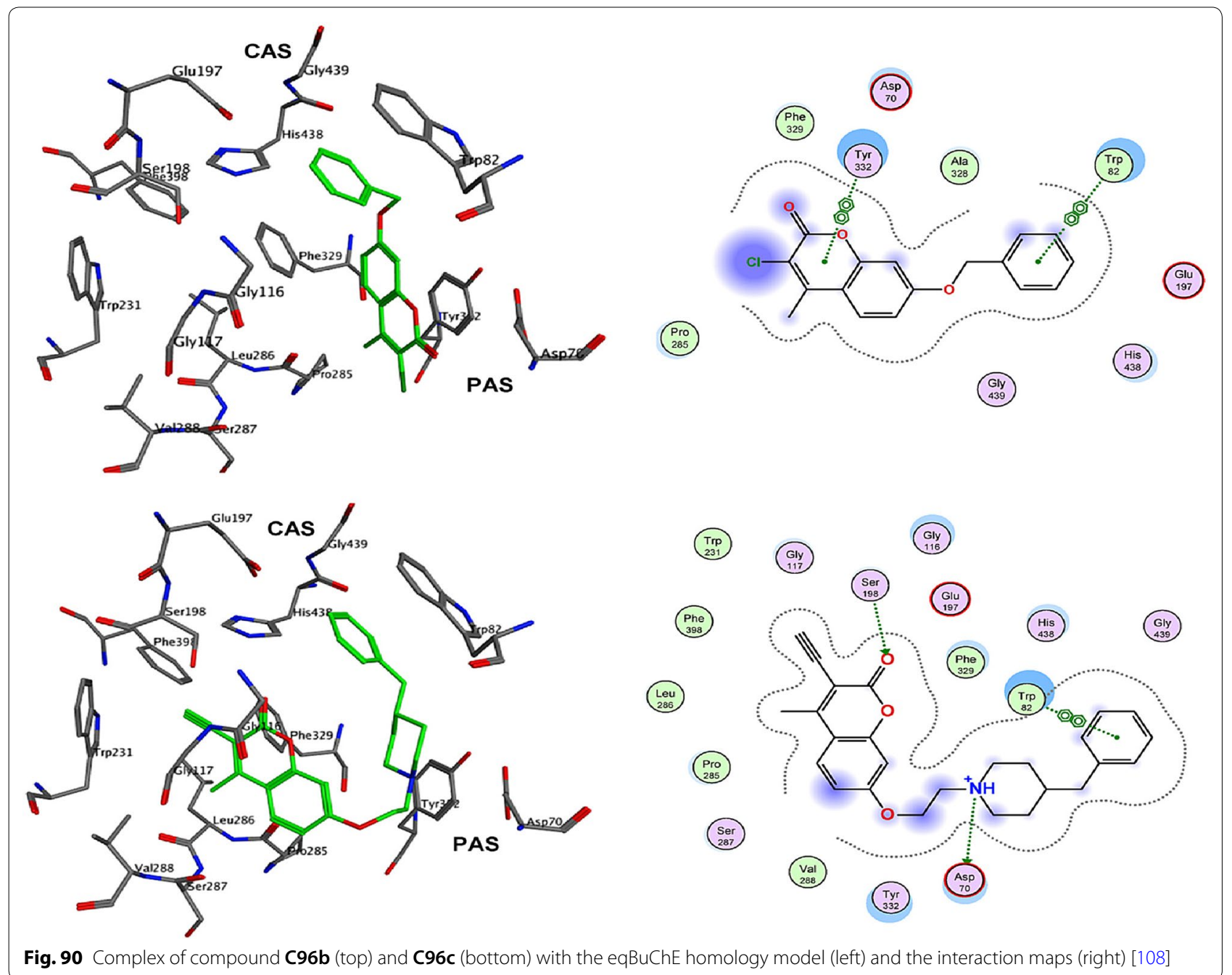

the presence of electron withdrawing substituents (for example fluoro) at position 4 in the coumarin core (C11) was found to effectively increase their dual ability to inhibit $\mathrm{AChE}$ and $\mathrm{BuChE}$. It is worth to mention the position of methoxy group, as it highly influences BuChE/ AChE selectivity in 71 versus 9000 ratio if it is inserted at positions 7- $(\mathbf{C 1 3 a})$ and 8- $(\mathbf{C 1 4 a})$ in the coumarin nucleus. Nevertheless, 3-acyl chlorides coumarin derivatives, such as $\mathbf{C 5 2}$ was reported to be active, against both the isoforms of MAO viz. MAO-A and MAO-B and in particular towards MAO-B with $\mathrm{IC}_{50}$ value of 8.0. Results has also revealed that if phenyl-imino group is present in the coumarin scaffold then it establishes favourable $\pi-\pi$ stacking interaction with the side chain of Phe108 of flap pocket as found for the cases $\mathbf{C 8 9 b}$ and C90b. Moreover, it was also reported that tacrine-coumarin hybrids act as multifunctional ChE inhibitors for AD treatment as reported for the compound $\mathbf{C 9 2}$.
In conclusion, further modifications in the coumarin nucleus could help in the development of new potential therapeutic agents with improved chemotherapeutic profiles, multifunctional role, reduced toxicity, short therapy time and rapid mechanism of action.

\section{Abbreviations \\ AD: Alzheimer's disease; NFTs: neurofibrillary tangles; Aß: beta-amyloid; APP: amyloid precursor protein; CSF: cerebrospinal fluid; AChE: acetylcholinesterase: MAO: monoamine oxidase; BuChE: butyrylcholinesterase; hAChE: human ace- tylcholinesterase; hBuChE: human butyrylcholinesterase; ROS: reactive oxygen species; BACE1: $\beta$-secretase; PAS: peripheral anionic site; eelAChE: electropho- rus electricus acetylcholinesterase; eqBuChE: horse serum butyrylcholinester- ase; TCAChE: Torpedo californica acetylcholinesterase; CAS: catalytic active site; ES: ecstatic site; ABS: acyl binding site; CS: catalytic site; FAD: flavin adenine dinucleotide: MPTP: 1-methyl-4-phenyl-1,2,3,6-tetrahydropyridine: MAOls: MAO-A and MAO-B inhibitors; ANS: 1-anilinonaphthalene-8-sulfonic acid.}

\section{Authors' contributions}

All authors read and approved the final manuscript. 


\begin{abstract}
Author details
1 School of Industrial Technology, Universiti Sains Malaysia, 11800 Penang, Malaysia. ${ }^{2}$ Industrial Chemistry Programme, Faculty of Science and Natural Resources, Universiti Malaysia Sabah, 88400 Kota Kinabalu, Sabah, Malaysia. ${ }^{3}$ School of Chemical Sciences, Universiti Sains Malaysia, 11800 Penang, Malaysia.
\end{abstract}

\section{Competing interests}

The authors declare that they have no competing interests.

\section{Funding}

SKY wish to express the gratitude and appreciation to School of Industrial Technology, Universiti Sains Malaysia, Penang and MSK thanks the Faculty of Science and Natural Resources, Universiti Malaysia Sabah (UMS), Kota Kinabalu for supporting this work with research Grant SLB0052-SG-2013.

\section{Publisher's Note}

Springer Nature remains neutral with regard to jurisdictional claims in published maps and institutional affiliations.

Received: 26 July 2018 Accepted: 21 November 2018

Published online: 04 December 2018

\section{References}

1. Salomone S, Caraci F, Leggio GM, Fedotova J, Drago F (2012) New pharmacological strategies for treatment of Alzheimer's disease: focus on disease modifying drugs. Br J Clin Pharmacol 73(4):504-517

2. Hampel $H$, Shen $Y$, Walsh DM, Aisen P, Shaw LM, Zetterberg $H$, Trojanowski JQ, Blennow K (2010) Biological markers of amyloid $\beta$-related mechanisms in Alzheimer's disease. Exp Neurol 223(2):334-346

3. Galimberti D, Ghezzi L, Scarpini E (2013) Immunotherapy against amyloid pathology in Alzheimer's disease. J Neurol Sci 333(1):50-54

4. Jack JCR, Knopman DS, Jagust WJ, Shaw LM, Aisen PS, Weiner MW, Petersen RC, Trojanowski JQ (2010) Hypothetical model of dynamic biomarkers of the Alzheimer's pathological cascade. Lancet Neurol 9(1):119-128

5. Schelterns P, Feldman H (2003) Treatment of Alzheimer's disease; current status and new perspectives. Lancet Neurol 2(9):539-547

6. Anand P, Singh B, Singh N (2012) A review on coumarins as acetylcholinesterase inhibitors for Alzheimer's disease. Bioorg Med Chem 20(3):1175-1180

7. Fallarero A, Oinonen P, Gupta S, Blom P, Galkin A, Mohan CG, Vuorela PM (2008) Inhibition of acetylcholinesterase by coumarins: the case of coumarin 106. Pharmacol Res 58(3-4):215-221

8. Khoobi M, Alipour M, Moradi A, Sakhteman A, Nadri H, Razavi SF, Ghandi M, Foroumadi A, Shafiee A (2013) Design, synthesis, docking study and biological evaluation of some novel tetrahydrochromeno [3', $\left.4^{\prime}: 5,6\right]$ pyrano $[2,3-b]$ quinolin-6 $(7 \mathrm{H})$-one derivatives against acetyland butyrylcholinesterase. Eur J Med Chem 68:291-300

9. Xie SS, Wang XB, Li JY, Yang L, Kong LY (2013) Design, synthesis and evaluation of novel tacrine-coumarin hybrids as multifunctional cholinesterase inhibitors against Alzheimer's disease. Eur J Med Chem 64:540-553

10. Asadipour A, Alipour M, Jafari M, Khoobi M, Emami S, Nadri H, Sakhteman A, Moradi A, Sheibani V, Moghadam FH, Shafiee A (2013) Novel coumarin-3-carboxamides bearing N-benzylpiperidine moiety as potent acetylcholinesterase inhibitors. Eur J Med Chem 70:623-630

11. Nebbioso M, Pascarella A, Cavallotti C, Pescosolido N (2012) Monoamine oxidase enzymes and oxidative stress in the rat optic nerve: age-related changes. Inte J Clin Exp Pathol 93(6):401-405

12. Huang M, Xie SS, Jiang N, Lan JS, Kong LY, Wang XB (2015) Multifunctional coumarin derivatives: monoamine oxidase $B(M A O-B)$ inhibition, anti- $\beta$-amyloid $(A \beta)$ aggregation and metal chelation properties against Alzheimer's disease. Bioorg Med Chem Lett 25(3):508-513

13. Youdim MB, Bakhle YS (2006) Monoamine oxidase: isoforms and inhibitors in Parkinson's disease and depressive illness. Br J Pharmacol 147(S1):287-296
14. Zatta P, Drago D, Bolognin S, Sensi SL (2009) Alzheimer's disease, metal ions and metal homeostatic therapy. Trends Pharmacol Sci 30(7):346-355

15. Piazzi L, Cavalli A, Colizzi F, Belluti F, Bartolini M, Mancini F, Recanatini M, Andrisano V, Rampa A (2008) Multi-target-directed coumarin derivatives: hAChE and BACE1 inhibitors as potential anti-Alzheimer compounds. Bioorg Med Chem Lett 18(1):423-426

16. Sashidhara KV, Kumar A, Chatterjee M, Rao KB, Singh S, Verma AK, Palit G (2011) Discovery and synthesis of novel 3-phenylcoumarin derivatives as antidepressant agents. Bioorg Med Chem Lett 21(7):1937-1941

17. Kostova I, Bhatia S, Grigorov P, Balkansky S, S Parmar V, K Prasad A, Saso L (2011) Coumarins as antioxidants. Curr Med Chem 18(25):3929-3951

18. Sashidhara KV, Kumar A, Kumar M, Sarkar J, Sinha S (2010) Synthesis and in vitro evaluation of novel coumarin-chalcone hybrids as potential anticancer agents. Bioorg Med Chem Lett 20(24):7205-7211

19. Kawaii S, Tomono Y, Ogawa K, Sugiura M, Yano M, Yoshizawa Y, Ito C, Furukawa $H$ (2001) Antiproliferative effect of isopentenylated coumarins on several cancer cell lines. Anticancer Res 21(3B):1905-1911

20. Barros TD, De Freitas LA, Filho JM, Nunes XP, Giulietti AM, De Souza GE, Dos Santos RR, Soares MB, Villarreal CF (2010) Antinociceptive and antiinflammatory properties of 7-hydroxycoumarin in experimental animal models: potential therapeutic for the control of inflammatory chronic pain. J Pharm Pharmacol 62:205-213

21. KhanYusufzai S, Osman H, Khan MS, Mohamad S, Sulaiman O, Parumasivam T, Gansau JA, Johansah N (2017) Design, characterization, in vitro antibacterial, antitubercular evaluation and structure-activity relationships of new hydrazinyl thiazolyl coumarin derivatives. Med Chem Res 26(6):1139-1148

22. Vasconcelos JF, Teixeira MM, Barbosa-Filho JM, Agra MF, Nunes XP, Giulietti AM, Ribeiro-dos-Santos R, Soares MB (2009) Effects of umbelliferone in a murine model of allergic airway inflammation. Eur J Pharmacol 609(1-3):126-131

23. Changwong N, Sabphon C, Ingkaninan K, Sawasdee P (2012) Acetyland butyryl-cholinesterase inhibitory activities of mansorins and mansonones. Phytother Res 26(3):392-396

24. Brühlmann C, Ooms F, Carrupt PA, Testa B, Catto M, Leonetti F, Altomare C, Carotti A (2001) Coumarins derivatives as dual inhibitors of acetylcholinesterase and monoamine oxidase. J Med Chem 44(19):3195-3198

25. Shen Q, Peng Q, Shao J, Liu X, Huang Z, Pu X, Ma L, Li YM, Chan AS, Gu L (2005) Synthesis and biological evaluation of functionalized coumarins as acetylcholinesterase inhibitors. Eur J Med Chem 40(12):1307-1315

26. Jin P, Kim JA, Choi DY, Lee YJ, Jung HS, Hong JT (2013) Anti-inflammatory and anti-amyloidogenic effects of a small molecule, 2, 4-bis ( $p$-hydroxyphenyl)-2-butenal in Tg2576 Alzheimer's disease mice model. J Neuroinflammation 10(1):767-779

27. Razavi SF, Khoobi M, Nadri H, Sakhteman A, Moradi A, Emami S, Foroumadi A, Shafiee A (2013) Synthesis and evaluation of 4-substituted coumarins as novel acetylcholinesterase inhibitors. Eur J Med Chem 64:252-259

28. Kapkova P, Stiefl N, Surig U, Engels B, Baumann K, Holzgrabe U (2003) Synthesis, biological activity, and docking studies of new acetylcholinesterase inhibitors of the bispyridinium type. Arch Pharm 336(11):523-540

29. Nam SO, Park DH, Lee YH, Ryu JH, Lee YS (2014) Synthesis of aminoalkylsubstituted coumarin derivatives as acetylcholinesterase inhibitors. Bioorg Med Chem 22(4):1262-1267

30. Singla S, Piplani $P$ (2016) Coumarin derivatives as potential inhibitors of acetylcholinesterase: synthesis, molecular docking and biological studies. Bioorg Med Chem 24(19):4587-4599

31. Zhou X, Wang XB, Wang T, Kong LY (2008) Design, synthesis, and acetylcholinesterase inhibitory activity of novel coumarin analogues. Bioorg Med Chem 16(17):8011-8021

32. Giacobini $E$ (2003) Cholinesterases: new roles in brain function and in Alzheimer's disease. Neurochem Res 28(3-4):515-522

33. Castro A, Martinez A (2001) Peripheral and dual binding site acetylcholinesterase inhibitors: implications in treatment of Alzheimer's disease. Mini Rev Med Chem 1(3):267-272

34. Weinstock M (1999) Selectivity of cholinesterase inhibition. CNS Drugs 12(4):307-323 
35. Hoerr R, Noeldner M (2002) Ensaculin (KA-672. HCl): a multitransmitter approach to dementia treatment. CNS Drug Rev 8(2):143-158

36. Sussman JL, Harel M (1991) Atomic structure of acetylcholinesterase from Torpedo californica: a prototypic acetylcholine-binding protein. Science 253(5022):872-879

37. Harel M, Quinn DM, Nair HK, Silman I, Sussman JL (1996) The X-ray structure of a transition state analog complex reveals the molecular origins of the catalytic power and substrate specificity of acetylcholinesterase. J Am Chem Soc 118(10):2340-2346

38. Radic Z, Pickering NA, Vellom DC, Camp S, Taylor P (1993) Three distinct domains in the cholinesterase molecule confer selectivity for acetyland butyrylcholinesterase inhibitors. Biochemistry 45:12074-12084

39. Taylor JL, Mayer RT, Himel CM (1994) Conformers of acetylcholinesterase: a mechanism of allosteric control. Mol Pharmacol 45(1):74-83

40. Pang YP, Quiram P, Jelacic T, Hong F, Brimijoin S (1996) Highly potent, selective, and low cost bis-tetrahydroaminacrine inhibitors of acetylcholinesterase steps toward novel drugs for treating Alzheimer's disease. J Biol Chem 271(39):23646-23649

41. Quinn DM (1987) Acetylcholinesterase: enzyme structure, reaction dynamics, and virtual transition states. Chem Rev 87(5):955-979

42. Rampa A, Bisi A, Valenti P, Recanatini M, Cavalli A, Andrisano V, Cavrini V, Fin L, Buriani A, Giusti P (1998) Acetylcholinesterase inhibitors: synthesis and structure-activity relationships of $\omega$-[N-Methyl- $N$-(3alkylcarbamoyloxyphenyl)-methyl] aminoalkoxyheteroaryl derivatives. J Med Chem 41(21):3976-3986

43. Decker M, Kraus B, Heilmann J (2008) Design, synthesis and pharmacological evaluation of hybrid molecules out of quinazolinimines and lipoic acid lead to highly potent and selective butyrylcholinesterase inhibitors with antioxidant properties. Bioorg Med Chem 16(8):4252-4261

44. Kamal MA, Klein P, Luo W, Li Y, Holloway HW, Tweedie D, Greig NH (2008) Kinetics of human serum butyrylcholinesterase inhibition by a novel experimental Alzheimer therapeutic, dihydrobenzodioxepine cymserine. Neurochem Res 33(5):745-753

45. Alipour M, Khoobi M, Foroumadi A, Nadri H, Moradi A, Sakhteman A, Ghandi M, Shafiee A (2012) Novel coumarin derivatives bearing $\mathrm{N}$-benzyl pyridinium moiety: potent and dual binding site acetylcholinesterase inhibitors. Bioorg Med Chem 20(24):7214-7222

46. Khoobi M, Alipour M, Sakhteman A, Nadri H, Moradi A, Ghandi M, Emami S, Foroumadi A, Shafiee A (2013) Design, synthesis, biological evaluation and docking study of 5-oxo-4, 5-dihydropyrano [3, 2-c] chromene derivatives as acetylcholinesterase and butyrylcholinesterase inhibitors. Eur J Med Chem 68:260-269

47. Alipour M, Khoobi M, Moradi A, Nadri H, Moghadam FH, Emami S, Hasanpour Z, Foroumadi A, Shafiee A (2014) Synthesis and anti-cholinesterase activity of new 7-hydroxycoumarin derivatives. Eur J Med Chem 82:536-544

48. Saeed A, Zaib S, Ashraf S, Iftikhar J, Muddassar M, Zhang KY, lqbal J (2015) Synthesis, cholinesterase inhibition and molecular modelling studies of coumarin linked thiourea derivatives. Bioorg Chem 63:58-63

49. Shaik JB, Palaka BK, Penumala M, Kotapati KV, Devineni SR, Eadlapalli S, Darla MM, Ampasala DR, Vadde R, Amooru GD (2016) Synthesis, pharmacological assessment, molecular modeling and in silico studies of fused tricyclic coumarin derivatives as a new family of multifunctional anti-Alzheimer agents. Eur J Med Chem 107:219-232

50. Alipour M, Khoobi M, Nadri H, Sakhteman A, Moradi A, Ghandi M, Foroumadi A, Shafiee A (2013) Synthesis of some new 3-coumaranone and coumarin derivatives as dual inhibitors of acetyl-and butyrylcholinesterase. Arch Pharm 346(8):577-587

51. Catto M, Pisani L, Leonetti F, Nicolotti O, Pesce P, Stefanachi A, Cellamare S, Carotti A (2013) Design, synthesis and biological evaluation of coumarin alkylamines as potent and selective dual binding site inhibitors of acetylcholinesterase. Bioorg Med Chem 21(1):146-152

52. Pisani L, Catto M, Giangreco I, Leonetti F, Nicolotti O, Stefanachi A, Cellamare S, Carotti A (2010) Design, synthesis, and biological evaluation of coumarin derivatives tethered to an edrophonium-like fragment as highly potent and selective dual binding site acetylcholinesterase inhibitors. Chem Med Chem 5(9):1616-1630

53. Ghanei-Nasab S, Khoobi M, Hadizadeh F, Marjani A, Moradi A, Nadri H, Emami S, Foroumadi A, Shafiee A (2016) Synthesis and anticholinesterase activity of coumarin-3-carboxamides bearing tryptamine moiety. Eur J Med Chem 121:40-46

54. Hamulakova S, Janovec L, Soukup O, Jun D, Kuca K (2017) Synthesis, in vitro acetylcholinesterase inhibitory activity and molecular docking of new acridine-coumarin hybrids. Int J Biol Macromol 104:333-338

55. Sonmez F, Zengin Kurt B, Gazioglu I, Basile L, Dag A, Cappello V, Ginex T, Kucukislamoglu M, Guccione S (2017) Design, synthesis and docking study of novel coumarin ligands as potential selective acetylcholinesterase inhibitors. J Enzyme Inhib Med Chem 32(1):285-297

56. Meng FC, Mao F, Shan WJ (2012) Design, synthesis, and evaluation of indanone derivatives as acetylcholinesterase inhibitors and metalchelating agents. Bioorg Med Chem Lett 22:4462-4466

57. Tasso B, Catto M, Nicolotti O (2011) Quinolizidinyl derivatives of bi- and tricyclic systems as potent inhibitors of acetyl and butyrylcholinesterase with potential in Alzheimer's disease. Eur J Med Chem 46:2170-2184

58. Yao D, Wang J, Wang G, Jiang Y, Shang L, Zhao Y, Huang J, Yang S, Wang J, Yu Y (2016) Design, synthesis and biological evaluation of coumarin derivatives as novel acetylcholinesterase inhibitors that attenuate $\mathrm{H} 2 \mathrm{O}$ 2-induced apoptosis in SH-SY5Y cells. Bioorg Chem 68:112-123

59. Greenawalt JW, Schnaitman C (1970) An appraisal of the use of monoamine oxidase as an enzyme marker for the outer membrane of rat liver mitochondria. J Cell Biol Mol Sci 46(1):173-179

60. Mondovì B (1985) Structure and functions of amine oxidases. CRC Press Inc, Boca Raton

61. Chiba K, Trevor A, Castagnoli JN (1984) Metabolism of the neurotoxic tertiary amine, MPTP, by brain monoamine oxidase. Biochem Biophys Res Commun 120(2):574-578

62. Fritz RR, Abell CW, Patel NT, Gessner W, Brossi A (1985) Metabolism of the neurotoxin in MPTP by human liver monoamine oxidase B. FEBS Lett 186(2):224-228

63. Grimsby J, Toth M, Chen K, Kumazawa T, Klaidman L, Adams JD, Karoum F, Gal J, Shih JC (1997) Increased stress response and $\beta$-phenylethylamine in MAOB-deficient mice. Nature Genetics 7(2):206-210

64. De Zutter GS, Davis RJ (2001) Pro-apoptotic gene expression mediated by the p38 mitogen-activated protein kinase signal transduction pathway. Proc Natl Acad Sci USA 98(11):6168-6173

65. Johnston JP (1968) Some observations upon a new inhibitor of monoamine oxidase in brain tissue. Biochem Pharmacol 17(7):1285-1297

66. Weyler W, Hsu YP, Breakafield XO (1990) Biochemistry and genetics of monoamine oxidase. Pharmacol Ther 47(3):391-417

67. Westlund KN, Denney RM, Kochersperger LM, Rose RM, Abell CW (1985) Distinct monoamine oxidase $A$ and $B$ populations in primate brain. Science 230(4722):181-183

68. Geha RM, Rebrin I, Chen K, Shih JC (2001) Substrate and inhibitor specificities for human monoamine oxidase A and B are influenced by a single amino acid. J Biol Chem 276(13):9877-9882

69. Anderson MC, Hasan F, McCrodden JM, Tipton KF (1993) Monoamine oxidase inhibitors and the cheese effect. Neurochem Res 18(11):1145-1149

70. Wouters J (1998) Structural aspects of monoamine oxidase and its reversible. Curr Med Chem 5(2):137-162

71. McMillan FH, Leonard F, Meltzer RI, King JA (1953) Antitubercular substances. II. Substitution products of isonicotinic hydrazide. J Am Pharm Assoc 42(8):457-464

72. Fowler CJ, Oreland L, Callingham BA (1981) The acetylenic monoamine oxidase inhibitors clorgyline, deprenyl, pargyline and J-508: their properties and applications. J Pharm Pharmacol 33(1):341-347

73. Moureau F, Wouters J, Vercauteren DP, Collin S, Evrard G, Durant F, Ducrey F, Koenig JJ, Jarreau FX (1992) A reversible monoamine oxidase inhibitor, toloxatone: structural and electronic properties. Eur J Med Chem 27(9):939-948

74. Moureau F, Wouters J, Vercauteren DP, Collin S, Evrard G, Durant F, Ducrey F, Koenig JJ, Jarreau FX (1994) A reversible monoamine oxidase inhibitor, Toloxatone: spectrophotometric and molecular orbital studies of the interaction with flavin adenine dinucleotide (FAD). Eur J Med Chem 29(4):269-277

75. Moureau F, Wouters J, Depas M, Vercauteren DP, Durant F, Ducrey F, Koenig JJ, Jarreau FX (1995) A reversible monoamine oxidase inhibitor, Toloxatone: comparison of its physicochemical properties with those of 
other inhibitors including Brofaromine, Harmine, R40519 and Moclobemide. Eur J Med Chem 30(11):823-837

76. Sorbera LA, Rabasseda X, Castaner J (1992) AVASIMIBE. Drugs Future 24(1):9-15

77. Wouters J, Moureau F, Evrard G, Koenig JJ, Jegham S, George P, Durant F (1999) A reversible monoamine oxidase A inhibitor, befloxatone: structural approach of its mechanism of action. Bioor Med Chem 7(8):1683-1693

78. Bergström M, Westerberg G, Nemeth G, Traut M, Gross G, Greger G, Müller-Peltzer H, Safer A, Eckernäs SÅ, Grahner A, Långström B (1997) MAO-A inhibition in brain after dosing with esuprone, moclobemide and placebo in healthy volunteers: in vivo studies with positron emission tomography. Eur J Clin Pharmacol 52(2):121-128

79. Löscher W, Lehmann H, Teschendorf HJ, Traut M, Gross G (1999) Inhibition of monoamine oxidase type $A$, but not type $B$, is an effective means of inducing anticonvulsant activity in the kindling model of epilepsy. J Pharmacol Exp Ther 288(3):984-992

80. Pacher P, Kohegyi E, Kecskemeti V, Furst S (2001) Current trends in the development of new antidepressants. Cur Med Chem 8(2):89-100

81. Pacher P, Kecskemeti V (2004) Trends in the development of new antidepressants. Is there a light at the end of the tunnel? Cur Med Chem 11(7):925-943

82. Carreiras MC, Marco JL (2004) Recent approaches to novel anti-Alzheimer therapy. Curr Pharm Des 10(25):3167-3175

83. Binda C, Newton-Vinson P, Hubálek F, Edmondson DE, Mattevi A (2002) Structure of human monoamine oxidase B: a drug target for the treatment of neurological disorders. Nat Struct Mol Biol 9(1):22-26

84. Binda C, Wang J, Pisani L, Caccia C, Carotti A, Salvati P, Edmondson DE, Mattevi A (2007) Structures of human monoamine oxidase B complexes with selective noncovalent inhibitors: safinamide and coumarin analogs. J Med Chem 50(23):5848-5852

85. Matos MJ, Viña D, Picciau C, Orallo F, Santana L, Uriarte E (2009) Synthesis and evaluation of 6-methyl-3-phenylcoumarins as potent and selective MAO-B inhibitors. Bioorg Med Chem Lett 19(17):5053-5055

86. Viña D, Matos MJ, Yáñez M, Santana L, Uriarte E (2012) 3-Substituted coumarins as dual inhibitors of AChE and MAO for the treatment of Alzheimer's disease. Med Chem Comm 3(2):213-218

87. Gnerre C, Catto M, Leonetti F, Weber P, Carrupt PA, Altomare C, Carotti A, Testa B (2000) Inhibition of monoamine oxidases by functionalized coumarin derivatives: biological activities, QSARs, and 3D-QSARs. J Med Chem 43(25):4747-4758

88. Chimenti F, Secci D, Bolasco A, Chimenti P, Granese A, Befani O, Turini P, Alcaro S, Ortuso F (2004) Inhibition of monoamine oxidases by coumarin-3-acyl derivatives: biological activity and computational study. Bioorg Med Chem Lett 14(14):3697-3703

89. Secci D, Carradori S, Bolasco A, Chimenti P, Yáñez M, Ortuso F, Alcaro S (2011) Synthesis and selective human monoamine oxidase inhibition of 3-carbonyl, 3-acyl, and 3-carboxyhydrazido coumarin derivatives. Eur J Med Chem 46(10):4846-4852

90. Matos MJ, Vazquez-Rodriguez S, Uriarte E, Santana L, Viña D (2011) MAO inhibitory activity modulation: 3-Phenylcoumarins versus 3-benzoylcoumarins. Bioorg Med Chem Lett 21(14):4224-4227

91. Abdelhafez OM, Amin KM, Ali HI, Abdalla MM, Batran RZ (2013) Monoamine oxidase $A$ and $B$ inhibiting effect and molecular modeling of some synthesized coumarin derivatives. Neurochem Int 62(2):198-209

92. He X, Chen YY, Shi JB, Tang WJ, Pan ZX, Dong ZQ, Song BA, Li J, Liu XH (2014) New coumarin derivatives: design, synthesis and use as inhibitors of hMAO. Bioorg Med Chem 22(14):3732-3738
93. Nayak BV, Ciftci-Yabanoglu S, Bhakat S, Timiri AK, Sinha BN, Ucar G, Soliman ME, Jayaprakash V (2015) Monoamine oxidase inhibitory activity of 2-aryl-4H-chromen-4-ones. Bioorg Chem 58:72-80

94. Matos MJ, Terán C, Pérez-Castillo Y, Uriarte E, Santana L, Viña D (2011) Synthesis and study of a series of 3-arylcoumarins as potent and selective monoamine oxidase B inhibitors. J Med Chem 54(20):7127-7137

95. Matos MJ, Rodríguez-Enríquez F, Vilar S, Santana L, Uriarte E, Hripcsak G, Estrada M, Rodríguez-Franco MI, Viña D (2015) Potent and selective MAO-B inhibitory activity: amino-versus nitro-3-arylcoumarin derivatives. Bioorg Med Chem Lett 25(3):642-648

96. Matos MJ, Vilar S, Gonzalez-Franco RM, Uriarte E, Santana L, Friedman C, Tatonetti NP, Viña D, Fontenla JA (2013) Novel (coumarin-3-yl) carbamates as selective MAO-B inhibitors: synthesis, in vitro and in vivo assays, theoretical evaluation of ADME properties and docking study, Eur J Med Chem 63:151-161

97. Serra S, Ferino G, Matos MJ, Vázquez-Rodríguez S, Delogu G, Viña D, Cadoni E, Santana L, Uriarte E (2012) Hydroxycoumarins as selective MAO-B inhibitors. Bioorg Med Chem Lett 22(1):258-261

98. Pisani L, Catto M, Nicolotti O, Grossi G, Di Braccio M, Soto-Otero R, Mendez-Alvarez E, Stefanachi A, Gadaleta D, Carotti A (2013) Fine molecular tuning at position 4 of $2 \mathrm{H}$-chromen-2-one derivatives in the search of potent and selective monoamine oxidase B inhibitors. Eur J Med Chem 70:723-739

99. Xie L, Takeuchi Y, Cosentino LM, Lee KH (1999) Anti-AIDS agents. 37. Synthesis and structure-activity relationships of ( $\left.3^{\prime \prime} \mathrm{R}, 4^{\prime \prime} \mathrm{R}\right)-(+)$-ciskhellactone derivatives as novel potent anti-HIV agents. J Med Chem 42(14):2662-2672

100. Raev LD, Voinova E, Ivanov IC, Popov D (1990) Antitumor activity of some coumarin derivatives. Pharmazie 45(9):696-699

101. Rahman FS, Yusufzai SK, Osman H, Mohamad D (2016) Synthesis, characterisation and cytotoxicity activity of thiazole substitution of coumarin derivatives (characterisation of coumarin derivatives). J Phys Sci 27(1):77-87

102. Holla BS, Malini KV, Rao BS, Sarojini BK, Kumari NS (2003) Synthesis of some new 2, 4-disubstituted thiazoles as possible antibacterial and anti-inflammatory agents. Eur J Med Chem 38(3):313-318

103. Kadir SL, Yaakob H, Zulkifli RM (2013) Potential anti-dengue medicinal plants: a review. J Nat Med 67(4):677-689

104. Ranade DS, Bapat AM, Ramteke SN, Joshi BN, Roussel P, Tomas A, Deschamps P, Kulkarni PP (2016) Thiosemicarbazone modification of 3-acetyl coumarin inhibits $A \beta$ peptide aggregation and protect against A -induced cytotoxicity. Eur J Med Chem 121:803-809

105. Edraki N, Firuzi O, Foroumadi A, Miri R, Madadkar-Sobhani A, Khoshneviszadeh M, Shafiee A (2013) Phenylimino-2H-chromen-3-carboxamide derivatives as novel small molecule inhibitors of $\beta$-secretase (BACE1). Bioorg Med Chem 21(8):2396-2412

106. Ali MY, Jannat S, Jung HA, Choi RJ, Roy A, Choi JS (2016) Anti-Alzheimer's disease potential of coumarins from Angelica decursiva and Artemisia capillaris and structure-activity analysis. Asian Pac J Trop Med 9(2):103-111

107. Xie SS, Wang X, Jiang N, Yu W, Wang KD, Lan JS, Li ZR, Kong LY (2015) Multi-target tacrine-coumarin hybrids: cholinesterase and monoamine oxidase B inhibition properties against Alzheimer's disease. Eur J Med Chem 95:153-165

108. Joubert A, Foka GB, Repsold BP, Oliver DW, Kapp E, Malan SF (2017) Synthesis and evaluation of 7-substituted coumarin derivatives as multimodal monoamine oxidase- $B$ and cholinesterase inhibitors for the treatment of Alzheimer's disease. Eur J Med Chem 125:853-864 